\title{
Probleme der Potentialtheorie
}

\section{Doctoral Thesis}

\section{Author(s):}

Bolliger, Adolf

Publication date:

1917

Permanent link:

https://doi.org/10.3929/ethz-a-000097695

Rights / license:

In Copyright - Non-Commercial Use Permitted 


\section{Probleme der Potentialtheorie}

Von der

Eidgenössischen Technischen Hochschule

in Zürich

zur Erlangung der

Würde eines Doktors der technischen Wissenschaften

genehmigte

Promotionsarbeit

vorgelegt von

\section{Adolf Bolliger}

dipl. Elektro-Ing.

aus Holziken (Aargau)

Referent: Herr Prof. Dr. H. Weyl

Korreferent: Herr Prof. Dr. A. Schweitzer

147.

1917

Verlagsbuchhandlung von Julius Springer in Berlin 
Sonder-Abdruck aus „Archiv für Elektrotechnik“. 


\section{Einleitung.}

Anläßlich einer empirischen Untersuchung, die ich vor etwas mehr als zwei Jahren für Herrn Prof. Dr: K. Kuhlmann auf dem Gebiete der Potentialtheorie vorzunehmen hatte, kam ich auf den Gedanken, das auf den Seiten 32 und 33 dieser Schrift behandelte „Isolatorproblem" rechnerisch zu verfolgen. Mit Herrn Prof. Dr. H. Weyl, der mir dazu in liebenswürdiger Weise seine Hilfe bot, entstanden so die Anfänge der vorliegenden Arbeit. Ich benutze die Gelegenheit, Herrn Prof. Dr. H. Weyl an dieser Stelle für seine Mühe meinen Dank auszusprechen. Die übrigen in der Schrift enthaltenen mathematischen Entwicklungen sind von mir zum größten Teile in den Monaten Februar bis Mai des Jahres I9I5 während meiner Assistententätigkeit bei Herrn Prof. Dr. M. Großmann selbständig durchgearbeitet worden.

Die „Formeltabelle“ gibt uns die wichtigsten analytischen Hilfsmittel - es sind dies das Fouriersche Integraltheorem und verwandte Theoreme - an die Hand für die mathematische Behandlung der elektrostatischen Probleme, deren physikalische Grundgleichungen man in kurzer Übersicht in Abschnitt I findet. Der darauffolgende Abschnitt II löst das Potentialproblem für eine willkürliche konzentrische Verteilung des P.otentiales an der "Kreislochscheibe“, worunter ich eine Ebene mit kreisrundem Ausschnitt verstehe. Die hierfür gültige allgemeine Lösung wird dann im nächsten $\mathrm{Ab}$ schnitt III auf einen konkreten Einzelfall, wo die Influenzwirkung eines elektrischen Poles auf die Kreislochscheibe untersucht wird, angewendet.

Im anschließenden Abschnitt IV wird die Theorie der linearen Leiter vervollständigt. Es wird dort gezeigt, wie die linearen Leiter mit ihren wesentlichen Eigenschaften in der richtigen Weise durch Grenzübergänge aus räumlichen Leitern gewonnen werden können. Einer beliebigen Permeabilität des Leitermaterials wird durch ein zusätzliches Elementargesetz, das dort formuliert wird, Rechnung getragen. Beiläufig wird auch das zur Berechnung von Selbstinduktionskoeffizienten nicht mehr geeignete Neumannsche Konfigurationsintegral durch eine für dünne Leiter sehr exakte asymptotische Darstellung ersetzt, welche auch die Leiterdicke berücksichtigt. Zudem wird die Massenverteilung am linearen Leiter für das den Strömungszustand im Leiter beeinflussende elektrische Potential, worüber in der Literatur der linearen Leiter die Angaben durchweg fehlen, bestimmt.

In Abschnitt $\mathrm{V}$ werden die allgemeinen Integrale des induzierten elektrischen Feldes von zeitlich veränderlichen linearen Strömen zur Berechnung der iṇduzierten Spannung des Kreisleiters und des diesbezüglichen asymptotischen Selbstinduktionskoeffizienten verwendet und in Abschnitt VI finden sich verschiedene Anwendungsbeispiele für das elektrische (statische) Potential von linearen Leitern. So wird dort unter A das „Stab-Ring-Potential“ (Potential eines. linearen Stäbchens und eines damit kombinierten Drahtrings) und unter B das „Freileitungspotential“ ermittelt. Das Freileitungspotential ist die Idealisierung des bei Freileitungen vorkommenden elektrischen Potentiales. Die dafür gegebene Lösung bezieht sich auf lineare Leiter allgemeinster Art und berücksichtigt willkürliche Spannungsabfälle. Für die numerische 
Berechnung werden zwei leicht diskutierbare Formeln angegeben, die das Freileitungspotential geradliniger, linearer Leiterstücke mit dem Ohmschen Gesetze entsprechenden linearen Spannungsabfällen darstellen und zufolge des Superpositionsprinzipes in der Anwendung auf ganze Freileitungen übertragen werden können.

Das Potentialproblem, das sich, auf das elektrostatische System, bestehend aus einer Kreislochscheibe und einem linearen Leiter in der Achse derselben bezieht, wurde in der Schrift als "Isolatorproblem“ bezeichnet. Das erwähnte Randwertsystem entspricht dem Idealfälle des bei Hochspannungsdurchführungsisolatoren vorkommenden elektrischen Systemes. In Abschnitt VII werden dafür zwei Fälle unterschieden; nämlich wo der lineare Leiter unbegrenzt und wo derselbe von endlicher Länge ist. Im ersten Falle läßt, sich auf einfachem Wege die Potentialfunktion aus dem seiner mannigfachen Anwendungen wegen berïhmten Potentiale des Ellipsoides ableiten, und im źweiten Falle gewinnen wir die allgemeine Lösung für beliebige konzentrische Randwerte untér Zuhilfenahme des Superpositionsprinzipes aus der Kreislochscheibenlösung tund dem elektrostatischen "Potentiale der linearen Leiter. Im darauffolgenden $\mathrm{Ab}$ schnitt VII wird mittels des Fourierschen Integraltheoremes bei gegebener axialer Verteilung das Potentialproblem für einen unendlich langen Rotationszylinder gelöst und schließlich noch im letzten Abschnitt IX der Schrift darüber eine interessante Anwendung bei der Berechnung des „Zylinderhohlraumpotentiales" gemacht. In diesem Falle befindet sich irgendwo in der Achise eines hohlen, geerdeten zylindrischen Leiters ein elektrischer Massenpol. Das Zylinderhohlraumpotential kann aufgefaßt werden als Elementarpotential für den Isolătionsraum des einadrigen Kabels bei willkürlicher Verteilung längs der Kabelachse.

Zürich, Juli IgI6:

\section{Der Verfasser.}




\title{
Probleme der Potentialtheorie.
}

\author{
Inhaltsverzeichnis.
}

Einleitung ................ . . III

Wichtige analytische Hilfsmittel (Formeltabelle) ... . . . I

I. Die zur Lösung der Probleme verwendeten Grundgleichungen der Elektrostatik ............... 2

II. Kreislochscheibe . . . . . . . . . . . . . 5

III. Influenzwirkung eines elektrischen Poles auf die Kreislochscheibe..................... . . 8

IV. Vervollständigte Theorie der linearen Leiter für das Vakuum I3

V. Induzierte Spannung und asymptotischer Selbstinduktionskoeffizient des Kreisleiters . . . . . . . . . . . . . . 24

VI. Anwendungsbeispiele fur das elektrische Potential linearer Leiter:

A. Stab-Ring-Potential ............... 27

B. Freileitungspotential ............. 28

VII. Isolatorproblem . . . . . . . . . . . . . 32

VIII. Axiale Verteilung an einem unendlich langen Rotationszylinder 37

IX. Zylinder-Hohlraumpotential . . . . . . . . . . 40 Anhang ............... . . . 45 Curriculum vitae ................. 55

Wichtige analytische Hilfsmittel.

(Formeltabelle.)

I. Das Fouriersche Integraltheorem in den folgenden Darstellungen:
A. $\mathrm{f}(\mathrm{x})=\frac{2}{\pi} \int_{0}^{\infty} \sin (\kappa \mathrm{x}) \mathrm{d} \alpha \int_{0}^{\infty} \mathrm{f}(\lambda) \sin (\alpha \lambda) \mathrm{d} \lambda$
B. $f(x)=\frac{2}{\pi} \int_{0}^{\infty} \cos (\alpha x) d \alpha \int_{0}^{\infty} f(\lambda) \cos (\alpha \lambda) \mathrm{d} \lambda$
C. $f(x)=\frac{I}{\pi} \int_{0}^{\infty} \mathrm{d} \alpha \int_{-\infty}^{+\infty} \mathrm{f}(\lambda) \cos \alpha(\lambda-\mathrm{x}) \mathrm{d} \lambda$.

[Vergleiche daruber: Riemann-Weber, Partielle Differentialgleichungen Bd. I, 5. Aufl., S. 49,52 und 47 .]

II. Das dem Fourierschen Theorem analoge Integraltheorem der Besselschen Funktion nullter Ordnung $\mathrm{J}(\mathrm{x})$ :

$$
f(r)=\int_{0}^{\infty} J(\alpha \mathrm{r}) \propto \mathrm{d} \alpha \int_{0}^{\infty} \mathrm{f}(\lambda) \mathrm{J}(\alpha \lambda) \lambda \mathrm{d} \lambda
$$

[Riemann-Weber, Partielle Differentialgleichungen Bd. I, 5. Aufl., Seite 200.] Bolliger, Potentialtheorie. 
III. Die Integralrelation von M. Hafen in Wien:

$$
\frac{2}{\pi} \int_{a}^{\mathrm{x}} \frac{\eta \mathrm{d} \eta}{\sqrt{\mathrm{x}^{2}-\eta^{2}}} \int_{\mathrm{a}}^{\eta} \frac{\mathrm{f}(\xi)}{\sqrt{\eta^{2}-\xi^{2}}} \mathrm{~d} \xi=\int_{\mathrm{a}}^{\mathrm{x}} \mathrm{f}(\mathrm{x}) \mathrm{dx}
$$

[Mathematische Annalen Bd. 69, 1910, S. 517.]

IV. Das Theorem:

$$
\left.\mathrm{F}(\mathbf{r})=-\frac{2}{\pi} \int_{\mathrm{r}}^{\infty} \frac{\mathrm{d} \xi}{\sqrt{\xi^{2}-\mathrm{r}^{2}}} \cdot \frac{\mathrm{d} \int_{\xi}^{\infty} \frac{\mathrm{F}(\eta)}{\sqrt{\eta^{2}-\xi^{2}}} \eta \mathrm{d} \eta}{\mathrm{d} \xi}-{ }^{1}\right) .
$$

\section{Dịe zur Lösung der Probleme verwendeten Grundgleichungen der Elektrostatik.}

Schon von Lagrange ist erkannt worden, daß sich die Komponenten der von statischen Ladungen herrührenden elektrischen Kräfte aus einer einzigen Funktion, der

1) Dieses Theorem steht in gewisser Analogie zu einem von M. Hafen angegebenen Integraltheorem [vergleiche dartiber Riemann-Weber, Partielle Differentialgleichungen Bd. I, 5. Aufl., Seiten 201 u. 202] und der Beweis daftir läft sich in ähnlicher Weise, wie dies dort von H. Weber fur das Hafensche Theorem geschehen ist, erbringen.

Um aus

$$
\left.\mathrm{F}(\eta)=\int_{\eta}^{\infty} \frac{f(\xi)}{\sqrt{\xi^{2}-\eta^{2}}} \mathrm{~d} \xi \ldots . . . . . . .1\right)
$$

die Funktion $f$ explizit durch die Funktion $F$ ausdrücken $z u$ können, multiplizieren wir beide Seiten der Gleichung mit $\frac{\eta \mathrm{d} \eta}{\sqrt{\eta^{2}-\mathrm{r}^{2}}}$ und integrieren uber $\eta$ in den Grenzen $\mathrm{r} \div \infty$. Es folgt so:

$$
\int_{\mathbf{r}}^{\infty} \mathrm{F}(\eta) \frac{\eta \mathrm{d} \eta}{\sqrt{\eta^{2}-\mathrm{r}^{2}}}=\int_{\mathbf{r}}^{\infty} \frac{\eta \mathrm{d} \eta}{\sqrt{\eta^{2}-\mathrm{r}^{2}}} \int_{\eta}^{\infty} \frac{\mathrm{f}(\xi)}{\sqrt{\xi^{2}-\eta^{2}}} \mathrm{~d} \xi
$$

Nach der folgenden Regel von M. Hafen [Mathematische Annalen Bd. I9ro, S. 517]:

$$
\int_{\mathbf{r}}^{\mathbf{x}} \mathrm{d} \eta \int_{\eta}^{\mathbf{x}} \Phi(\xi, \eta) \mathrm{d} \xi=\int_{\mathbf{r}}^{\mathbf{x}} \mathrm{d} \xi \int_{\mathbf{r}}^{\xi} \Phi(\xi, \eta) \mathrm{d} \eta
$$

vertauschen wir die Integrationsfolge und erhalten für das Doppelintegral:

$$
\int_{\mathbf{r}}^{\infty} \mathrm{f}(\xi) \mathrm{d} \xi \int_{\mathrm{r}}^{\xi} \frac{\eta \mathrm{d} \eta}{\sqrt{\eta^{2}-\mathrm{r}^{2}} \sqrt{\xi^{2}-\eta^{2}}},
$$
wobei das innere Integral durch die Substitution $\eta^{2}-\mathrm{r}^{2}=\mu^{2}$ gelöst den Wert $\frac{\pi}{2}$ ergibt Es ist
daher:

$$
\frac{\pi}{2} \int_{\mathbf{r}}^{\infty} \mathrm{f}(\xi) \mathrm{d} \xi=\int_{\mathbf{r}}^{\infty} \mathrm{F}(\eta) \frac{\eta \mathrm{d} \eta}{\sqrt{\eta^{2}-\mathrm{r}^{2}}},
$$

woraus durch die Differentation nach $r$ folgt, wenn $g$ eine Konstante darstellt:

$$
\left.\mathbf{f}(\mathrm{r})=-\frac{2}{\pi} \lim _{\mathbf{g}=\infty}\left(\frac{\mathrm{d}}{\mathrm{d} \mathbf{r}} \int_{\mathrm{r}}^{\mathrm{g}} \frac{\mathrm{F}(\eta)}{\sqrt{\eta^{2}-\mathrm{r}^{2}}} \eta \mathrm{d} \eta\right) \ldots . . . . .2\right)
$$

Nach 1) und 2) ist daher das Theorem IV bewiesen. 
sog. „Potentialfunktion“, als partielle Differentialquotienten nach den Koordinatenrichtungen ableiten lassen. Diese Potentialfunktion (skalare Ortsfunktion) läßt sich bekanntlich nach der Fernwirkungstheorie als das folgende Raumintegral über alle elektrischen Mengen $\varrho \cdot d \tau$ des Raumes $\tau$ darstellen:

$$
\left.\varphi=\int_{\tau} \frac{\varrho \mathrm{d} \tau}{\mathrm{r}} . . . . . . . . . \mathrm{I}\right)
$$

$\varrho=$ el. Raumdichte,

$\mathrm{r}=$ Abstand des Aufpunktes vom Quellpunkte.

Man erhält daraus die Komponenten der el. Feldstärke wie folgt:

$$
\left.\mathfrak{E}_{\mathrm{x}}=-\frac{\partial \varphi}{\partial \mathrm{x}}, \quad \mathfrak{E}_{\mathrm{y}}=-\frac{\partial \varphi}{\partial \mathrm{y}}, \quad \mathfrak{E}_{\mathrm{z}}=-\frac{\partial \varphi}{\partial \mathrm{z}} \ldots \ldots .2\right)
$$

Wenn nur im Endlichen elektrische Dichte vorhanden ist, so verschwindet nach I) $\varphi$ im Unendlichen.

Potentialgleichung: Der reziproke Radius $\frac{\mathrm{r}}{\mathrm{r}}$ erfüllt, solange der Aufpunkt des Potentiales außerhalb von allen elektrischen Mengen liegt, die Differentialgleichung:

und deshalb muß auch

$$
\left.\Delta\left(\frac{I}{r}\right)=\frac{\partial^{2}\left(\frac{I}{r}\right)}{\partial x^{2}}+\frac{\partial^{2}\left(\frac{I}{r}\right)}{\partial y^{2}}+\frac{\partial^{2}\left(\frac{I}{r}\right)}{\partial z^{2}}=0 \ldots . . . . .3\right)
$$

$$
\Delta \varphi=\Delta \int_{\tau} \frac{\varrho \mathrm{d} \tau}{\mathrm{r}}=\int_{\tau} \varrho \mathrm{d} \tau \cdot \Delta\left(\frac{\mathrm{I}}{\mathrm{r}}\right)=0
$$

sein. D. h. es gilt für das Potential $p$ die partielle Differentialgleichung:

$$
\left.\Delta \varphi=\frac{\partial^{2} \varphi}{\partial \mathrm{x}^{2}}+\frac{\partial^{2} \varphi}{\partial \mathrm{y}^{2}}+\frac{\partial^{2} \varphi}{\partial \mathrm{z}^{2}}=0, \ldots . . . . .44\right)
$$

der sog. "Laplacesche Operator", der auch als Ausgangspunkt für die Potentialuntersuchungen der Nahwirkungstheorie dient.

Für die in der Schrift behandelten Probleme werden meistens Zylinderkoordinaten verwendet. Es ist dann die Transformation des La placeschen Operators entsprechend den Substitutionen:

$$
\mathrm{x}=\mathrm{r} \cos \Theta, \quad \mathrm{y}=\mathrm{r} \sin \Theta, \quad \mathrm{z}=\mathrm{z} . . . . . \quad \text {. . . 5) }
$$

nötig. In Gl. 4) bleibt dabei $\frac{\partial^{2} \varphi}{\partial \mathrm{z}^{2}}$ ungeändert.

Da nun:

$$
\frac{\partial \mathrm{r}}{\partial \mathbf{x}}=\frac{\mathrm{x}}{\mathrm{r}}=\cos \Theta, \quad \frac{\partial \mathrm{r}}{\partial \mathrm{y}}=\frac{\mathrm{y}}{\mathrm{r}}=\sin \Theta \ldots . . . . .6 \text { ) }
$$

und also:

$$
\operatorname{tg} \Theta=\frac{y}{x}
$$

ist, folgt durch Differentiation:

$$
\begin{aligned}
& \frac{\frac{\partial \theta}{\partial x}}{\cos ^{2} \Theta}=-\frac{y}{x^{2}}=-\frac{\operatorname{tg} \theta}{r \cos \Theta} \\
& \frac{\frac{\partial \theta}{\partial y}}{\cos ^{2} \Theta}=+\frac{I}{x}=+\frac{I}{r \cos \Theta}
\end{aligned}
$$


und somit

$$
\left.\begin{array}{l}
\frac{\partial \Theta}{\partial \mathrm{x}}=-\frac{\sin \Theta}{\mathrm{r}} \\
\frac{\partial \theta}{\partial \mathrm{y}}=+\frac{\cos \Theta}{\mathrm{r}}
\end{array}\right\}
$$

Für die Transformation ist nun zu bilden:

$$
\left.\begin{array}{l}
\frac{\partial \varphi}{\partial \mathrm{x}}=\frac{\partial \varphi}{\partial \mathrm{r}} \cdot \frac{\partial \mathrm{r}}{\partial \mathrm{x}}+\frac{\partial \varphi}{\partial \Theta} \cdot \frac{\partial \Theta}{\partial \mathrm{x}} \\
\frac{\partial \varphi}{\partial \mathrm{y}}=\frac{\partial \varphi}{\partial \mathrm{r}} \cdot \frac{\partial \mathrm{r}}{\partial \mathrm{y}}+\frac{\partial \varphi}{\partial \Theta} \cdot \frac{\partial \Theta}{\partial \mathrm{y}}
\end{array}\right\}
$$

und:

$$
\left.\begin{array}{l}
\frac{\partial^{2} \varphi}{\partial \mathrm{x}^{2}}=\frac{\partial\left(\frac{\partial p}{\partial \mathrm{x}}\right)}{\partial \mathrm{r}} \cdot \frac{\partial \mathrm{r}}{\partial \mathrm{x}}+\frac{\partial\left(\frac{\partial \varphi}{\partial \mathrm{x}}\right)}{\partial \Theta} \cdot \frac{\partial \Theta}{\partial \mathrm{x}} \\
\frac{\partial^{2} \varphi}{\partial \mathrm{y}^{2}}=\frac{\partial\left(\frac{\partial \varphi}{\partial \mathrm{y}}\right)}{\partial \mathrm{r}} \cdot \frac{\partial \mathrm{r}}{\partial \mathrm{y}}+\frac{\partial\left(\frac{\partial \varphi}{\partial \mathrm{y}}\right)}{\partial \Theta} \cdot \frac{\partial \Theta}{\partial \mathrm{y}}
\end{array}\right\}
$$

Nach Ausführung der Differentiationen in 9) mit Berücksichtigung von 8), 6) und 7) folgt nach Zusammenfassung der Glieder und Addition von $\frac{\partial^{2} \varphi}{\partial \mathrm{z}^{2}}$ die transformierte Gleichung:

$$
\left.\frac{\Delta \varphi=\frac{\partial^{2} \varphi}{\partial \mathrm{r}^{2}}+\frac{\mathrm{I}}{\mathrm{r}} \cdot \frac{\partial \varphi}{\partial \mathrm{r}}+\frac{\mathrm{I}}{\mathrm{r}^{2}} \cdot \frac{\partial^{2} \varphi}{\partial \theta^{2}}+\frac{\partial^{2} \varphi}{\partial \mathrm{z}^{2}}}{.} \text {. . . . . . . I } 0\right)
$$

Randbedingungen für die Leiter: Nach 2) und 3) gilt für den freien Äther

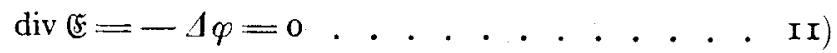
und also für irgendeinen Raumteil $\tau$ desselben nach dem GauBschen Integralsatze:

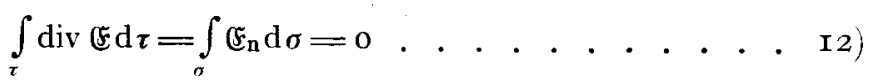

D. h. das über die Berandung $\sigma$ von $\tau$ erstreckte Oberflächenintegral der Normalkomponente der elektrischen Feldstärke ist Null. Betrachtet man nun aber einen Raumteil, in welchem vereinzelte, punktförmige Ladungen vorhanden sind, so läßt sich Gl. I2) noch anwenden, wenn die Massenpunkte nach dem Vorgange der Funktionentheorie durch kleine kugelförmige Begrenzungen vom Integrationsgebiete ausgeschlossen werden. Bezeichnet $\sigma_{\mathrm{A}}$ die äußere Begrenzung des Raumes $\tau$ und $\sigma_{\mathrm{K}}$ die Oberfläche der Kugeln, so muß dann nach I2) sein:

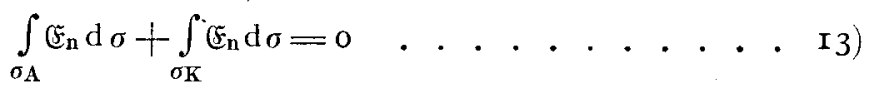

Da nun $\mathfrak{E}_{\mathbf{n}}$ an der Oberfläche irgendeiner Kugel $\boldsymbol{v}$ von der Form ist:

$$
\begin{gathered}
\dot{E}_{\mathrm{n}_{\nu}}=-\frac{\mathrm{e}_{\boldsymbol{v}}}{\mathrm{r}^{2}}+\mathrm{f} \cdot \mathrm{c} . \\
{\left[\mathrm{e}_{\boldsymbol{\nu}}=\right.\text { el. Menge des Punktes], }}
\end{gathered}
$$

wo f.c. eine durch den Punkt kontinuierlich hindurchgehende Funktion bedeutet, während $\left(\frac{\mathrm{e}_{v}}{\mathrm{r}^{2}}\right)$ daselbst wie $\frac{\mathrm{I}}{\mathrm{r}^{2}}$ unendlich wird, läßt sich wegen $\mathrm{d} \sigma_{v}=\mathrm{r}_{\nu}{ }^{2} \mathrm{~d} \omega[\omega=$ Element des Raumwinkels] das Integral über die Kugeln sofort ausführen. Man gewinnt so aus I3) den ,eigentlichen“ GauBschen Satz der Elektrostatik:

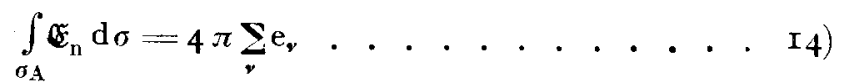


Indem die Summation über die elektrischen Mengen durch die entsprechende Integration ersetzt wird, läßt sich der GauBsche Satz dann auch über kontinuierlich verteilte Massen anwenden. Der Gaußsche Satz leistet uns gute Dienste bei der Aufstellung der für die elektrostatischen Probleme geltenden Randbedingungen an den Leitern.

Erfahrungsgemäß ist die Elektrizität in den Leitern frei beweglich; sie verteilt sich in denselben so, daß im Gleichgewichtszustand die elektrische Kraft überall Null ist. Es ist also dort:

$$
\mathbb{E}=-\operatorname{grad} \phi=0 . . . \quad . \quad . \quad . \quad . \quad . \quad \text { I5) }
$$

$\varphi$ kann also, was in der Tat auch der Fall ist, wenn das Potential nicht identisch verschwinden soll, im Innern der Leiter höchstens konstant sein. Es gilt daher:

I. Randbedingung: Die Leiteroberflächen sind Niveauflächen des Potentiales.

Bedenkt man, daß der Gaußsche Satz I4) für jede beliebige Fläche, die ganz innerhalb eines Leiters verläuft, Gültigkeit hat, so folgt daraus sofort, daß im Leiterinnern keine elektrische Dichte vorhanden sein kann, daß sich die Elektrizität vielmehr vollständig auf den Oberflächen der Leiter ausbreitet.

Die zweite Randbedingung ergibt sich aus dem GauBschen Satz I4), wenn man diesen über eine das Flächenelement $\mathrm{d} \sigma$ der Leiteroberfläche engumschließende Begrenzung anwendet. Nach dem Besprochenen muB die Elektrizität an der Oberfläche der Leiter in dünnen Schichten haften und man kann sie deshalb quantitativ ausdrücken als Produkt eines gewissen Zahlenfaktors, der sog. Flächendichte $\eta$ mit dem zugehörigen Flächenelemente $\mathrm{d} \sigma$. Wird außerdem berücksichtigt, daß die Feldstärke längs des innerhalb des Leiters verlaufenden Teiles der Integrationsfläche Null ist, so folgt aus I4) die Beziehung:

und hieraus die

$$
E_{\mathrm{n}} \mathrm{d} \sigma=4 \pi \eta \mathrm{d} \sigma
$$

II. Randbedingung: In jedem Punkte einer Leiteroberfläche ist:

$$
\left.\Xi_{\mathrm{n}}=-\frac{\partial \varphi}{\partial \mathrm{n}}=4 \pi \eta . . . . . . . . \mathrm{I} 6\right)
$$

\section{Kreislochscheibe.}

Die mathematische Potentialtheorie befaßt sich meistenteils mit Potentialen, die an den Randwertkörpern nicht bloß konstant, sondern gleich willkürlichen Randfunktionen sind. $\mathrm{DaB}$ es in der Tat möglich ist, die Randwerte der Potentiale wenigstens bis auf gewisse additive Konstanten vorzuschreiben, ist der Inhalt der diesbezüglichen Existenzbeweise, die ganz allgemein erbracht worden sind. Die gewöhnlichen Leiterpotentiale sind Spezialfälle dieser allgemeinen Potentiale. Im folgenden gebe ich ein instruktives Anwendungsbeispiel für die sog. „Kreislochscheibe" als Randwertkörper, das uns gestattet, die Lösung in geschlossener Form explizit anzugeben.

Als „Kreislochscheibe“ bezeichne ich eine leitende oder auch nichtleitende Ebene mit kreisrundem Ausschnitte [Radius des Begrenzungskreises = a]. [Vgl. auch Abb. I.] Sie kommt in Kombination mit andern koaxialen Leitern bei manchen praktisch wichtigen Leitersystemen vor. Wir stellen uns nun die Aufgabe auf der Kreislochscheibe für

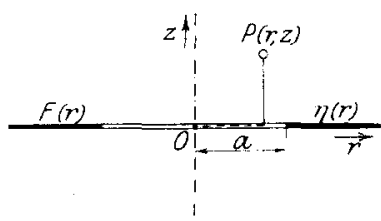

Abb, I. eine willkürlich vorgegebene Funktion $F(r)$ eines axialsymmetrischen Potentiales $\varphi$ die konzentrische Verteiluug $\eta$ der Elektrizität zu finden.

Um diese Aufgabe zu lösen, verwenden wir Zylinderkoordinaten ( $r, z)$ [z-Koordinate senkrecht zur Kreislochscheibe durch deren Mittelpunkt als Anfangspunkt]. Die Niveauflächen des Potentiales müssen der konzentrisch angenommenen Randfunktion 
zufolge Rotationsflächen sein. Als Potentialgleichung dient uns daher die im vorigen Abschnitte hergeleitete Gl. Io), mit der Vereinfachung von $\frac{\partial \varphi}{\partial \Theta}=0$. Es ist dann eine Funktion zu bestimmen, die den folgenden Bedingungen genügt:

I. $\Delta \varphi=\frac{\partial^{2} \varphi}{\partial \mathrm{r}^{2}}+\frac{\mathrm{I}}{\mathrm{r}} \cdot \frac{\partial \varphi}{\partial \mathrm{r}}+\frac{\partial^{2} \varphi}{\partial \mathrm{z}^{2}}=0$

$[\psi$ stetig und o im Unendlichen].

II. $\varphi=\mathrm{F}(\mathrm{r})$ an der Kreislochscheibe [also für: $z=0, \mathrm{r}>\mathrm{a}$ ].

III. Ableitungen von $\varphi$ stetig mit Ausnahme der Normalkomponenten an die Kreislochscheibe.

Da die Kreislochscheibe ganz in der Ebene $z=0$ liegt, ist das Potential $\varphi$ eine ,gerade" Funktion von $z$, und es genügt vollständig, wenn wir $\varphi$ für den Halbraum des positiven $z$ ermitteln. Bedingung III lautet dann dahin, daß:

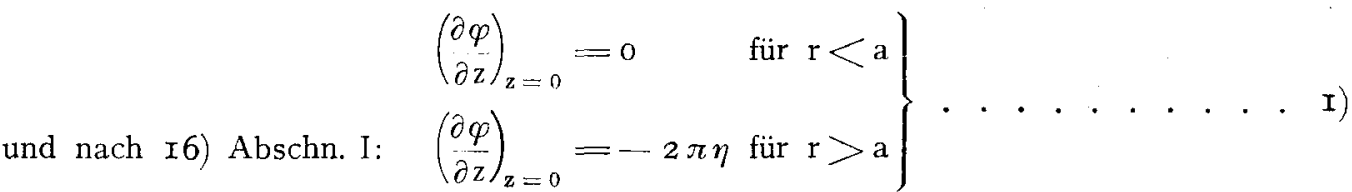
sein muß.

Daß sich Beziehung I6) Abschn. I auch auf diesen allgemeineren Fall, wo der Randwertkörper nicht mehr als Leiter vorausgesetzt ist, übertragen läßt, ergibt sich sofort mit Hilfe des Gaußschen Satzes I4) Abschn. I, der vermöge der gleichen Schlußweise, wie auf S. 5 Gleichung I) bestätigt.

Eine Funktion, die den Bedingungen I und III genügt, ist für positive $z$ :

$$
\left.\varphi=2 \pi \int_{0}^{\infty} \mathrm{e}^{-\alpha \mathrm{z}} \cdot \mathrm{J}(\alpha \mathrm{r}) \mathrm{d} \alpha \int_{\mathrm{a}}^{\infty} \eta(\lambda) \mathrm{J}(c \lambda) \lambda \mathrm{d} \lambda \ldots \ldots . . .2\right)
$$

$\mathrm{DaB}$ die Bedingung III darin enthalten ist, geht aus dem z. B. von P. du BoisReymond bewiesenen Integraltheorem der Besselschen Funktionen nullter Ordnung ${ }^{1}$ ) hervor, das auf diesen Fall spezialisiert lautet:

$$
\left(\frac{\partial \varphi}{\partial z}\right)_{\mathrm{z}=0}=-2 \pi \int_{0}^{\infty} \mathrm{J}(\kappa \mathrm{r}) \alpha \mathrm{d} \alpha \int_{\mathrm{a}}^{\infty} \eta(\lambda) \mathrm{J}(\kappa \lambda) \lambda \mathrm{d} \lambda=\left\{\begin{array}{ll}
0 & \text { für } \mathrm{r}<\mathrm{a} \\
\eta(\mathrm{r}) & \text { für } \mathrm{r}>\mathrm{a}
\end{array} \ldots .3\right)
$$

Der Bedingung II wegen ist nach Gl. 2) zu setzen:

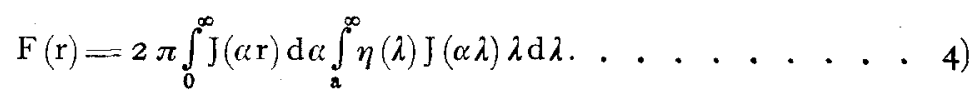

Verwendet man $\mathrm{J}(\alpha \lambda)$ in der Darstellung:

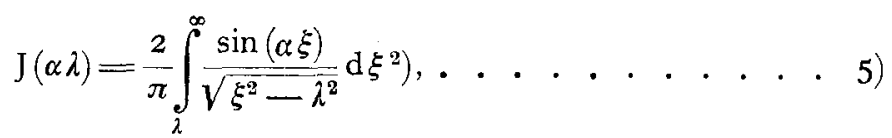

so kann für das innere Integral geschrieben werden:

$$
\int_{\mathbf{a}}^{\infty} \eta(\lambda) \mathrm{J}(\alpha \lambda) \lambda \mathrm{d} \lambda=\frac{2}{\pi} \int_{\mathbf{a}}^{\infty} \eta(\lambda) \lambda \mathrm{d} \lambda \int_{\lambda}^{\infty} \frac{\sin (\alpha \xi)}{\sqrt{\xi^{2}-\lambda^{2}}} \mathrm{~d} \xi .
$$
Formeln.

1) Mathematische Annalen Bd. 4, S. 362. Die Theorie der Fourierschen Integrale und

2) Riemann-Weber, Partielle Differentialgleichungen, Bd. I, S. I8z, Formel 9). 
Rechts kann die Integrationsfolge nach der folgenden Regel vertauscht werden:

$$
\left.\int_{\mathbf{a}}^{\mathbf{x}} \mathrm{d} \lambda \int_{\lambda}^{\mathrm{x}} \Phi(\lambda, \xi) \mathrm{d} \xi=\int_{\mathbf{a}}^{\mathbf{x}} \mathrm{d} \xi \int_{\mathbf{a}}^{\xi} \Phi(\lambda, \xi) \mathrm{d} \lambda^{\mathbf{1}}\right)
$$

Es folgt dann daraus:

$$
\frac{2}{\pi} \int_{a}^{\infty} \sin (\alpha \xi) \mathrm{d} \xi \int_{\mathbf{a}}^{\xi} \frac{\eta(\lambda)}{\sqrt{\xi^{2}-\lambda^{2}}} \lambda \mathrm{d} \lambda
$$

und mit der Bezeichnung von:

$$
\begin{aligned}
& \left.\mathrm{f}(\xi)=4 \int_{a}^{\xi} \frac{\eta(\lambda)}{\sqrt{\xi^{2}-\lambda^{2}}} \lambda \mathrm{d} \lambda \ldots . . . . . . .66\right) \\
& \left.2 \pi \int_{a}^{\infty} \eta(\lambda) \mathrm{J}(\alpha \lambda) \lambda \mathrm{d} \lambda \equiv \int_{a}^{\infty} \mathrm{f}(\xi) \sin (\alpha \xi) \mathrm{d} \xi \ldots . . . .77\right)
\end{aligned}
$$

Wegen 7) gilt nach 4) für $F(r)$ also die Beziehung:

$$
F(r)=\int_{0}^{\infty} J(\alpha r) d \alpha \int_{a}^{\infty} f(\xi) \sin (\alpha \xi) d \xi
$$

und nach Vertauschung der Integrationsfolge:

$$
\left.\mathrm{F}(\mathrm{r})=\int_{\mathbf{a}}^{\infty} \mathrm{f}(\xi) \mathrm{d} \xi \int_{0}^{\infty} \mathrm{J}(\alpha \mathrm{r}) \sin (\alpha \xi) \mathrm{d} \alpha \quad \ldots . . .88\right)
$$

Das innere Integral läßt sich mittelst des Fourierschen Integraltheorems [I, A in der Formeltabelle] leicht auswerten, wenn für $\mathrm{J}(\alpha \mathbf{r})$ das 5$)$ entsprechende Integral eingesetzt wird. Man erhält so:

und damit für 8):

$$
\begin{aligned}
\int_{0}^{\infty} \mathrm{J}(\alpha \mathrm{r}) \sin (\alpha \xi) \mathrm{d} \alpha & =\frac{2}{\pi} \int_{0}^{\infty} \sin (\alpha \xi) \mathrm{d} \alpha \int_{\mathrm{r}}^{\infty} \frac{\sin (\alpha \mu)}{\sqrt{\mu^{2}-\mathrm{r}^{2}}} \mathrm{~d} \mu= \\
& =0 \quad \text { für } \xi<\mathrm{r} \\
& =\frac{\mathrm{I}}{\sqrt{\xi^{2}-\mathrm{r}^{2}}} \text { für } \xi>\mathrm{r}
\end{aligned}
$$

$$
\left.F(r)=\int_{r}^{\infty} \frac{f(\xi)}{\sqrt{\xi^{2}-r^{2}}} d \xi \ldots . . . . . . .99\right)
$$

In dieser Gleichung kann die Funktion $f(\xi)$ mit Hilfe des Theorems IV der Formeltabelle durch die Funktion $F(r)$ leicht ausgedrückt werden. Es muß sein:

$$
\mathrm{f}(\xi)=-\frac{2}{\pi} \cdot \frac{\mathrm{d} \int_{\xi}^{\infty} \frac{\mathrm{F}(\mathrm{r})}{\sqrt{\mathrm{r}^{2}-\xi^{2}}} \mathrm{rdr}}{\mathrm{d} \xi} \ldots . . . . \text { r } \mathrm{r} \text { ) }
$$

Da $f(\xi)$ nun bekannt ist, kann unser elektrostatisches Problem dann als gelöst betrachtet werden, wenn es uns gelingt, in Formel 6) $\eta(2)$ explizit als Funktion von $f(\xi)$ anzugeben. Dazu multiplizieren wir G. 6) auf beiden Seiten mit $\frac{\xi \mathrm{d} \xi}{8 \pi \sqrt{\mathbf{r}^{2}-\xi^{2}}}$ und integrieren über $\xi$ in den Grenzen $a-r$ entsprechend der Gleichung:

$$
\frac{I}{2 \pi} \int_{a}^{r} \frac{f(\xi)}{\sqrt{r^{2}-\xi^{2}}} \xi \mathrm{d} \xi=\frac{2}{\pi} \int_{a}^{r} \frac{\xi \mathrm{d} \xi}{\sqrt{\mathrm{r}^{2}}-\xi^{\xi^{2}}} \int_{a}^{\xi} \frac{\eta(\lambda)}{\sqrt{\xi^{2}-\lambda^{2}}} \lambda \mathrm{d} \lambda .
$$

1) M. Hafen, Mathematische Annalen Bd. I910, S. 517. 
Nach Theorem III der Formeltabelle ist aber das Integral rechts identisch mit:

so daß die Beziehung gilt:

$$
\int_{a}^{r} \eta(r) r d r
$$

$$
\int_{a}^{\mathrm{r}} \eta(\mathrm{r}) \mathrm{rdr}=\frac{\mathrm{I}}{2 \pi} \int_{\mathrm{a}}^{\mathrm{r}} \frac{\mathrm{f}(\xi)}{\sqrt{\mathrm{r}^{2}-\xi^{2}}} \xi \mathrm{d} \xi,
$$

aus welcher durch Differentiation nach $r$ folgt:

$$
\eta(\mathrm{r})=\frac{\mathrm{I}}{2 \pi \mathrm{r}} \cdot \frac{\mathrm{d} \int_{\mathrm{a}}^{\mathrm{r}} \frac{\mathrm{f}(\xi)}{\sqrt{\mathrm{r}^{2}-\xi^{2}}} \xi \mathrm{d} \xi}{\mathrm{dr}} \text {......... II }
$$

Hierdurch ist aber unsere Aufgabe gelöst.

Nach 2) und 7) läßt sich noch das Potential $\varphi$ in Zusammenhang bringen mit der Funktion $f(\xi)$ durch die Gleichung:

$$
\varphi=\int_{0}^{\infty} \mathrm{e}^{-\alpha \mathbf{z}} \cdot \mathrm{J}(\alpha \mathrm{r}) \mathrm{d} \alpha \int_{a}^{\infty} \mathrm{f}(\xi) \sin (\alpha \xi) \mathrm{d} \xi \ldots . . . \text {. . . I2) }
$$

Substituiert man schließlich noch die Funktion $f(\xi)$ aus Gl. Io) in die Gl. II) und I2), so erhält man die gesuchte konzentrische Verteilung $\eta(r)$ der Elektrizität auf der Kreislochscheibe und das Potential $\varphi$ aus der willkürlichen Randfunktion F(r) der letzteren $\mathrm{zu}$ :

$$
\begin{aligned}
& \left.\eta(\mathrm{r})=-\frac{\mathrm{I}}{\pi^{2}} \cdot \frac{\mathrm{I}}{\mathrm{r}} \cdot \frac{\mathrm{d}}{\mathrm{d} \mathrm{r}} \int_{\mathrm{a}}^{\mathrm{r}} \frac{\xi \mathrm{d} \xi}{\sqrt{\mathrm{r}^{2}-\xi^{2}}} \cdot \frac{\int_{\xi}^{\infty} \frac{\mathrm{F}(\varrho)}{\sqrt{\varrho^{2}-\xi^{2}}} \varrho \mathrm{d} \varrho}{\mathrm{d} \xi} \ldots . . . \mathrm{I} 3\right) \\
& \varphi=-\frac{2}{\pi} \int_{0}^{\infty} \mathrm{e}^{-\alpha \mathbf{z}} \cdot \mathrm{J}(\alpha \mathbf{r}) \mathrm{d} \alpha \int_{\mathbf{a}}^{\infty} \sin (\alpha \xi) \mathrm{d} \xi \frac{\mathrm{d} \int_{\xi}^{\infty} \frac{\mathrm{F}(\varrho)}{\sqrt{\varrho^{2}-\xi^{2}}} \varrho \mathrm{d} \varrho}{\mathrm{d} \xi} \ldots \ldots . \text { I4) }
\end{aligned}
$$

Die erhaltenen Resultate sind analog den Lösungen von M. Hafen für die Kreisscheibe $^{1}$ ). Es lassen sich daraus die Ausdrücke der Dichte und des Potentiales in einfacheren Fällen durch Integration vollständig bestimmen. Ein dafür typisches Beispiel behandelt der folgende Abschnitt.

\section{Influenzwirkung eines elektrischen Poles auf die Kreislochscheibe.}

Übernimmt die Kreislochscheibe die Rolle einer leitenden Fläche, so muß sie überall das Potential Null aufweisen. Wenn wir nämlich im Unendlichen keine freie Elektrizität annehmen, so verschwindet nach GI. I), Abschn. I das Potential $\varphi$ dort sicher und also auch an jedem Leiter, der bis dorthin reicht. So z. B. an unserer Kreislochscheibe, die wir als unbegrenzt vorausgesetzt haben. Die Kreislochscheibe braucht deswegen nicht frei von elektrischen Ladungen zu sein, sie wird vielmehr, falls noch geladene Konduktoren oder Massenpole in ihre Nähe gebracht werden, solche Belegungen induzieren, daB Gleichgewicht entsteht; d. h. es wird sich an ihr eine solche

1) Mathematische Annalen Bd. 19เo, S. 522, Formel 8), und 523, Formel (3). 
Massenverteilung einstellen, daß das Potential an allen andern Leitern konstant und insbesondere an der Kreislochscheibe Null wird.

Wir wollen nun in diesem Abschnitt den Fall genauer untersuchen, wo sich im Mittelpunkte der Kreislochscheibe ein elektrischer Pol von der Masse $m$ befindet. Es soll die Potentialfunktion und Dichteverteilung an der Scheibe ermittelt werden. Die Aufgabe läßt sich auf folgende Weise lösen. Man bestimmt zum Potentiale

$$
\left.\varphi_{\mathrm{P}}=\frac{\mathrm{m}}{\sqrt{\mathrm{r}^{2}+\mathrm{z}^{2}}} \cdot \ldots . . . . . . . . \quad \mathrm{r}\right)
$$

des Massenpoles ein zusätzliches Kreislochscheibenpotential mit der Verteilung der induzierten Ladungen und dem entgegengesetzten Randwert des Potentiales $\varphi_{\mathbf{P}}$ an der Scheibe. Da das Potential des Massenpoles sich durch die induzierten Ladungen an der Scheibe in keiner Weise beeinflussen läßt, findet die gestellte Aufgabe also ihre Lösung durch die Resultate des vorhergehenden Abschnittes, wenn man dort

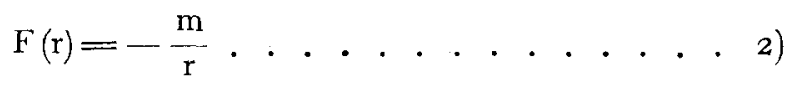

setzt.

Für die Funktion $f(\xi)$, Formel ro), Abschn. II gilt mit Rücksicht auf die Konvergenz des darin vorkommenden Integrales folgender Grenzwert [vergleiche dazu auch Fußnote auf Seite 2]:

$$
\mathrm{f}(\xi)=-\frac{2}{\pi} \lim _{\mathrm{g}=\infty}\left(\frac{\mathrm{d}}{\mathrm{d} \xi} \int_{\xi}^{\mathrm{g}} \frac{\mathrm{F}(\mathrm{r})}{\sqrt{\mathrm{r}^{2}-\xi^{2}}} \mathrm{rdr}\right)
$$

und also für unser Anwendungsbeispiel nach 2):

$$
\lim _{\mathrm{g}=\infty}\left(\frac{\mathrm{d}}{\mathrm{d} \xi} \int_{\xi}^{\mathrm{g}} \frac{\mathrm{dr}}{\sqrt{\mathrm{r}^{2}-\xi^{2}}}\right)=\left|\frac{\mathrm{d} \mathfrak{A r} \mathfrak{C} \mathfrak{o f}\left(\frac{\mathrm{g}}{\xi}\right)}{\mathrm{d} \xi}\right|_{\mathrm{g}=\infty}=-\frac{\mathrm{I}}{\xi} .
$$

Es ist somit:

$$
\left.\mathrm{f}(\xi)=-\frac{2}{\pi} \cdot \frac{\mathrm{m}}{\xi} \cdot \stackrel{\mathrm{g}=\infty}{ } \cdot \ldots . . . . . .3\right)
$$

Nach Gl. I2) des vorigen Abschnittes hat man nun für das Potential $\varphi_{\mathbf{S}}$ der Kreislochscheibe den Ausdruck:

$$
\varphi_{\mathrm{S}}=-\frac{2}{\pi} \mathrm{m} \int_{0}^{\infty} \mathrm{e}^{-\alpha z} \cdot \mathrm{J}(\alpha \mathrm{r}) \mathrm{d} \alpha \int_{\mathrm{a}}^{\infty} \frac{\sin (\alpha \xi)}{\xi} \mathrm{d} \xi \ldots . . . .4 \text {. . . 4) }
$$

Das Integral lautet nach Vertauschung der Integrationsfolge:

$$
\int_{a}^{\infty} \frac{\mathrm{d} \xi}{\xi} \int_{0}^{\infty} \mathrm{e}^{-\alpha z} \cdot \sin (\alpha \xi) \mathrm{J}(\alpha \mathrm{r}) \mathrm{d} \alpha
$$

und kann gedeutet werden als Imaginärteil von:

$$
\left.\int_{a}^{\infty} \frac{\mathrm{d} \xi}{\xi} \int_{0}^{\infty} \mathrm{e}^{-\alpha(\mathrm{z}+\mathrm{i} \xi)} \cdot \mathrm{J}(\alpha \mathrm{r}) \mathrm{d} \alpha=\int_{a}^{\infty} \frac{\mathrm{d} \xi}{\xi \sqrt{\mathrm{r}^{2}+(\mathrm{z}+\mathrm{i} \xi)^{2}}}\right) .
$$

1) Über die Ausführung des inneren Integrales vergleiche man: Riemann-Weber, Partielle Differentialgleichungen Bd. I, S. 194. 

gration:

Indem man im Nenner nach Potenzen von $\xi$ ordnet, findet man nach Inte-

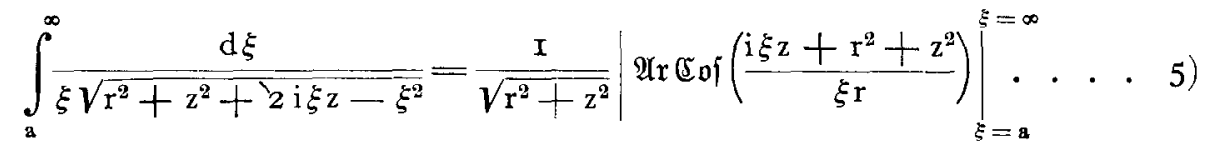

Die obere Grenze liefert:

wovon der Imaginärteil

$$
\frac{I}{\sqrt{r^{2}+z^{2}}} \cdot \mathfrak{A} \mathfrak{C} \mathfrak{E}_{\mathfrak{o j}}\left(\mathrm{i} \frac{z}{\mathrm{r}}\right)
$$

$$
\frac{\pi}{2} \cdot \frac{1}{\sqrt{r^{2}+z^{2}}}
$$

mit den Konstanten $-\frac{2}{\pi} \mathrm{m}$ von 4) multipliziert genau entgegengesetzt gleich dem Potentiale $\varphi_{\mathrm{P}}$ des Poles ist. Es erweist sich also, daß das resultierende Potential:

$$
\varphi=-\varphi_{\mathrm{P}}+\varphi_{\mathrm{S}}
$$

als Imaginärteil der unteren Grenze von 5) als selbständige Funktion durch einen geschlossenen Ausdruck hervorgeht, d. h. das resultierende Potential $\varphi$ ist der Imaginärteil von:

$$
\left.\frac{I}{\sqrt{r^{2}+z^{2}}} \mathfrak{A r} \mathscr{C} \mathfrak{o r}\left(\frac{\mathrm{r}^{2}+z^{2}}{\mathrm{ar}}+\mathrm{i} \frac{z}{r}\right) \mathbf{1}\right)
$$

der nach Multiplikation mit den konstanten Faktoren in 4) das Resultat liefert:

$$
\left.\varphi=\frac{2}{\pi} \cdot \frac{\mathrm{m}}{\sqrt{\mathrm{r}^{2}+\mathrm{z}^{2}}} \cdot \arccos \frac{\sqrt{\mathrm{a}^{2} \mathrm{z}^{2}+\left(\mathrm{r}^{2}+\mathrm{z}^{2}+\mathrm{ar}\right)^{2}}-\sqrt{\mathrm{a}^{2} \mathrm{z}^{2}+\left(\mathrm{r}^{2}+\mathrm{z}^{2}-\mathrm{ar}\right)^{2}}}{2 \mathrm{ar}} \cdot 6\right)
$$

Daß die gefundene Funktion den Bedingungen des gesuchten Potentiales genügt, ist folgendermaßen einzusehen. Erstens ist $\mathrm{zu}$ bemerken, daB sie notwendigerweise die Laplacesche Gleichung $\Delta \varphi=0$ erfüllt, da dies für jede Funktion von der Form

$$
\int_{0}^{\infty} \mathrm{e}^{-\alpha \mathrm{z}} \cdot \mathrm{J}(\alpha \mathrm{r}) \cdot \Phi(\alpha) \mathrm{d} \alpha
$$

zutrifft. Es erübrigt also bloß zu zeigen, daß die Funktion Gl. 6) den Randbedingungen entsprechend ist, die für unser Problem folgendermaßen lauten:

$$
\text { I. } \varphi=\frac{\mathrm{m}}{\sqrt{\mathrm{r}^{2}+\mathrm{z}^{2}}}+\mathrm{f} \text {. c. am Massenpole }
$$

[f. c. bedeutet eine im Punkte $P(r=0, z=0)$ reguläre Funktion].

$$
\begin{aligned}
& \text { II. }(\varphi)_{\mathrm{z}=0} \neq 0 \quad \text { für } \mathrm{r}<\mathrm{a} \\
& \text { III. }\left(\frac{\partial \varphi}{\partial \mathrm{z}}\right)_{\mathrm{z}=0}=0 \quad \text { für } \mathrm{r}<\mathrm{a} \\
& =2 \pi \eta(\mathrm{r}) \quad, \quad \mathrm{r}>\mathrm{a} \text {. }
\end{aligned}
$$

Der Bedingung I zufolge muß, wie es einer in beliebiger Richtung erfolgenden Annäherung an den Massenpol entspricht, $\varphi$ im Punkte $P(r=0, z=0)$ wie der

1) Es ist bekanntlich der Imaginärteil von $\mathfrak{A} \mathfrak{C} \mathfrak{0} j(x+i y)$

$$
\pm \arccos \frac{\sqrt{(1+x)^{2}+y^{2}}-\sqrt{(1-x)^{2}+y^{2}}}{2} .
$$

Siehe z. B. Laska, Formelsammlung Seite II. 
reziproke Abstand $\frac{\mathrm{I}}{\sqrt{\mathrm{r}^{2}+\mathrm{z}^{2}}}$ des Aufpunktes $\infty$ werden. Wir wollen annehmen, der Weg, längs dessen wir uns in einer beliebigen Meridianebene gegen den Punkt $\mathrm{P}$ bewegen, sei gegeben durch die Gleichung der Geraden:

$\mathrm{z}=\mathrm{Q}^{\mathrm{r}}$, . . . . . . . . . . . . . 7)

wobei $\varrho$ den sog. Richtungskoeffizienten bedeutet. Wir substituieren diesen Wert von $z$ in das Resultat 6). Unsere Beweisführung läuft dann dahinaus, zu zeigen, daß beim Grenzübergang zu $\mathrm{r}=0$ der arc cos jener Formel für jeden beliebigen Wert von $\varrho$ stetig gegen $\frac{\pi}{2}$ konvergiert. Die Substitution 7) liefert für den arc cos in 6) nach Kürzung mit $r$ :

$$
\arccos \frac{\sqrt{\mathrm{a}^{2} \varrho^{2}+\left[\mathrm{r}\left(\mathrm{I}+\varrho^{2}\right)+\mathrm{a}\right]^{2}}-\sqrt{\mathrm{a}^{2} \varrho^{2}+\left[\mathrm{r}\left(\mathbf{I}+\varrho^{2}\right)-\mathrm{a}\right]^{2}}}{2 \mathrm{a}},
$$

der stetig gegen $\frac{\pi}{2}$ konvergiert, wenn die Differenz der beiden Wurzeln stetig gegen 0 abnimmt. Dies ist in der Tat für $\lim \mathrm{r}=0$ bei

$$
0 \leq \underline{0}<\infty
$$

der Fall, weil für kleine $r$ stets

$$
\mathbf{r}\left(\mathbf{r}+\varrho^{2}\right) \mid \text { gegen } \mid \mathbf{a}
$$

vernachlässigt werden darf. $\mathrm{DaB}$ auch die obere Grenze $\infty$ ton $\varrho$ keine Ausnahme dafür ergibt, erkennt man sofort, wenn man beim Potentiale $\varphi$ Formel 6) zur Grenze $\mathbf{r}=0$ und nachträglich noch zur Grenze $\mathrm{z}=0$ übergeht. Beim Grenzübergang zu $\mathrm{r}=\mathrm{o}$ tritt im Argument des arc cos von Formel 6) die unbestimmte Form $\frac{0}{0}$ auf, deren Auswertung folgendes liefert:

$$
(\varphi)_{\mathrm{r}=0}=\frac{2}{\pi} \cdot \frac{\mathrm{m}}{\mathrm{z}} \cdot \operatorname{arctg}\left(\frac{\mathrm{a}}{\mathrm{z}}\right),
$$

woraus:

$$
\lim _{\mathbf{z}=0}(\varphi)_{r=0}=\left|\frac{\mathrm{m}}{\mathrm{z}}\right|_{\mathbf{z}=0} .
$$

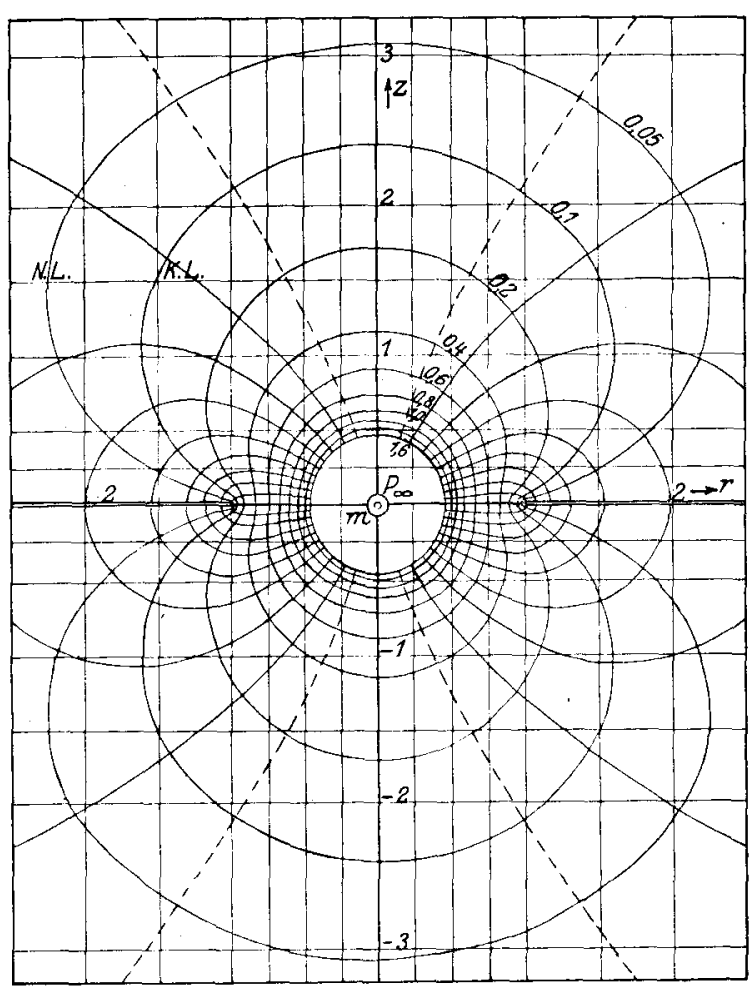

Abb. 2.

$\varphi$ ist also sicher in der Nähe des

Massenpoles, wie es der Bedingung I entspricht, von der Form:

$$
\frac{\mathrm{m}}{\sqrt{\mathrm{r}^{2}}+\mathrm{z}^{2}}+\mathrm{f} \text {. c. }
$$

Um Bedingung II nachzuweisen, machen wir den Grenzübergang von $p$ zu $z=0$. Aus 6) folgt:

$$
(\varphi)_{\mathrm{z}=0}=\frac{2}{\pi} \cdot \frac{\mathrm{m}}{\mathrm{r}} \cdot \arccos \frac{(\mathrm{r}+\mathrm{a}) \pm(\mathrm{r}-\mathrm{a})}{2 \mathrm{a}},
$$

wobei das positive Zeichen für $r<a$ und das negative für $r>a$ zu verwenden ist. 
Also ist:

$$
\begin{aligned}
(\varphi)_{\mathbf{z}=0} & =\frac{2}{\pi} \cdot \frac{\mathrm{m}}{\mathrm{r}} \cdot \operatorname{arc} \cos \left(\frac{\mathrm{r}}{\mathrm{a}}\right) ; \\
& =0 \quad \text { für } \mathrm{r}<\mathrm{a} \\
& \quad ; \text { für } \mathrm{r}>\mathrm{a} .
\end{aligned}
$$

Die Bedingung III kann man leicht durch Differentiation von $\varphi$ nach $z$ und nachherige Limesbildung bestätigen. Es erweist sich dabei tatsächlich, daß $\left(\frac{\partial \varphi}{\partial z}\right)_{z=0} i m$ Intervalle $0<\mathrm{r}<\mathrm{a}$ verschwindet. Zugleich erhält man so den Ausdruck für die elektrische Dichte an der Scheibe, die man in Übereinstimmung mit jener Berechnung aber rascher mit Formel II) des vorhergehenden Abschnittes ermittelt. Danach ist:

wobei nach 3):

$$
\left.\eta(\mathrm{r})=\frac{\mathrm{I}}{2 \pi \mathrm{r}} \mathrm{dr} \int_{\mathrm{a}}^{\mathrm{r}} \mathrm{f}(\xi) \frac{\xi \mathrm{d} \xi}{\sqrt{\mathrm{r}^{2}-\xi^{2}}}, \ldots . . . . .8\right)
$$

$$
f(\xi)=-\frac{2}{\pi} \cdot \frac{m}{\xi}
$$

ist, und es handelt sich also um die Auswertung von:

$$
\frac{d}{d r} \int_{a}^{r} \frac{d \xi}{\sqrt{r^{2}-\xi^{2}}}=\frac{d\left(\arccos \frac{a}{r}\right)}{d r}=\frac{a}{r \sqrt{r^{2}-a^{2}}} .
$$

Multipliziert man diesen Ausdruck mit den Faktoren von 8) und $f(\xi)$, so folgt für die elektrische Dichte $\eta(\mathrm{r})$ an der Kreislochscheibe das einfache Resultat:

$$
\eta(\mathrm{r})=-\frac{\mathrm{m}}{\pi^{2}} \cdot \frac{\mathrm{a}}{\mathrm{r}^{2} \sqrt{\mathrm{r}^{2}-\mathrm{a}^{2}}} \text {. . . . . . . . . . 9) }
$$

Die Gesamtelektrizität der Scheibe findet man durch Integration über die Dichte wie folgt:

$$
\mathfrak{q}=\int_{(S)} \eta \mathrm{d} \sigma=\int_{\mathrm{a}}^{\infty} \int_{0}^{2 \pi} \eta(\mathrm{r}) \mathrm{r} \cdot \mathrm{d} \mathrm{r} \mathrm{d} \gamma
$$

Es ist also nach 8) allgemein:

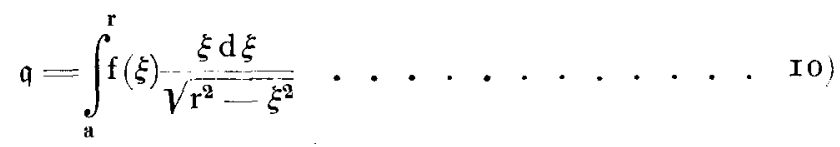

und in unserem speziellen Falle:

d. h.

$$
\mathrm{q}=-\frac{2}{\pi} \mathrm{m}\left|\arccos \left(\frac{\mathrm{a}}{\mathrm{r}}\right)\right|_{\mathrm{a}}^{\infty},
$$

$$
q=\cdots m \text {. }
$$

Es zeigt sich also, daß, wie zu erwarten war, die an der Kreislochscheibe durch den Pol aufgesogene Elektrizitätsmenge entgegengesetzt gleich der Menge des Poles ist.

Der in Formel 6) gefundenen Potentialfunktion entspricht ein Kraftlinienbild, wie es Abb. 2 für einen Meridianschnitt veranschaulicht. Über die Konstruktion des Bildes und für zugehörige Schnitte durchs Potentialfeld vergleiche man im Anhange S. 46 und 47 . 


\section{Vervollständigte Theorie der linearen Leiter für das Vakuum.}

Die Aufstellung der Gesetze für das elektromagnetische Feld von linearen Leitern im Vakuum verdanken wir verschiedenen Autoren. So rührt das Elementargesetz für den magnetischen Bestandteil desselben von Biot und Savart her, und Franz Neumann hat uns durch Einführung des sog. ,elektrodynamischen Potentiales“ die Möglichkeit geschaffen, die längs eines beliebigen Integrationsweges durch lineare Ströme ,induzierte Spannung“ in einfacher Weise auszudrücken. Außerdem sind ron Ampère und manchen andern Autoren noch eine ganze Reihe solcher Elementargesetze angegeben worden, die in ihrer Anwendung auf geschlossene Ströme im Einklange mit den obengenannten Gesetzen stehen. Den üblichen Ableitungen und Darstellungen der Gesetze mangelt aber ihrer stückweisen Entstehung wegen die Einheitlichkeit. Außerdem ist zu bemerken, daß sich in der Literatur nirgends eine vollständige Darstellung der.Bestandteile findet, die den gesamten elektromagnetischen Zustand ausmachen. So fehlt z. B. überall die genaue Formulierung für diejenigen Feldteile, welche durch den ,Ohmschen Spannungsabfall" und durch die mitgeführte Elektrizitätsmenge, hervorgerufen werden. Die Gleichungen der genannten Autoren sind von mir nach dieser Richtung hin vervollständigt worden. Außerdem habe ich danach getrachtet, die Theorie der linearen Leiter unter etwas allgemeineren Voraussetzungen, als dies bis jetzt geschehen ist, zu behandeln. Hauptsächlich ist zu beachten, daß sämtliche Gesetze aus denjenigen von Leitern mit endlichem Querschnitt durch entsprechende Grenzübergänge gewonnen worden sind. Es bietet daher keine Schwierigkeiten, die für die ideellen linearen Leiter geltenden Gesetze in der richtigen Weise als Näherungsgesetze auf Leiter mit endlichen und nur hinlänglich kleinen Querschnitten zu übertragen. Auch dem Umstande, daB die Leitungsdrähte heute vielfach aus Eisen hergestellt werden, ist durch Einführung einer fiur die Leiter vom umgebenden Medium verschiedenen Permeabilität Rechnung getragen worden. Es hat sich dabei gezeigt, daß die induzierte elektrische Feldstärke unter den vorliegenden Verhältnissen ein nicht zu vernachlässigendes Korrektionsglied erhält, während der magnetische Zustand des Feldes außerhalb der Leiter ungeändert bleibt.

Meine Untersuchung hat zudem ergeben, daß man bei der Formulierung der Elementargesetze des magnetischen und elektrischen Feldes mit Vorteil von der Einführung des sog. ,Vektorpotentiales" absieht. Dem Vektorpotentiale geht ja ohnehin die Eigenschaft, welche die statischen Potentiale so wertvoll macht, daß die Komponenten der Kraft daraus als partielle Differentialquotienten abgeleitet werden können, vollständig ab. Die Bezeichnung - Potential ... rührt hier lediglich davon her; daß die Komponenten des Vektorpotentiales formell derselben Differentialgleichung, der sog. Poissonschen genügen, wie die statischen Potentiale.

Bei unseren Betrachtungen gehen wir aus von den Maxwellschen Feld-und zugehörigen Ergänzungsgleichungen. Bei der Integration machen wir die Annahme, daß der Bestandteil der magnetischen Feldstärke, welcher durch die zeitliche Änderung des elektrischen Feldes hervorgerufen wird, vernachlässigbar sei. Dies ist bekanntlich für alle Vorgänge auf dem Gebiete der Elektrotechnik mit fast absoluter Genauigkeit zulässig. Von hervortretendem Einfluß wird das erwähnte zusätzliche Glied erst bei Hochfrequenz, also z. B. für die Erscheinungen an den Antennen bei der drahtlosen Telegraphie. Zudem mache ich die Voraussetzung, die beim Grenzübergang vom endlichdimensionierten zum linearen Leiter mit den wirklichen Verhältnissen vereinbar ist, daß $\mu$ im Leiterinnern nur eine Funktion der Zeit und nicht des Ortes ist.

Entsprechend unserer ersten Annahme lauten die Maxwellschen Feld- und Ergänzungsgleichungen: 


$$
\begin{array}{ll|ll}
\text { I. } & \operatorname{rot} \mathfrak{S}=4 \pi \mathrm{j} & \operatorname{div} \mathfrak{D}=4 \pi \varrho & \mathrm{I}^{\prime} . \\
\text { II. } & \operatorname{rot} \mathfrak{E}=-\frac{\partial \mathfrak{B}}{\partial \mathrm{t}} & \operatorname{div} \mathfrak{B}=0 & \text { II'. }^{\prime} .
\end{array}
$$

$$
\left.\begin{array}{l}
\mathfrak{S}=\text { magnetischer } \\
\mathfrak{E}=\text { elektrischer }
\end{array}\right\} \text { Feldvektor, } \quad \begin{aligned}
& \mathfrak{B}=\text { magn. Induktion, } \quad \mathrm{j}=\text { Stromdichte }, \\
& \mathfrak{D}=\text { elektr. Verschiebung, } \quad \varrho=\text { elektr. Raumdichte, } \\
& \mathrm{t}=\text { Zeit. }
\end{aligned}
$$

Außerdem sollen die Materialgleichungen gelten:

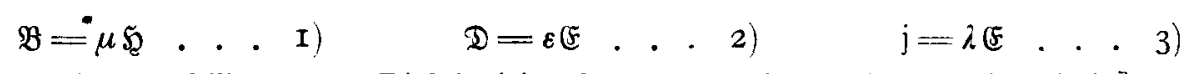

[ $\mu=$ magn. Permeabilität; $\varepsilon=$ Dielektrizitätskonstante; $j=$ elektr. Leitfähigkeit.]

Um Gleichung I allgemein zu integrieren, bilden wir:

$$
\left.\operatorname{rot} \operatorname{rot} \mathfrak{H}=\operatorname{grad} \operatorname{div} \mathfrak{Q}-\nabla^{2} \mathfrak{H}=4 \pi \operatorname{rot} j . . . . . .44\right)
$$

In dem die Leiter umgebenden Vakuum ist $\mu=\mathrm{I}$ und im Leiter bezeichnen wir die Permeabilität als konstante Ortsfunktion mit $\mu$. Dann ist div $\mathfrak{G}$ im Leiteraußen- und -innenraum sicher gleich Null, und Gl. 4) nimmt die Form an:

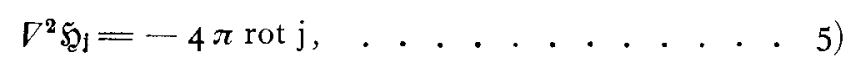

welche für die einzelnen Komponenten von $\mathfrak{h}$ formell übereinstimmt mit der Poissonschen Gleichung der Elektrostatik. Sie hat demnach für $\mathfrak{B}$ das Integral:

$$
\mathfrak{E}_{\mathrm{j}}=\int_{\boldsymbol{\tau}} \frac{\operatorname{rot} \mathrm{j}}{\mathrm{r}} \mathrm{d} \tau \text {. . . . . . . . . . . . . . . . . . 6) }
$$

$[\mathrm{r}=$ Abstand des Quellpunktes $\mathrm{Q}(\mathrm{x}, \mathrm{y}, \mathrm{z})$ im stromdurchflossenen Raumelement $\mathrm{d} \tau$ vom Aufpunkte $\mathrm{P}(\mathrm{a}, \mathrm{b}, \mathrm{c})$ für $\mathfrak{S}]$.

Aus der Analogie mit den für die Statik geltenden Verhältnissen schließen wir, daß $\mathfrak{S}_{\mathbf{x}}, \mathfrak{H}_{\mathbf{y}}, \mathfrak{S}_{z}$ vollständig stetig sind und selbst in den stromdurchflossenen Leiterelementen keine Sprünge erleiden.

Gl. 6) kann'noch durch partielle Integration auf andere Form gebracht werden. Es ist:

und:

$$
\operatorname{rot}_{x} j=\frac{\partial j_{z}}{\partial y}-\frac{\partial j_{y}}{\partial z}
$$

Da aber:

$$
\frac{\mathrm{r}}{\mathrm{r}} \cdot \frac{\partial \mathrm{j}_{\mathrm{z}}}{\partial \mathrm{y}}=\frac{\partial}{\partial \mathrm{y}}\left(\frac{\mathrm{j}_{\mathrm{z}}}{\mathrm{r}}\right)+\frac{\mathrm{j}_{\mathrm{z}}}{\mathrm{r}^{2}} \cdot \frac{\partial \mathrm{r}}{\mathrm{dy}}
$$

$$
\frac{\partial \mathrm{r}}{\partial \mathrm{y}}=\frac{\mathrm{y}-\mathrm{b}}{\mathrm{r}}=-\mathrm{r}_{1 \mathrm{y}}
$$

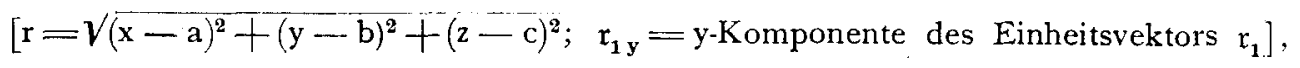

ist, gilt:

$$
\left.\frac{\operatorname{rot}_{x} j}{r}=\frac{\partial}{\partial y}\left(\frac{j_{z}}{r}\right)-\frac{\partial}{\partial z}\left(\frac{j_{y}}{r}\right)+\frac{j_{y} \cdot r_{1 z}}{r^{2}}-\frac{j_{z} \cdot r_{1} y}{r^{2}} \ldots . . .7\right)
$$

Das Raumintegral über die beiden ersten Terme rechts kann umgeformt werden in ein Flächenintegral über die Begrenzung dieses Raumes entsprechend ${ }^{\mathbf{1}}$ ):

$$
\begin{gathered}
\int_{\boldsymbol{t}}\left\{\frac{\partial}{\partial \mathrm{y}}\left(\frac{\mathrm{j}_{\mathrm{z}}}{\mathrm{r}}\right)-\frac{\partial}{\partial z}\left(\frac{\mathrm{j}_{\mathrm{y}}}{\mathrm{r}}\right)\right\} \mathrm{d} \boldsymbol{\tau}=\int_{\sigma}\left\{\frac{\mathrm{j}_{\mathbf{z}}}{\mathrm{r}} \cos (\mathrm{ny})-\frac{\left.\mathrm{j}_{\mathrm{y}_{-}} \cos (\mathrm{nz})\right\} \mathrm{r} \sigma}{\mathrm{r}}\right. \\
{[\mathrm{n}=\text { Normale im Flächenelement } \mathrm{d} \sigma] .}
\end{gathered}
$$

1) Vgl. Drude, Physik des Äthers, 2. Aufl., Verlag von Ferdinand Enke, Stuttgart, S. 32, Formel 16). 
Unter Einführung des Einheitsvektors $n_{1}$ in der Richtung der Normalen $n$ und Berücksichtigung, daß die Leiteroberfläche $\sigma$ durch Stromlinien begrenzt ist, folgt dafür:

$$
\int_{\sigma} \frac{\left[\mathrm{n}_{1}, \mathrm{j}\right]}{\mathrm{r}} \mathrm{d} \sigma \quad \mathrm{o} .
$$

$\mathrm{DaB}$ das Integral tatsächlich verschwindet, erkennt man sofort, wenn man sich den Übergang des Leiters in den nichtleitenden Teil statt diskontinuierlich, rasch aber stetig denkt. Von 7) bleibt also für das Raumintegral bloß die Komponente:

wonach:

$$
\begin{aligned}
& \mathfrak{H}_{j, x}=\int \frac{j_{y} \cdot x_{1 z}-j_{z} \cdot r_{1 y}}{r^{2}} d \tau,
\end{aligned}
$$

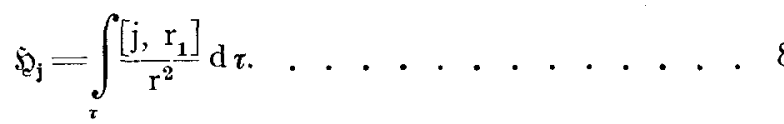

Gehen wir nun zu einem sehr dünnen Leiter über, wo:

$$
\mathrm{jd} \boldsymbol{\tau}=(\mathrm{jdf}) \mathrm{ds}=\mathrm{ids} \quad \text { ist, }
$$

[df $=$ Querschnitt eines Leiterelementes von der Länge $\mathrm{ds}, \mathrm{i}=$ Strom durch $\mathrm{df}$ ] so liefert in 8) die Auswertung des Vektorproduktes für den Integranden:

$$
\begin{gathered}
\left.\mathrm{d} \mathfrak{S}:=\frac{\mathrm{i} \sin \gamma \mid \mathrm{ds}}{\mathrm{r}^{2}}, \ldots \ldots \ldots \ldots\right) \\
{[\gamma=\Varangle(\mathrm{i}, \mathrm{r})]}
\end{gathered}
$$

also nichts anderes als das Biot-Savartsche Elementargesetz.

Wir wollen nun $\mathfrak{g}$ als Funktion des Abstandes $\varrho$ von irgendeinem Punkte $\mathrm{L}$ eines stetig gekrümmten, stromdurchflossenen linearen Leiters bestimmen [vergleiche Abb. 3]. Wir denken uns für $\mathfrak{H}$ zwei Beiträge, wovon der eine von dem $\mathfrak{G}$ benachbarten Leiterelemente $(-\mathrm{h}$, + h) und der andere von den übrigen Elementen der Strombahn herrühren möge. Auf Grund des Gesetzes 9) folgt für den ersten Bei$\operatorname{trag} \overline{\mathfrak{g}}$ von $\mathfrak{\mathfrak { g }}$ :

$$
|\overrightarrow{\mathfrak{G}}|=i \int_{-\mathrm{h}}^{+\mathrm{h}} \frac{\sin \gamma \mathrm{d} \mathrm{s}}{\mathrm{r}^{2}}=\mathrm{i} \varrho \int_{-\mathrm{h}}^{+\mathrm{h}} \frac{\mathrm{d} \mathrm{s}}{\mathrm{r}^{3}}=\frac{2 \mathrm{i}}{\varrho} \cdot \frac{\mathrm{h}}{\mathrm{r}_{\mathrm{p}}},
$$

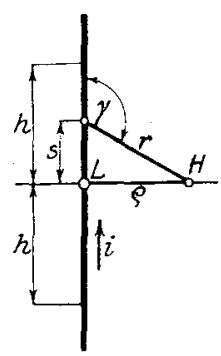

Abb. 3 .

woraus im Grenzwert:

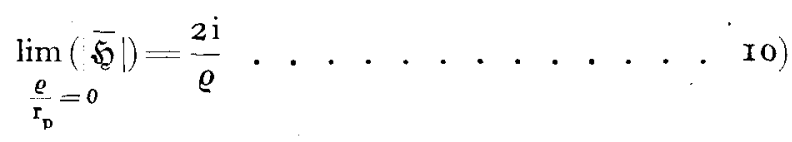

Der Integralbeitrag der übrigen Leiterelemente ist eine für den Punkt $\mathrm{H}$ endlich bleibende Funktion (functio continua). Also gilt für die magnetische Feldstärke in der Nähe des Punktes L:

$$
\left.|\mathfrak{g}|=\frac{2 \mathrm{i}}{\varrho}+\mathrm{f} . \mathrm{c} \ldots . . . . . . \mathrm{r} \mathbf{x}\right)
$$

und nach 8) steht der Vektor $\mathfrak{g}$ zum Leiterelement $(-\mathrm{h},+\mathrm{h})$ windschief normal im Sinne einer Rechtsschraube.

Aus II) schließen wir, daß die magnetischen Kraftlinien Drehkreise um die Leiterachse sind, und zwar sowohl innerhalb als auch in nächster Nähe außerhalb des Leiters. Da $\operatorname{div} \mathfrak{\mathfrak { G }}$ für ein solches Feld ganz unabhängig von der Konzentration der Kraftlinien Null ist, folgt daraus entschieden, daß dies für die Leiteroberfläche, wofür wir bis jetzt noch nichts ausgesagt hatten, in diesem Sonderfalle auch zutrifft. Flächenbelegungen, die ein Magnetfeld im Sinne der Statik erzeugen wïrden, können nicht auftreten. 
Nach Ir) besteht Proportionalität der Feldstärke $\mathfrak{H}$ zum Strom i. Da aber nach I wegen:

$$
\begin{aligned}
\operatorname{div} \operatorname{rot} \mathfrak{g} & =0 \\
\operatorname{div} \mathrm{j} & =0
\end{aligned}
$$

ist, haben wir längs des ganzen Leiters denselben Strom und nach II) die gleichen Verhältnisse für $\mathfrak{\mathfrak { b }}$. Daraus folgt, daß für ein homogenes Leitermaterial [ $\mu$ kann eine Funktion von $\mathfrak{\mathfrak { B }}$ sein] die Permeabilität keine Funktion der Längskoordinate des Leiters sein kann. $\mu$ könnte höchstens noch in radialer Richtung eine Ortsfunktion sein. Wie leicht ersichtlich ist, läßt sich aber im Grenzfalle des linearen Leiters auch unter dieser Voraussetzung ein in radialer Richtung konstantes $\mu$ stets so definieren, daß der Induktionsfluß die richtige Größe hat. $\mu$ ist dann, wie wir früher angenommen haben, im Leiter. keine Orts-, sondern nur noch Zeitfunktion.

Die Bestandteile des elektrischen Feldes finden wir durch Integration der Maxwellschen Feldgleichung II. Wir bilden:

$$
\left.\operatorname{rot} \operatorname{rot} E=\operatorname{grad} \operatorname{div} E-\nabla^{2} \mathfrak{E}=-\frac{\partial}{\partial \mathrm{t}} \operatorname{rot} \mathfrak{B} \ldots \ldots \text {. . . r } 3\right)
$$

Nun ist nach $I^{\prime}$ und 2):

$$
\operatorname{div} \mathfrak{D}=\operatorname{div} \varepsilon \mathscr{E}=4 \pi \underline{ }
$$

Im Vakuum ist $\varepsilon=\mathrm{I}$ und $\varrho=0$; also verschwindet $\operatorname{div} \&$ im Leiteraußenraum sicher. Im Leiterinnern ist $\varepsilon$ konstant und stellt eine sehr große Zahl dar, die praktisch als $\infty$ angenommen werden darf; zudem ist dort $\varrho$ sehr klein, so daß mit großer Genauigkeit: $\operatorname{div} \varepsilon=\frac{4 \pi \varrho}{\dot{\varepsilon}} \simeq 0$ gesetzt werden kann.

Es gilt also für den Leiteraußen- und -innenraum $\operatorname{div} E=0$. Nur an der Leiteroberfläche trifft dies, wie wir zeigen werden, nicht mehr zu; die Feldstärke \& erleidet dort eine Unstetigkeit. Wir machen nun die folgende, für die einzelnen Komponenten algebraisch zu verstehende Zerlegung:

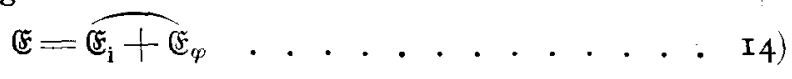

und verstehen unter $\mathscr{E}_{i}$ denjenigen Anteil von $\mathfrak{E}$, der direkt durch die zeitliche Variation des Induktionsflusses entsteht, während $\Xi_{q}$ bloß durch Massenbelegungen an der Leiteroberfläche hervorgerufen sein soll. Es gelten dann nach r3) die Differentialgleichungen:

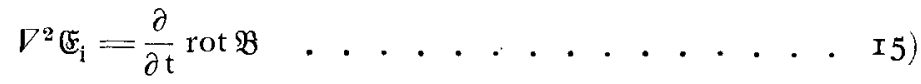

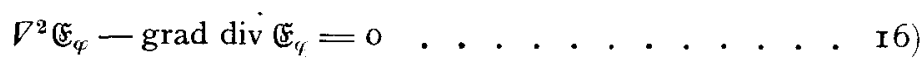

$$
\begin{aligned}
& \text { Nun ist aber: } \quad \operatorname{rot} \mathfrak{B}=\mu \operatorname{rot} \mathfrak{g}-[\mathfrak{S}, \operatorname{grad} \mu]
\end{aligned}
$$$$
\text { und nach I: } \quad \operatorname{rot} \mathfrak{Y}=4 \pi \mathrm{j} \text {, }
$$

so daß I5) geschrieben werden kann:

$$
\nabla^{2} \mathbb{E}_{\mathrm{i}}=\frac{\partial}{\partial \mathrm{t}}\{4 \pi \mathrm{j} \mu-[\mathfrak{g}, \operatorname{grad} \mu]\} .
$$

Das allgemeine Integral dieser Gleichung lautet:

$$
\mathfrak{E}_{\mathrm{i}}=-\frac{\partial}{\partial \mathrm{t}} \int_{\tau} \frac{\mathrm{j} \mu}{\mathrm{r}} \mathrm{d} \tau+\frac{\mathrm{I}}{4 \pi} \frac{\partial}{\partial \mathrm{t}} \int_{\tau} \frac{[\mathscr{\mathfrak { L }}, \operatorname{grad} \mu]}{\mathrm{r}} \mathrm{d} \tau \ldots . . . \text {. I7) }
$$

[ $\mathbf{r}=$ Abstand des Aufpunktes vom Quellpunkte].

Das Integral I7) gilt unter sehr allgemeinen Voraussetzungen; auch dann noch, wenn Körper verschiedenartiger Permeabilität im Raume vorhanden sind. $\mu$ kann 
selbst eine willkürliche Funktion des Ortes und der Zeit sein. Doch müßte in diesem Falle die Verteilung von $j$ und $\mathfrak{S}$ für jeden Zeitmoment im ganzen Raume bekannt sein, was nur im Zusammenhange mit der ersten Maxwellschen Gleichung zu erreichen wäre.

Wir wollen uns hier mit dem Falle weiter befassen, der für lineare Leiter von beliebiger Permeabilität ein Interesse hat; nämlich wo $\mu$ in einzelnen Körpern nur eine Funktion der Zeit und nicht des Ortes ist. Unter dieser Voraussetzung gehen aus dem zweiten Integrale in I7) Flächenintegrale über die Begrenzungen der einzelnen magnetisierbaren Medien hervor. Im Innern dieser Medien verschwindet nämlich grad $\mu$, während dafür an den Oberflächen sprunghafte Änderungen auftreten.

Bedeutet $\mathrm{d} \sigma$ ein Flächenelement und $n_{1}$ den Einheitsvektor in Richtung der Normalen $n$ desselben irgendwo an der Begrenzungsfläche eines solchen magnetisierbaren Körpers, so lautet das dem zweiten Raumintegral in I 7 ) entsprechende Integral über diese Fläche [Vektor $\Im]$ :

$$
\mathfrak{J}=\frac{\partial}{\partial \mathrm{t}}\left\{\frac{\mu-\mathrm{I}}{4 \pi} \int_{\sigma} \frac{\left[\mathfrak{S}, \mathrm{n}_{1}\right]}{\mathrm{r}} \mathrm{d} \sigma\right\}
$$

$[\mu$ - I gleich Sprung der Permeabilität $\mu$ des Körpers in die Permeabilität I des

Wir bezeichnen mit umgebenden Vakuums].

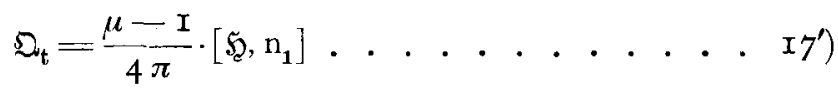

den zufolge der Unstetigkeit der Permeabilität tangential in der Trennfläche $\sigma$ auftretenden Vektor, dem die Bedeutung eines „Oberflächenstromes“ zukommt. Damit ist:

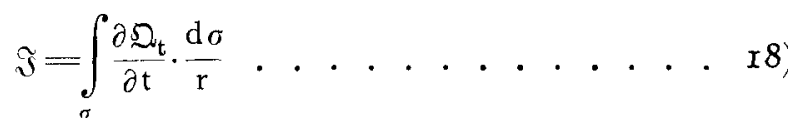

Zur Berechnung der induzierten Feldstärke $\mathfrak{F}_{\mathrm{i}}$ haben wir also die Gleichung:

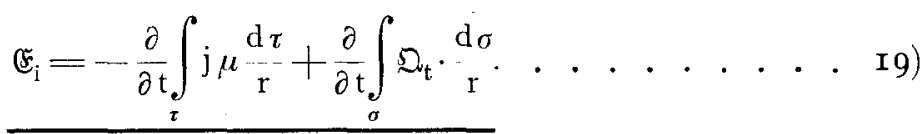

Bei der Anwendung dieses Resultates auf lineare Leiter hat man zwei Fäle zu unterscheiden; nämlich für das Verhalten von $\dot{C}_{\mathrm{i}}$ außerhalb und innerhalb des Leiters. Das erste Integral ergibt für Punkte, die im Außenraum genügend weit vom Leiter entfernt $\operatorname{sind}^{1}$ ):

$$
\left.\frac{\mathrm{d}(\mathrm{i} \mu)}{\mathrm{dt}} \int_{\mathrm{s}} \frac{\mathrm{ds}}{\mathrm{r}} \cdot . \quad . \quad . \quad . \quad . \quad . \quad . \quad . \quad .20\right)
$$

[s = krummlinige Koordinate längs des Leiters].

Für das zweite Integral in I9) ist nach 8), II) und $I 7^{\prime}$ ), wenn $\dot{\tau}_{1}$ den Einheitsvektor in der Stromrichtung bedeutet, zu setzen:

es lautet deshalb:

$$
\Omega_{\mathrm{t}}=\frac{\mu-\mathrm{I}}{4 \pi} \cdot \frac{2 \mathrm{i}}{\varrho} \cdot \mathrm{i}_{1}
$$

$$
\frac{\mathrm{I}}{2 \pi \varrho} \cdot \frac{\operatorname{di}(\mu-I)}{\mathrm{dt}} \cdot \int_{\sigma} \frac{i_{1}}{\mathrm{r}} \mathrm{d} \sigma .
$$

1) Es ist für die Konvergenz des Integrales notwendig, dafi der Quotient, gebildet ans der Leiterdicke $\varrho$ und der Entfernung des Aufpunktes, vom nächsten Leiterpunkt eine verschwindend kleine Gröfe darstellt.

Bolliger, Potentialthcorie. 
und, wenn ds die Rolle eines Differentialvektors übernimmt:

$$
\left.\left.\frac{\mathrm{di}(\mu-\mathrm{I})}{\mathrm{dt}} \cdot \int_{\mathrm{g}} \frac{\mathrm{ds}}{\mathrm{r}} \mathrm{1}\right) . . . . . . .2 \mathrm{I}\right)
$$

Mit Rücksicht darauf, daß es wegen $\operatorname{div} j=0$ nur geschlossene Ströme gibt, folgt durch Zusammenfassung von 20) und 2I) für den Außenraum das Resultat:

$$
\left.\mathfrak{E}_{\mathrm{i}}=-\frac{\mathrm{di}}{\mathrm{dt}} \oint_{\mathrm{g}} \frac{\mathrm{d} \mathrm{s}}{\mathrm{r}} \ldots . . . . . .22\right)
$$

Man ersieht daraus, daß es für den Außenraum ganz einerlei ist, aus was für einem Material der lineare Leiter besteht. Der Betrag der Feldstärke $\mathbb{E}_{\mathrm{i}}$ ergibt sich lediglich aus der Konfiguration des Leiters und aus der zeitlichen Änderung des Stromes. Das Resultat 22) ist auch physikalisch vollständig plausibel. Wie wir nämlich früher, S. I5, gesehen haben, ist die magnetische Feldstärke $\mathfrak{H}$ außerhalb und innerhalb eines linearen Leiters vollständig unabhängig vom Leitermaterial und das Linienintegral der elektrischen Feldstärke hängt im Außenraume für einen beliebigen Integrationsweg wegen $\mu=I$ dort nach der zweiten Maxwellschen Differentialgleichung blo $\mathfrak{B}$ von $\mathfrak{H}$ ab und damit auch die Feldstärke $\mathscr{E}_{i}$.

Bildet man das Linienintegral der elektrischen Feldstärke $\xi_{i}$ über eine Kurve s' durch skalare Multiplikation der Werte von $\xi_{i}$ mit den Kurvenelementen, so erhält man:

$$
\mathrm{e}_{\mathrm{i}}=\int_{\mathbf{g}^{\prime}}\left(\mathfrak{E}_{\mathrm{i}} \mathrm{d} \mathrm{s}^{\prime}\right)=-\frac{\mathrm{di}}{\mathrm{dt}} \int_{\mathrm{s}^{\prime}}\left(\mathrm{d} \mathrm{s} \oint_{\mathrm{s}} \frac{\mathrm{ds}}{\mathrm{r}}\right)=-\frac{\mathrm{di}}{\mathrm{dt}} \int_{\mathbf{s}^{\prime}} \oint_{\mathbf{s}} \frac{\left(\mathrm{ds} \mathrm{s}^{\prime} \mathrm{ds}\right)}{\mathrm{r}}
$$

und wenn $\mathrm{s}^{\prime}$ eine geschlossene Kurve ist:

$$
\mathrm{e}_{\mathrm{i}}=-\frac{\mathrm{di}}{\mathrm{dt}} \oint_{\mathrm{s}} \oint_{\mathrm{s}^{\prime}} \frac{\left(\mathrm{d} \mathrm{s} \mathrm{d} \mathrm{s}^{\prime}\right)}{\mathrm{r}}
$$

Das hier vorkommende Doppelintegral ist das sog. F. Neumannsche Konfigurationsintegral, nach welchem die Induktionskoeffizienten der Leiter berechnet werden. Meistens wird angenommen, daß $s$ und $s^{\prime}$ auch dieselben Kurven sein dürfen und $\mathrm{daB}$ unter dieser Voraussetzung das Neumannsche Integral den sog. Selbstinduktionskoeffizienten dieser Kurve bzw. eines gleichgestalteten linearen Leiters darstelle. Dies ist indessen meiner Beurteilung nach nicht zulässig, weil die Singularität im Integranden von 23 ) bei zusammenfallender Lage der Elemente $\mathrm{ds}$ und $\mathrm{ds}^{\prime}$ [also für $\mathrm{r}=0$ ] stets so hoch ist, daß das Integral unter allen Umständen divergiert. Das Neumannsche Integral trägt der Tatsache nicht Rechnung, daß der Selbstinduk. tionskoeffizient eines Leiters stark von dessen Dicke abhängig ist und im Falle eines unendlich dünnen Leiters überhaupt seinen Sinn verliert.

Die richtige Berechnung der Selbstinduktionskoeffizienten ergibt sich auf Grund der Werte, welche die Formel I9) im Innern von sehr dünnen Leitern für $\bigotimes_{i}$ liefert. Jenes Integral gibt auch sofort Aufschluß darüber, wovon die Singularität im Neumannschen Integral herrührt. Während beim endlich dimensionierten Leiter die durchströmten Teilchen stets so gruppiert sind, daß in nächster Nähe des Aufpunktes für $\mathfrak{F}_{\mathrm{i}}$ auch nur eine verschwindend kleine Strommenge passiert, trifft dies beim linearen Leiter nicht $z u$. Die einzelnen Teilströme sind ohne Reduktion ihrer Wirkung in ihrer ganzen Zahl in den Aufpunkt hineinverlegt, wodurch die Konvergenz des Integrales aufhört. Die richtige Formel läßt sich aber nur dadurch erhalten, daß bei der Zurückführung des

1) Es ist nämlich im vorhergehenden Ausdrucke:

$$
\int_{\sigma} \frac{\mathrm{i}_{1}}{\mathrm{r}} \mathrm{d} \sigma=\int_{\mathrm{s}} \frac{\mathrm{ds}}{\mathrm{r}} \int_{0}^{2 \pi} \varrho \mathrm{d} \alpha=2 \pi \varrho \int_{\mathrm{s}} \frac{\mathrm{ds}}{\mathrm{r}} .
$$


Leiters mit endlichem Querschnitt auf den linearen die ursprüngliche Stromverteilung durch eine solche fingierte [in Wirklichkeit unmögliche, die der Kontinuität des Stromes widerspricht] in der Leiterachse ersetzt wird, daß der nunmehr für den linearen Leiter berechnete Selbstinduktionskoeffizient demjenigen des endlich dimensionierten möglichst nahe kommt.

Um die erwähnte Stromverteilung finden zu können, nehmen wir an, daß der Strom beim räumlichen Leiter sich gleichmäßig über den Querschnitt verteile. Für unsere Untersuchung ist dies berechtigt, weil der Skineffekt erst bei relativ hohen Frequenzen und größeren Leiterdicken, als sie für uns in Frage kommen, in störender Weise bemerkbar wird. Wir berechnen die beiden Integrale in 19) für einen beliebigen Punkt $P$ in der Achse eines Leiters vom Durchmesser $2 \varrho$ [vergleiche dazu Abb. 4], den wir uns zu diesem Zwecke durch sehr viele Normalebenen zur Achse segmentiert denken. Die räumliche Stromverteilung jedes Segmentes ersetzen wir durch eine solche lineare in der Leiterachse, daß deren Beiträge an die Integrale in 19) der ursprünglichen Stromverteilung entsprechen. Unsere Betrachtungen führen zu einfachen Resultaten, wenn wir uns die Näherung gestatten, die aber beim Übergang zu unendlich dünnen Leitern asymptotisch richtig wird, daß alle Punkte eines Segmentes, die den

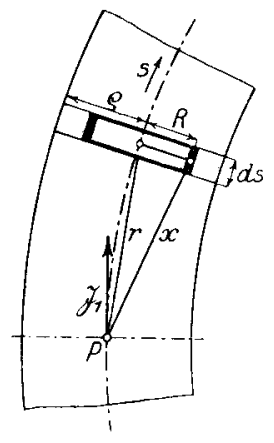

Abb. 4 . gleichen Abstand $\mathrm{R}$ von der Leiterachse besitzen, auch ein und dieselbe Entfernung $\mathrm{x}$ vom Aufpunkte haben. Dann ist nach der Figur:

$$
\begin{aligned}
\mathrm{x} & =\sqrt{\mathrm{r}^{2}+\mathrm{R}^{2}} \\
\mathrm{~d} \tau & =2 \pi \mathrm{RdR} \mathrm{ds} \mid
\end{aligned}
$$

und das crste Integral [Vektor $\mathfrak{J}_{1}$ ] in I9) lautet:

$$
\widetilde{\Im}_{1}=-2 \pi|\mathrm{j}| \mu \oint_{\mathrm{s}} \mathrm{d} \int_{0}^{\rho} \frac{\mathrm{RdR}}{\sqrt{\mathrm{r}^{2}+\mathrm{R}^{2}}},
$$

und da das innere Integral vom Betrage:

und

$$
\sqrt{\mathrm{r}^{2}+\varrho^{2}}-\mathrm{r}=\frac{\varrho^{2}}{\mathrm{r}+\sqrt{\mathrm{r}^{2}+\varrho^{2}}}
$$

ist, folgt:

$$
\mathrm{i}=|\mathrm{j}| \pi \varrho^{2}
$$

$$
\Im_{1}=-2 \mathrm{i} \mu \oint_{\mathrm{r}} \frac{\mathrm{ds}}{\mathrm{r}+\sqrt{\mathrm{r}^{2}}+\overline{\varrho^{2}}} \ldots . . . \text { 24) }^{\prime}
$$

Für die auf den Aufpunkt reduzierte (fingierte) Dichte hat man also den Ausdruck:

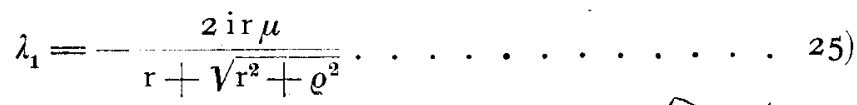

In ganz analoger Weise berechnen wir mit Hilfe von Abb. 5 das zweite Integral von I9) für einen innern Punkt des Leiters. Wir nehmen dazu an, daß alle Flächenelemente an der Umgrenzung eines Leitersegmentes denselben Abstand y vom Aufpunkte der Feldstärke haben. Dann ist:

$$
\begin{aligned}
\mathrm{y} & =\sqrt{\mathrm{r}^{2}+\varrho^{2}}, \\
\mathrm{~d} \sigma & =2 \pi \varrho|\mathrm{ds}|
\end{aligned}
$$

und nach S. 17 muß gesetzt werden:

$$
\mathfrak{D}_{\mathrm{t}}=\frac{\mu-\mathrm{I}}{4 \pi} \cdot{ }_{\varrho}^{2 \mathrm{i}} \mathrm{i}_{1}
$$

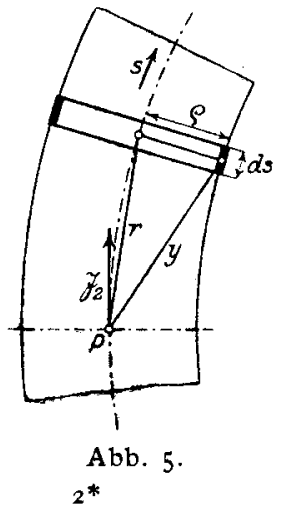


so daß das zweite Integral [Vektor $\mathfrak{\Im}_{2}$ ] in Ig) lautet:

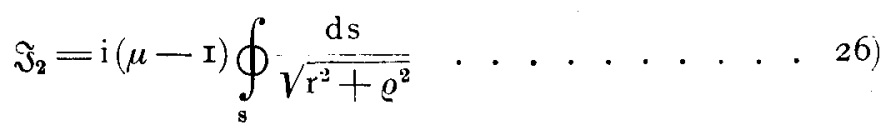

und die diesbezügliche reduzierte lineare Dichte ist:

$$
\left.\lambda_{2}=\frac{\operatorname{ir}(\mu-\mathrm{I})}{\sqrt{\mathrm{r}^{2}+\varrho^{2}}} \text {. . . . . . . . . . . } 27\right)
$$

Die Dichten $\lambda_{1}$ und $\lambda_{2}$ sind also abhängig von der Stromstärke $\mathrm{i}$, der Permeabilität $\mu$ des Drahtes, dann aber hauptsächlich auch von der Drahtdicke $2 \varrho$ und dem Abstande $\mathrm{r}$ des Aufpunktes. Sie haben die Eigenschaft, daß sie in der Gegend des Aufpunktes die räumliche Stromverteilung der Wirklichkeit entsprechend mit asymptotischer Genauigkeit berücksichtigen, weil dort die Konstanz der Abstände $\mathrm{x}$ und y für alle Punkte, welche auf Drehkreisen zur Leiterachse liegen, zutrifft. Am Aufpunkte ist:

$$
\lim _{r=0}\left(\frac{\lambda_{1}+\lambda_{2}}{r}\right)=-\frac{i(\mu+r)}{\varrho}
$$

Man erkennt daraus den schwerwiegenden Einfluß der Permeabilität des Leitermaterials und der Drahtdicke, während für größere Entfernungen $r$ vom Aufpunkte mit der Vernachlässigung von $\varrho$ gegen $r$ näherungsweise gilt:

$$
\frac{\lambda_{1}+\lambda_{2}}{\mathrm{r}} \simeq-\frac{\mathrm{i}}{\mathrm{r}}
$$

d. h. auch in diesem Falle geben die Dichten genähert richtige Werte. Die Ströme erscheinen dafür beinahe als konzentriert, wie es der Auffassung beim Neumannschen Integrale entspricht, und der Einfluß der Permeabilität tritt in den Hintergrund. Außerdem ist zu bemerken, daß beim Übergang zu unendlich kleinen Werten des Drahtdurchmessers $\lambda_{1}$ und $\lambda_{2}$ asymptotisch richtig werden, und damit auch:

d. h.

$$
\mathbb{E}_{\mathrm{i}}=\frac{\partial}{\partial \mathrm{t}} \oint_{\mathrm{s}}\left(\lambda_{1}+\lambda_{\mathrm{a}}\right)_{\mathrm{r}}^{\mathrm{ds}}
$$

$$
\left.\mathbb{E}_{\mathrm{i}}=-2 \frac{\mathrm{d}(\mathrm{i} \mu)}{\mathrm{d} \mathrm{t}} \oint_{\mathrm{s}} \frac{\mathrm{ds}}{\mathrm{r}+\sqrt{\mathrm{r}^{2}}+\varrho^{2}}+\frac{\mathrm{di}(\mu-\mathrm{r})}{\mathrm{dt}} \oint_{\mathrm{B}} \frac{\mathrm{ds}}{\sqrt{\mathrm{r}^{2}}+\varrho^{2}} \ldots .28\right)
$$

$\varepsilon_{i}$ ist für $\varrho=0$ selber unendlich, wird aber für hinlänglich dünne Drähte durch 28) sehr genau ausgedrückt.

Die induzierte Spannung $\mathrm{e}_{\mathrm{i}}$ längs der Drahtachse ist numerisch gleich dem diesbezüglichen Linienintegral der elektrischen Feldstärke $\mathfrak{F}_{\mathrm{i}}$, wird also in analoger Weise erhalten wie auf S. I8. Ich gelange so zu der für $\varrho=0$ asymptotischen Darstellung:

$$
\mathrm{e}_{\mathrm{i}}=-2 \frac{\mathrm{d}(\mathrm{i} \mu)}{\mathrm{d} \mathrm{t}} \oint_{(\mathbf{s})} \oint_{\mathrm{r}+\sqrt{\mathrm{r}^{2}+\varrho^{2}}}+\frac{\mathrm{di}(\mu-\mathrm{I})}{\mathrm{dt}} \oint_{(\mathrm{s})} \oint_{\frac{\left(\mathrm{d} \mathrm{s} \mathrm{ds}^{\prime}\right)}{\sqrt{\mathrm{r}^{2}+\varrho^{2}}}} . .2 \text {. 29) }
$$

[die Integrationen der innern und äußeren Integrale erstrecken sich über dieselbe Kurve s].

Im Falle eines nichtmagnetisierbaren Leiters ist:

$$
\mathrm{e}_{\mathrm{i}}=-\ldots \mathrm{L} \frac{\mathrm{di}}{\mathrm{dt}}
$$

es wird daher der Selbstinduktionskoeffizient L nach 29) mit Berücksichtigung des Drahtdurchmessers asymptotisch folgendermaßen dargestellt: 


$$
\begin{aligned}
& -2 I_{-}^{-} \\
& \mathrm{L}=2 \oint_{(\mathrm{s})} \oint_{\left.\mathrm{r}+{\sqrt{\mathrm{r}^{2}+\varrho^{2}}}^{1}\right)}^{\left(\mathrm{dsd}^{\prime}\right)} . . . . . .30
\end{aligned}
$$

[r hat hierin die gleiche Bedeutung wie im Neumannschen Integrale].

Die vollständige Behandlung unserer Aufgabe über die linearen Leiter benötigt noch die Integration von Gl. I6), welche lautet:

$$
\nabla^{2} \mathfrak{E}_{\varphi}-\operatorname{grad} \operatorname{div} \mathfrak{E}_{\varphi}=\mathbf{0} .
$$

Man kann zeigen, daß der Feldvektor $\mathfrak{E}_{p}$ ein Potential $\varphi$ im Sinne der Elektrostatik hat. Danach müßte sein:

$$
\mathfrak{E}_{q}=-\operatorname{grad} \varphi \text {. . . . . . . . . . . . . 3I) }
$$

Die Verifikation ergibt sich durch Einführung von $3 \mathrm{I}$ ) in die vorige Gleichung, aus der so die gültige Vektorformel hervorgeht:

$$
\left.\nabla^{2} \operatorname{grad} \varphi=\operatorname{grad} \Delta \varphi^{2}\right), \ldots . . . . . . . .
$$

worin $\Delta \varphi$ den Laplaceschen Operator bedeutet.

Da wir im Leiteraußenraum (Vakuum) keine freie Elektrizität angenommen haben, liegt das Quellgebiet des Potentials $\varphi$ einzig im Leiter. Zur Formulierung der Randbedingungen von $\varphi$ gehen wir aus vom Strömungszustand, den wir für einen sehr dünnen Leiter als bekannt voraussetzen dürfen. Aus div $j=0$ folgt nämlich die Kontinuität des Stromes $\mathrm{i}$ und daraus für einen Draht von durchweg gleichem, kreisförmigem Querschnitt $\mathrm{f}$ und homogenem Material [Leitfähigkeit $\lambda=$ konst.] bei gleichmäßiger Verteilung des Stromes über denselben die Konstanz von $j$ längs des Leiters. Wegen $j=\lambda \notin$ ist dann, wenn der Leiter nur hinlänglich dünn ist, das Feld so gut wie homogen und die \&-Linien fallen mit den Stromlinien zusammen. An der Leiteroberfläche ist, wie mit dem Gaußschen Satze aus $\operatorname{div} j=0$ folgt, $j_{n}=0$; d. h. die Stromlinien fallen dort in die Grenzfläche, und die Niveauflächen des Potentials $\varphi$ bilden Normalschnitte zur Strombahn.

Da der Strom im ganzen Kreise nach einerlei Richtung fließt, fällt $\varphi$ in dieser Richtung fortwährend und das Linienintegral von $F_{\varphi}$ kann nicht verschwinden. Es muß also irgendwo eine Diskontinuitätsstelle des Potentials geben. Eine solche kann erfahrungsgemä $\beta$ hervorgerufen werden durch kontakt-elektromotorische Kräfte, galvanische Elemente oder eingeprägte induzierte Spannungen. Bezeichnen $\varphi_{1}$ und $\varphi_{2}$ die Potentialwerte von $\varphi$ zu beiden Seiten der Unstetigkeitsstelle, so folgt für die längs der ganzen 'Strombahn konstante Feldstärke $\mathfrak{E}_{\varphi}$ nach $3 \mathrm{I}$ ):

$$
\begin{gathered}
\mathfrak{E}_{f}=\frac{\varphi_{1}-\varphi_{2}}{\mathrm{l}}=\mathrm{e}_{\varphi}, \\
{[\mathrm{l}=\text { Länge der Strombahn }]}
\end{gathered}
$$

und im Leiter gilt also als Integralgesetz von

$$
\mathrm{j}=\lambda \stackrel{\xi}{E}
$$

1) Von der nicht vollständig exakten, sondern blof asymptotischen Darstellung abgesehen, wird Formel 30) allerdings den physikalischen Verhältnissen noch nicht in jeder Beziehung gerecht, weil der Skineffekt nicht in Rücksicht gezogen worden ist. In Wirklichkeit sind die in Parallelkurven zur Drahtachse induzierten Spannungen von der berechneten etwas verschieden. Doch ist nach den bereits oben angegebenen Gründen der Fehler sehr klein.

2) Es ist nämlich für die $x$-Komponente:

$$
-\frac{\partial^{2}\left(\frac{\partial \varphi}{\partial \mathbf{x}}\right)}{\partial \mathbf{x}^{2}}+\frac{\partial^{2}\left(\frac{\partial \varphi}{\partial \mathbf{x}}\right)}{\partial \mathbf{y}^{2}}+\frac{\partial^{2}\left(\frac{\partial \varphi}{\partial \mathbf{x}}\right)}{\partial \mathbf{z}^{2}} \equiv \frac{\partial}{\partial \mathbf{x}}\left\{\frac{\partial^{2} \varphi}{\partial \mathbf{x}^{2}}+\frac{\partial^{2} \varphi}{\partial \mathbf{y}^{2}}+\frac{\partial^{2} \varphi}{\partial \mathbf{z}^{2}}\right\},
$$

und Analoges gilt für die andern Komponenten. 
das Ohmsche Gesetz:

in welchem

$$
\mathrm{i}=\frac{\mathrm{e}_{p}}{\mathrm{w}}, \ldots . \ldots . . . . . .
$$

$$
\mathrm{w}=\frac{\mathrm{l}}{\lambda \cdot \mathrm{f}}
$$

den sog. Leitungswiderstand bedeutet. Der Übergang von einem räumlichen Leiter zu einem linearen als Ersatz muß nun logischer Weise stets so erfolgen, daß der Strom i und die Spannungsdifferenz $e_{\varphi}$ dabei ungeändert bleiben. Nach dem Ohmschen Gesetze muB deshalb der Widerstand $w$ und danach also das Produkt $\lambda$. $f$ konstant gehalten werden. $\lambda$ wird daher beim Grenzübergang unendlich.

Nach unseren Betrachtungen ist bei gegebener Spannungsdifferenz $\mathrm{e}_{\varphi}=\varphi_{1}-\varphi_{2}$ nach dem Ohmschen Gesetze im Leiterinnern die Feldstärke $\tilde{E}_{\varphi}$ vollständig; hingegen das Potential $\varphi$ nur bis auf eine additive Konstante $\Phi$ bestimmt. Diese Konstante $\Phi$ hat offenbar auf den Strömungszustand keinen Einfluß; sie ist vollständig willkürlich. So kann man ohne Nachteil irgendeinen Punkt des Leiters erden oder künstlich auf ein beliebiges Potential bringen.

Soll das Potential $\varphi$ aber auch für den Leiteraußenraum ermittelt werden, so ist die Angabe dieser Konstanten $\Phi$ bzw. der absoluten Potentialwerte unerläBlich; denn nach einem bekannten Satze der Potentialtheorie ${ }^{1}$ ) ist das statische Potential in einem Raume dann und nur dann eindeutig bestimmt, wenn die Randwerte von $\varphi$ an der ganzen Begrenzung dieses Raumes gegeben sind. $\mathrm{Zu}$ der Begrenzung unseres Außenraumes (des die Leiter umgebenden Vakuums) zählt aber auch die unendlich ferne Kugel, wo $\varphi$ verschwindet; es ist daher gar nicht einerlei, welchen Wert $\Phi$ hat.

Für den Leiteraußenraum ist nun $\varphi$ nach I6) bezw. 32) der Gleichung:

$$
\Delta \varphi=\text { o . . . . . . . . . . . 34) }
$$

entsprechend so zu bestimmen, daß die Randwerte an der Leiteroberfläche mit denjenigen des Leiterinnern übereinstimmen und daß $\varphi$ im Unendlichen verschwindet. $\varphi$ ist im Außenraum darstellbar als eine Potentialfunktion von Massenverteilungen, welche teils im Leiter und teils auf dessen Oberfläche verteilt sind Für die Be. stimmung von $\varphi$ kommt es also lediglich darauf an, diese Massenverteilung zu finden. Die vorliegende Aufgabe wird für beliebige Randwerte eines räumlichen Leiters allgemein gelöst durch die Hilfsmittel, welche in jüngster Zeit die Theorie der Fredholmschen Integralgleichung geschaffen hat. Da jedoch die auf diesem Wege gewonnenen, sehr allgemeinen Resultate für die praktische Anwendung unbrauchbar sind, schlage ich hier einen andern Weg ein, der uns allerdings für einen räumlichen Leiter nicht die exakte Lösung, hingegen numerisch verwertbare Näherungsresultate zu liefern imstande ist. Wir denken uns zu diesem Zwecke den endlichen Leiter durch einen unendlich dünnen ersetzt.

An einer linearen Massenverteilung wird $\varphi$ unendlich und läßt sich in unmittelbarer Nähe derselben darstellen als das Produkt $\Pi$ einer Funktion $\Lambda$, die nur von der Längskoordinate der Massenverteilung und einer Funktion $\Gamma$, die nur vom Abstande des Aufpunktes vom nächsten Massenpunkte abhängig ist. $\varphi$ ist an der Grenze also von der Form:

$$
\Pi=\Lambda \cdot \Gamma \text {. . . . . . . . . . . . . . } 35
$$

1) Siehe C. Neumann, Untersuchungen iber das Newtonsche und logarithmische Potential, S. 4I, wo folgender Satz bewiesen wird: Bezeichnet $\phi$ das Potential irgendwelcher unbekannter, auserhalb des Gebietes $\tau$ oder auf seiner Grenze gelegener Massen, so ist dieses Potential $\varphi$ fur alle Punkte innerbalb $\tau$ völlig bestimmt, sobald nur seine Werte auf der Grenze von $\tau$ gegeben sind. 
Wir lösen nun die Aufgabe:

Es soll ein Potential $\varphi$ bestimmt werden, für welches die Randwerte längs einer. stetigen, aber sonst beliebigen, linearen Massenverteilung durch die Funktion $A$ willkiurlich vorgegeben sind.

$\mathrm{Zu}$ diesem $Z$ wecke berechnen wir das Potential irgendeiner linearen Verteilung für einen Punkt $P$ in der Mittelnormalebene des Linienelementes $(-\mathrm{h},+\mathrm{h})$ mit der Liniendichte [Elektrizitätsmenge pro Längeneinheit] $\lambda$ [vgl. Abb. 6].

Es ist:

$$
\underset{(-\mathbf{h},+\mathbf{h})}{\varphi=\lambda} \int_{-\mathbf{h}}^{+\mathrm{d}} \frac{\mathrm{ds}}{\mathrm{r}}=\lambda \lg \frac{\sqrt{\underline{Q}^{2}+\mathrm{h}^{2}}+\mathrm{h}}{\sqrt{\underline{Q}^{2}+\mathrm{h}^{2}}-\mathrm{h}}
$$

oder:

$$
=2 \lambda\left[\lg \frac{\mathrm{I}}{\varrho}+\lg \left(\sqrt{\varrho^{2}+\mathrm{h}^{2}}+\mathrm{h}\right)\right] \text {. }
$$

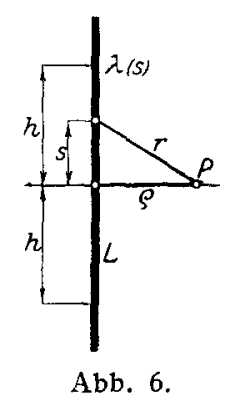

Der Beitrag der übrigen Massen ist eine für den Punkt $\mathrm{P}$ reguläre Funktion; daher gilt im Grenzwerte $\varrho=0$ :

$$
\Pi=2 \lambda \cdot \lg \frac{\mathrm{I}}{\varrho}+\text { f. c. . . . . . . . . . . } 36
$$

und nach 35) kann gesetzt werden:

$$
\left.\begin{array}{l}
A=2 \lambda \\
\Gamma=\lg \frac{\mathrm{I}}{\varrho}
\end{array}\right\}
$$

und es resultiert daraus der Satz:

Satz: An einer stetigen linearen Massenverteilung ist das Potential proportional der linearen Dichte; der Proportionalitätsfaktor ist be. stimmt durch den Logarithmus der kürzesten Entfernung des Aufpunktes von der Achse der Verteilung ${ }^{\text {) }}$.

Damit ist aber die oben gestellte Aufgabe bereits gelöst. Man braucht, um das Potential $\varphi$ mit dem Randwerte $A \Gamma$ zu finden, bloß die Liniendichte $\lambda$ Gl. 37) entsprechend anzunehmen.

Die Anwendung des gefundenen Resultates auf den Fall von einem endlichen, aber noch hinreichend dünnen Leiter bei dem vorher behandelten Strömungsprobleme basiert darauf, daß die in 36) vorkommende functio continua bei nicht melir verschwindendem, aber doch noch genügend kleinem $\varrho$ praktisch vernachlässigt werden darf.

Für einen räumlichen Leiter, bei welchem die Randwerte $I I$ des Potentials $\varphi$ aus dem Strömungszustande im Leiter bekannt sind, berechnet sich dann das Potential des Außenraumes näherungsweise durch Quadratur über die folgende als Ersatz für die wirkliche Raumdichte dienende lineare Dichte in der Leiterachse:

$$
\lambda=\frac{I I}{2 \lg \frac{\mathrm{I}}{\varrho}} \cdot \ldots . . . . . . . . .
$$

1) Furr irgend zwei Punkte eines statisch gcladenen, linearen Leiters, bei welchem der infiniesimale Durchmesser eine stetige Funktion der Längskoordinate ist, gilt nach 36 ):

oder also:

$$
\lambda_{1} \lg \frac{1}{\varrho_{1}}=\lambda_{2} \lg \frac{1}{\varrho_{2}}
$$

$$
\underline{\underline{0}_{1}{ }^{\lambda_{1}}={\underline{Q_{2}}}^{\lambda_{2}}} \text {. }
$$

Ist speziell die Leiterdicke überall gleich, so muß auch die Liniendichte längs des ganzen Leiters dieselbe sein. 
Für ein homogenes Leitermaterial ist $I I$ und damit auch $\lambda$ bei überall gleichem $\varrho$ ,nach dem Ohmschen Gesetze eine lineare Funktion der Längskoordinate des Leiters.

Unsere Untersuchung über die linearen Leiter erübrigt noch festzustellen, inwieweit der Einfluß der vom Durchfluß des Stromes hervorgerufenen (scheinbar statischen) Ladung des Leiters $z u$ berïcksichtigen ist. Der raschen Fortpflanzung der Elektrizität und der Kontinuität des Stromes wegen muß die Elektronendichte in einem Leiter von konstantem Querschnitte überall gleich sein. Der durch den Strom mitgeführten Elektrizität entspricht also ein ,statisches“ Potential mit konstanter linearer Dichte des Leiters. Nach 36) besitzt es also, genau so, wie es bei einem statisch geladenen Konduktor der Fall wäre, einen konstanten Randwert. Da aber solche konstante Leiterpotentiale bereits in der auf S. 22 eingeführten Konstanten $\Phi$ miteinbezogen sind, braucht das hier besprochene Potential überhaupt nicht mehr besonders berücksichtigt zu werden.

\section{Induzierte Spannung und asymptotischer Selbstinduktionskoeffizient des Kreisleiters.}

Wir berechnen zuerst die auf S. 20 formulierten Integrale der Feldstärke $\mathfrak{E}_{i}$ für einen Punkt $P$ in der Achse des Kreisleiters [Kreisdurchmesser $=R$, Drahtdurchmesser $=2 \varrho]$. Nach S. 20, Formel 28) gilt:

$$
\left.\Sigma_{i}=-2 \frac{\mathrm{d}(\mathrm{i} \mu)}{\mathrm{dt}} \oint_{\mathrm{s}} \frac{\mathrm{ds}}{\mathrm{r}+\sqrt{\mathrm{r}^{2}+\varrho^{2}}}+\frac{\mathrm{di}(\mu-I)}{\mathrm{dt}} \oint_{\mathrm{s}} \frac{\mathrm{ds}}{\sqrt{\mathrm{r}^{2}+\varrho^{2}}} \ldots . . . \mathrm{I}\right)
$$

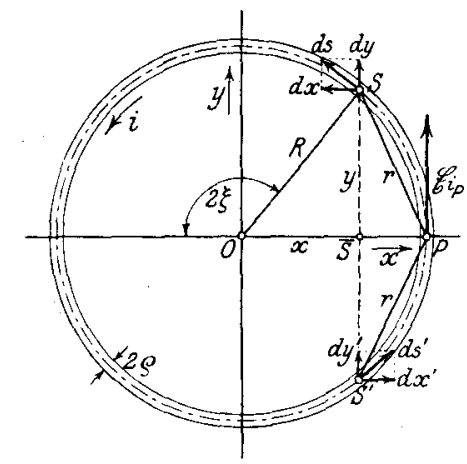

Abb. 7 .

Die Integrale sind zu erstrecken über die einzelnen Komponenten der Linienelemente ds der Kreisbahn.

Aus Abb. 7 geht hervor, daß die $\mathrm{x}$-Komponente von $\tilde{E}_{\mathrm{i}, \mathrm{p}}$ verschwindet; denn es heben sich die Differentialbeiträge der in $\mathbf{x}$ ) vorkommenden Integrale für die nach der $\mathrm{x}$-Richtung genommenen Komponenten von symmetrisch zur $\mathrm{x}$-Achse gelegenen Elementen ds und $\mathrm{ds}^{\prime}$ gegenseitig auf. Anders verhält es sich jedoch bei der Integration über die $\mathrm{y}$-Komponenten der Differentialvektoren. Symmetrisch zur $\mathrm{x}$-Achse gelegene Elemente $d s$ und $d s^{\prime}$ liefern gleiche Beiträge an das hierfür in Frage kommende Integral.

Nach I) sind die folgenden Integrale zu berechnen:

und

$$
\left.\mathfrak{V}_{\mathbf{1}}=\oint \frac{\mathrm{dy}}{\mathrm{r}+\sqrt{\mathrm{r}^{2}}+\bar{\varrho}^{2}}=\frac{\mathrm{r}}{\varrho^{2}}\left\{\oint \sqrt{\mathrm{r}^{2}}+\overline{\varrho^{2}} \cdot \mathrm{dy}-\oint \mathrm{rdy}\right\} . . . .2\right)
$$

$$
\left.\mathfrak{Y}_{2}=\oint \frac{\mathrm{dy}}{\sqrt{\mathrm{r}^{2}+\varrho^{2}}}, \ldots . . . . \cdot 3\right)
$$

worin nach Abb. 7 für $\mathrm{r}^{2}$ zu setzen ist:

$$
\left.\mathrm{r}^{2}=(\mathrm{R}-\mathrm{x})^{2}+\mathrm{y}^{2}=2 \mathrm{R}(\mathrm{R}-\mathrm{x})=2 \mathrm{R}\left(\mathrm{R}-\sqrt{\mathrm{R}^{2}-\mathrm{y}^{2}}\right) . . . \quad 4\right)
$$

Die Integrale in 2) sind demnach von der Form

$$
\left.\oint \sqrt{\mathrm{p}-\sqrt{\mathrm{R}^{2}-\mathrm{y}^{2}}} \cdot d y \ldots . . . . .5\right)
$$

[p bedeutet eine Konstante]

und also von elliptischem Charakter. Gl. 5) wird vermöge der Substitution

$$
\mathrm{y}=\mathrm{R} \sin (\pi-2 \xi) \text {. . . . . . . . . 6) }
$$


mit Berücksichtigung der Symmetrie der Stromverteilung in bezug auf die x-Achse übergeführt in:

$$
\left.\frac{4 R \sqrt{2 R}}{k} \int_{0}^{\frac{\pi}{2}}\left(I-2 \sin ^{2} \xi\right) \sqrt{I-k^{2} \sin ^{2} \xi} \cdot d \xi, \quad . . . . .77\right)
$$

wobei der Modul $\mathrm{k}$ vom Betrage ist:

$$
\left.\mathrm{k}=\sqrt{\frac{2 \mathrm{R}}{\mathrm{p}+\mathrm{R}}} \cdot \cdot \cdot \cdot \cdot \cdot \cdot \cdot \cdot \cdot \cdot \cdot \cdot 8\right)
$$

Um das Integral 7) den numerischen Rechnungen zugänglich zu machen, muß der Term

$$
\left.\int_{0}^{\frac{\pi}{2}} \sin ^{2} \xi \sqrt{\mathrm{I}-\mathrm{k}^{2} \sin ^{2} \xi} \cdot \mathrm{d} \xi \quad . \quad . \quad . \quad . \quad . \quad . \quad . \quad . \quad .99\right)
$$

noch durch normalelliptische Integrale ausgedrückt werden.

Mit Einführung der Bezeichnung

$$
\Xi=\sqrt{\mathrm{I}-\mathrm{k}^{2} \sin ^{2} \xi} \text {. . . . . . . . . . . . Io) }
$$

formen wir 9) auf zwei Arten um. Durch Erweiterung des Integranden mit $\Xi$ folgt:

$$
\left.\int \sin ^{2} \xi \Xi \mathrm{d} \xi=\int \frac{\sin ^{2} \xi}{\Xi} \mathrm{d} \xi-\mathrm{k}^{2} \int \frac{\sin ^{4} \xi}{\Xi} \mathrm{d} \xi . . . . . . \mathrm{II}\right)
$$

Ferner geht aus 9) vermittels partieller Integration hervor:

$$
2 \int \sin ^{2} \xi \Xi \mathrm{d} \xi=-\sin \xi \cos \xi \Xi+\int \Xi \mathrm{d} \xi-\mathrm{k}^{2} \int \frac{\sin ^{2} \xi}{\Xi} \mathrm{d} \xi+\mathrm{k}^{2} \int \frac{\sin ^{4} \xi}{\Xi} \mathrm{d} \xi . . \quad \text {. } 2 \text { 2) }
$$

Durch Addition von II) und I2) resultiert nun:

Da aber

$$
\left.3 \int \sin ^{2} \xi \Xi \mathrm{d} \xi=-\sin \xi \cos \xi \Xi+\int \Xi \mathrm{d} \xi+\left(\mathrm{I}-\mathrm{k}^{2}\right) \int \frac{\sin ^{2} \xi}{\Xi} \mathrm{d} \xi . . . . . \mathrm{I} 3\right)
$$

$$
\left.\int \frac{\sin ^{2} \xi}{\Xi} \mathrm{d} \xi=\frac{\mathrm{I}}{\mathrm{k}^{2}}\left\{\int \frac{\mathrm{d} \xi}{\Xi}-\int \Xi \mathrm{d} \xi\right\} \quad . . . . . . \mathrm{I}_{4}\right)
$$

ist, ergibt sich aus I3) die Transformationsformel:

$$
\int \sin ^{2} \xi \Xi \mathrm{d} \xi=\frac{\mathrm{I}}{3}\left\{\frac{\mathrm{r}-\mathrm{k}^{2}}{\mathrm{k}^{2}} \int \frac{\mathrm{d} \xi}{\Xi}-\frac{\mathrm{I}-2 \mathrm{k}^{2}}{\mathrm{k}^{2}} \int \Xi \mathrm{d} \xi-\sin \xi \cos \xi \Xi\right\} . . \text {. I5) }
$$

Bei der Integration in den Grenzen $0 \div \frac{\pi}{2}$ gibt der letzte Summand in der Klammer rechts keinen Beitrag, und es bleibt bloß eine lineare Kombination der elliptischen Integrale erster und zweiter Gattung. Addiert man zur verdoppelten Gl. I5) noch das restierende elliptische Vollintegral zweiter Gattung von 7) und berücksichtigt die dort vorkommenden konstanten Faktoren, so erhält man:

$$
\left.-\frac{4}{3} \cdot \frac{\mathrm{R} \sqrt{2 \mathrm{R}}}{\mathrm{k}^{3}}\left\{2\left(\mathrm{I}-\mathrm{k}^{2}\right) \int_{0}^{\frac{\pi}{2}} \frac{\mathrm{d} \xi}{\Xi}-\left(2-\mathrm{k}^{2}\right) \int_{0}^{\frac{\pi}{2}} \Xi \mathrm{d} \xi\right\} . . . . . . . \mathrm{I} 6\right)
$$

Im ersten Integrale von 2 ) ist für $\mathrm{p} z \mathrm{u}$ setzen:

und im zweiten:

$$
\left.\begin{array}{l}
\mathrm{p}=\frac{\varrho^{2}+2 \mathrm{R}^{2}}{2 \mathrm{R}} \\
\overline{\mathrm{p}}=\mathrm{R}
\end{array}\right\} \cdot . \cdot . \cdot . \cdot . \cdot . . . \quad . \quad \mathrm{I} 7
$$


Die Moduls in r6) sind deshalb nach 8):

und

$$
\left.\left.\begin{array}{l}
\mathrm{k}=\sqrt{\frac{4 \mathrm{R}^{2}}{4 \mathrm{R}^{2}+\varrho^{2}}} \\
\overline{\mathrm{k}}=\mathrm{I}
\end{array}\right\} . . . . . . \mathrm{I} 8\right)
$$

Mit Rücksicht auf 5) erhält man deshalb aus 2) die Lösung für $\mathfrak{Y}_{1}$ durch normalelliptische Integrale zu:

worin

$$
\left.\left.\mathfrak{Y}_{1}=\frac{8}{3}\left(\frac{\mathrm{R}}{\varrho}\right)^{2}\left[\frac{2-\mathrm{k}^{2}}{\mathrm{k}^{3}} \int_{0}^{\frac{\pi}{2}} \Xi \mathrm{d} \xi-\frac{2\left(\mathrm{I}-\mathrm{k}^{2}\right)}{\mathrm{k}^{3}} \int_{0}^{\frac{\pi}{2}} \frac{\mathrm{d} \xi}{\Xi}-\mathbf{I}\right] 1\right), \ldots . . . \mathrm{r} 9\right)
$$

Mit 4) lautet $\mathfrak{Y}_{2}$ in 3 ):

$$
\Xi=\sqrt{\mathrm{I}-\mathrm{k}^{2} \sin ^{2} \xi} ; \quad \mathrm{k}=-\sqrt{\frac{4 \mathrm{R}^{2}}{4 \mathrm{R}^{2}+\varrho^{2}}} .
$$

$$
\left.\mathfrak{V}_{2}=\frac{\mathrm{I}}{\sqrt{2 \mathrm{R}}} \oint \frac{\mathrm{dy}}{\sqrt{\mathrm{p}-\sqrt{\mathrm{R}^{2}-\mathrm{y}^{2}}}}, \ldots . . .20\right)
$$

wobei $p$ vom Betrage I7) ist. Gl. 20) wird durch die Substitutionen 6) und 8) übergeführt in:

$$
2 \mathrm{k} \int_{0}^{\frac{\pi}{2}} \frac{\mathrm{I}-2 \sin ^{2} \xi}{\sqrt{\mathrm{I}-\mathrm{k}^{2} \sin ^{2} \xi}} \mathrm{d} \xi \ldots . . \ldots . . . .2 \mathrm{.} \text {. . }
$$

Unter Verwendung von Bezeichnung Io) und Berücksichtigung der Gl. I4) folgt daraus:

und hieraus:

$$
2 \mathrm{k}\left[\int_{0}^{\frac{\pi}{2}} \frac{\mathrm{d} \xi}{\Xi}-\frac{2}{\mathrm{k}^{2}}\left(\int_{0}^{\frac{\pi}{2}} \frac{\mathrm{d} \xi}{\Xi}-\int_{0}^{\frac{\pi}{2}} \Xi \mathrm{d} \xi\right)\right]
$$

$$
\left.\mathfrak{Y}_{2}=\frac{2}{\mathrm{k}}\left[2 \int_{0}^{\frac{\pi}{2}} \Xi \mathrm{d} \xi-\left(2-\mathrm{k}^{2}\right) \int_{0}^{\frac{\pi}{2}} \frac{\mathrm{d} \xi}{\Xi}\right] \ldots . . . .22\right)
$$

$\Xi$ und $\mathrm{k}$ sind von der gleichen Bedeutung wie in I9). Durch 22) wird $\mathfrak{9}_{2}$ dargestellt durch elliptische Vollintegrale erster und zweiter Gattung.

Nach I), 2) und 3) ist die längs des ganzen Kreisringes tangential gerichtete und überall gleich große induzierte Feldstärke $\rightleftarrows_{\mathrm{i}}$ gleich:

Die induzierte Spannung ist:

$$
\left.E_{i}=-2 \frac{\mathrm{d}(\mathrm{i} \mu)}{\mathrm{dt}} \mathfrak{V}_{1}+\frac{\mathrm{di}(\mu-\mathrm{I})}{\mathrm{dt}} \mathfrak{V}_{2} . \ldots . . . .23\right)
$$

$$
\left.\mathrm{e}_{\mathrm{i}}=\int_{(\text {Kreis })}\left(\Theta_{\mathrm{i}} \mathrm{ds}\right)=2 \pi \mathrm{R} E_{\mathrm{i}} . . . . . . . .24\right)
$$

Den asymptotischen Selbstinduktionskoeffizienten erhält man aus

$$
\mathrm{e}_{\mathrm{i}}=-\mathrm{L} \frac{\mathrm{di}}{\mathrm{dt}},
$$

wenn man die Permeabilität $\mu$ des Leitermaterials gleich I setzt zu:

$$
\mathrm{L}=4 \pi \mathrm{R} \mathrm{g}_{1} \text {.......... . 25) }
$$

1) Tabellen für die elliptischen Vollintegrale enthält z. B. das Tafelwerk: Funktionentafeln von Jahnke und Emde, Verlag Teubner, Leipzig 1909, S. 54-64. 


\section{Anwendungsbeispiele für das elektrische Potential linearer Leiter.}

\section{A. Stab - Ring - Potential.}

Es soll das elektrostatische Potential eines geraden, linearen Leiterstabes [Länge $=2 \mathrm{~h}$ ] und eines zum Ringe gebogenen linearen Drahtes [Ringdurchmesser $=2 \mathrm{R}$ ] unter der Voraussetzung, daß die Totalelektrizität des Leitersystems Null ist, ermittelt werden. Nach unseren Betrachtungen auf den Seiten 22 bis 24 sind die Liniendichten von elektrostatischen Verteilungen längs linearen Leitern konstant und also direkt durch die Gesamtmassen der einzelnen Leiter ausdrückbar. Die Einzelpotentiale der linearen Leiter sind demnach unabhängig von der relativen Lage der letzteren und das resultierende Potential ergibt sich daraus durch Superposition.

Wir ermitteln daher einzeln die Potentialfunktionen für das Stäbchen und den Ring. Ist die Gesamtmasse am Stäbchen $+\mathrm{m}$ und diejenige am Ringe $-\mathrm{m}$, so sind die Liniendichten:

$$
\begin{aligned}
& \sigma_{1}=+\frac{\mathrm{m}}{2 \mathrm{~h}} \text { am Stabe........... I) } \\
& \sigma_{2}=-\frac{\mathrm{m}}{2 \pi \mathrm{R}} \text { am Ringe.......... . 2) }
\end{aligned}
$$

Bei Verwendung von Zylinderkoordinaten ( $r, z)$ [z-Achse $=$ Stabachse, Koordinatenursprung im Stabhalbierungspunkt] lautet das Potential $\varphi_{1}$ des Stäbchens:

woraus nach Integration folgt:

$$
\left.\varphi_{1}=\frac{\mathrm{m}}{2 \mathrm{~h}} \int_{-\mathrm{h}}^{+\mathrm{h}} \frac{\mathrm{ds}}{\sqrt{\mathrm{r}^{2}+(\mathrm{s}-\mathrm{z})^{2}}}, \ldots . . . . . .33\right)
$$

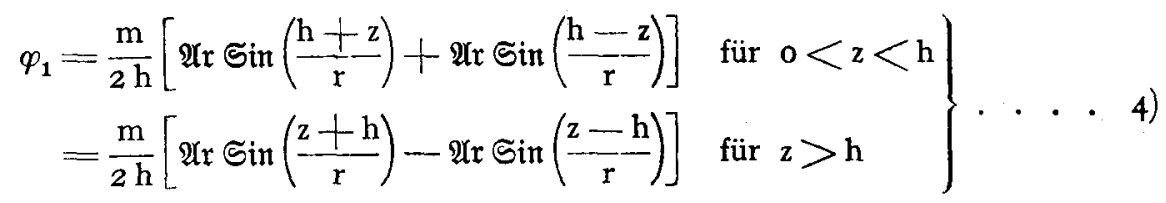

Für negative $z$ ergibt sich das Potential $\varphi_{1}$ aus der Beziehung:

$$
\varphi_{1}(-\mathrm{z})=\varphi_{1}(+\mathrm{z})
$$

Die Potentialfunktion $\varphi_{2}$ des Ringes berechnen wir mit Hilfe von Abb. 8, indem wir ebenfalls Zylinderkoordinaten $(r, z)$ einführen. Der Ring soll in der Ebene $(z=0)$ mit dem Mittelpunkt im Koordinatenanfangspunkt liegen. Ist dann $P^{\prime}$ die Normalprojektion des

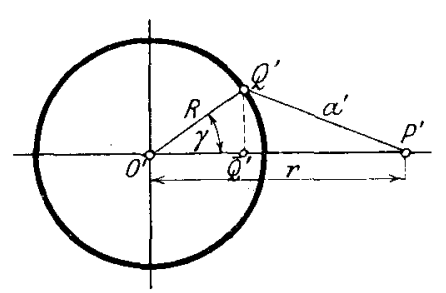

Abb. 8. Aufpunktes $P$ auf die Ebene $(z=0)$, so ist nach der Abbildung:

$$
a=\sqrt{a^{\prime 2}+z^{2}}=\sqrt{R^{2}+r^{2}+z^{2}-2} \overline{R r \cos \gamma} .
$$

Setzt man zur Abkürzung

$$
\mathrm{R}^{2}+\mathrm{r}^{2}+\mathrm{z}^{2}=\mathrm{e}^{2} ; \quad \frac{2 \mathrm{Rr}}{\mathrm{e}^{2}}=\varepsilon, \quad . . . . . .5 \text {. . 5) }
$$

so folgt mit 2) und wegen der Symmetrie des Potentials $\varphi_{2}$ in bezug auf die Meridianebene des Punktes $\mathrm{P}$ für $\varphi_{2}$ das elliptische Integral:

$$
\varphi_{2}=-\frac{\mathrm{m}}{\pi \mathrm{e}} \int_{0}^{\pi} \frac{\mathrm{d} \gamma}{\sqrt{\mathrm{I}-\varepsilon \cos \gamma}}, \ldots . . . . .6
$$


das mit Hilfe der Substitutionen

$$
\varepsilon=\frac{\mathrm{k}^{2}}{2-\mathrm{k}^{2}} ; \quad \gamma=\pi-2 \xi
$$

auf Normalform transformiert werden kann. Es folgt dann aus 6) das elliptische Vollintegral erster Gattung:

$$
\frac{\varphi_{2}=-\frac{2}{\pi} \cdot \frac{\mathrm{m}}{\sqrt{(\mathrm{R}+\mathrm{r})^{2}+\mathrm{z}^{2}}} \int_{0}^{\frac{\pi}{2}} \frac{\sqrt{\mathrm{I}}}{\mathrm{k}}=\sqrt{\frac{4 \mathrm{Rr}}{(\bar{R}+\mathrm{r})^{2}+\mathrm{z}^{2}}}}{}
$$

der an der Grenze $z=0$ für $r=R$ Eins wird. Das Integral 7) wird deshalb für diese Koordinatenwerte, wie es das Problem erfordert, logarithmisch unendlich.

Die Abb. 9 veranschaulicht den

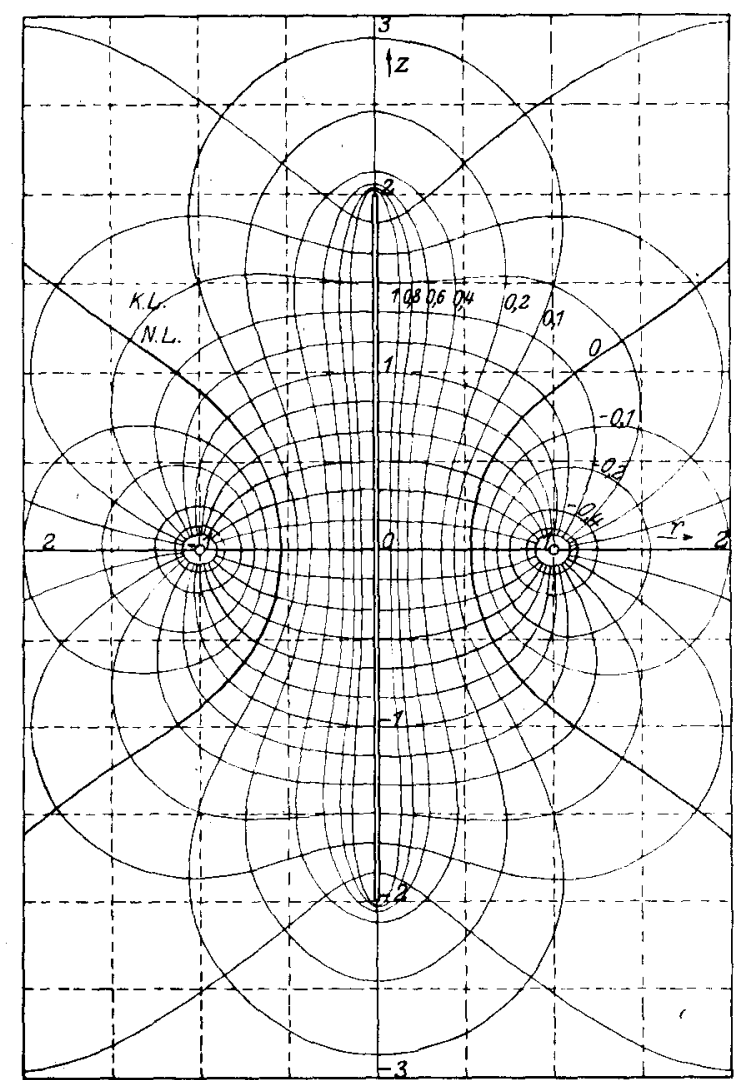

Abb. 9 . Kraft- und Niveaulinienverlauf für das resultierende Potential $\phi=\varphi_{1}+\varphi_{3}$ bei zusammenfallender Lage der Koordinatensysteme des Stab- und Ringpotentials $\left.{ }^{1}\right)$.

\section{B. Freileitungspotential.}

Um das elektrische Potential von Freileitungen näherungsweise bestim. men zu können, denken wir uns dieselben linear und die Erdoberfläche als leitende Ebene (E). In diesem Abschnitte stellen wir uns die Aufgabe, dasjenige Newtonsche Potential zu ermitteln, welches an den linearen Leitern willkïliche Randwerte im Sinne des Abschnittes über die linearen Leiter [ver. gleiche S. 22 bis 24] annimmt und an der Ebene $\mathrm{E}$ verschwindet. Nach dem dort Behandelten ergibt sich so die Möglichkeit, willkürliche Spannungsabfälle an endlich dimensionierten Freileitungen mit guter Annäherung $z u$ berücksichtigen.

Um die Aufgabe zu lösen, fassen wir die linearen Massenverteilungen als Superpositionen von Massenpolen auf. Irgendein solcher Massenpol $\mathrm{m}$

über der Ebene $\mathrm{E}$ hat ein Potential

$$
\varphi_{1}=\frac{m}{r}
$$

$[\mathrm{r}=$ Abstand des Aufpunktes vom Massenpol],

1) Abgelesen wurden die Werte des elliptischen Vollintegrals 7) aus der Funktionentafel von Jahnke und Emde liber das elliptische Vollintegral, erster Gattung, vergleiche dort S. $54-59$. 
welches an $\mathrm{E}$ durch das Potential

$$
\left.\varphi_{2}=-\frac{\mathrm{m}}{\mathrm{r}^{*}} \cdot \ldots \cdot . \cdot \ldots \cdot . \cdot 2\right)
$$

einer spiegelbildlich in bezug auf $\mathrm{E} z \mathrm{u} \mathrm{m}$ gelegenen Masse (- $\mathrm{m}$ ) zu Null kompensiert wird.

Das Freileitungspotential eines beliebigen Leiters L mit der Massenverteilung $\lambda$ [ $\lambda$ ist eine willkürliche, stetige Funktion der krummlinigen Koordinate $s$ des Leiters $L$ ] ist daher für den Aufpunkt $\mathrm{P}$ gegeben durch:

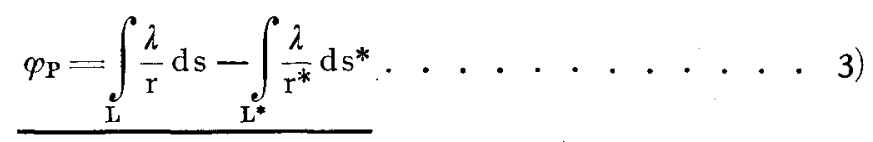

$\mathrm{r}=$ Abstand von $\mathrm{P}$ und $\mathrm{Q}$ [Punkt mit der Liniendichte $\lambda]$.

$\mathrm{r}^{*}=\quad, \quad, \mathrm{P}, \quad$ dem Spiegeibilde $\mathrm{Q}^{*}$ von $\mathrm{Q}$ in bezug auf $\mathrm{E}$.

Führt man dreifach rechtwinklige Koordinaten $(x, y, z)$ ein und läßt einfachheitshalber die $(\mathrm{x}, \mathrm{y})$-Ebene mit $\mathrm{E}$ zusammenfallen, so ist, wenn

und

$$
P \text { die Koordinaten } x, y, z
$$

besitzt:

$$
\mathrm{Q}, \quad, \quad \xi, \eta, \zeta
$$

$$
\left.\begin{array}{rl}
\mathrm{r} & =\sqrt{(\mathrm{x}-\xi)^{2}+(\mathrm{y}-\eta)^{2}+(\mathrm{z}-\zeta)^{2}} \\
\mathrm{r}^{*} & =\sqrt{(\mathrm{x}-\xi)^{2}+(\mathrm{y}-\eta)^{2}+(\mathrm{z}+\zeta)^{2}}
\end{array}\right\} \quad . . . . . .4 \text { 4) }
$$

Die Integrationen in 3) beziehen sich dann auf die Elemente ds und $\mathrm{ds}^{*}$ des linearen Leiters $L$ und dessen Spiegelbild $L^{*}$ zu $E$ mit den Liniendichten $\lambda_{\xi, \eta, \xi}$. In der Nähe irgendeines Quellpunktes $Q_{\xi, \eta, \xi}$ ist $\varphi_{\mathrm{P}}$ von der Form:

$$
\varphi_{\mathrm{Q}}=2 \lambda_{\mathrm{Q}} \cdot \lg \frac{\mathrm{I}}{\varrho}+\text { functio continua, . . . . . . . 5) }
$$

wobei f. c. gegenüber $2 \lambda_{Q} \lg \frac{\mathbf{I}}{\varrho}$ zu vernachlässigen ist. Die Liniendichte $\lambda$ für ein durch die willkürliche Randfunktion $I I$ gegebenes Freileitungspotential bestimmt sich auch hier, wie auf S. 23 durch:

$$
\left.\lambda=\frac{\Pi}{2 \lg \frac{\mathbf{I}}{\varrho}} \quad \ldots . . .6 . . .6 .6\right)
$$

$[2 \varrho=$ Dicke des endlichen Leiters].

Im praktisch wichtigsten Falle ist $\lambda$ in linearer Abhängigkeit von der krummlinigen Koordinate s; denn nach dem Ohmschen Gesetze gilt für die Spannungsverteilung längs eines dünnen Leiters:

$$
\Pi=\mathrm{a}+\mathrm{bs} \text {. . . . . . . . . . . . . 7) }
$$

Die Konstante a bestimmt den Nullpunkt des Potentials und die Konstante b das Spannungsgefälle bei überall gleichem Leiterquerschnitt durch:

$$
\mathrm{b}=\frac{\partial \Pi}{\partial \mathrm{s}}=\frac{\mathrm{j}}{\lambda_{\mathrm{L}}} \ldots . . . . . . .8
$$

$\left[\mathrm{j}=\right.$ Stromdichte $; \lambda_{\mathbf{L}}=$ Leitfähigkeit des Leitermaterials $]$.

Kommen mehrere Leitungen im Felde vor, so erhält man die resultierende Potentialfunktion durch Summation der Potentiale der einzelnen Leiter nach dem Superpositionsprinzipe. Auch Verzweigungen von Leitungen zu berücksichtigen, bietet nach den Kirchhoffschen Sätzen keine Schwierigkeiten. Für die neuen Leiterzweige 
kommen im allgemeinen auch neue Konstanten $\mathrm{a}$ und $\mathrm{b}$ in Betracht; diese hängen vom Potentialnullpunkt, der diesbezüglichen Stromdichte und Materialleitfähigkeit ab.

Die hier gegebene Näherungsmethode eignet, sich, wie leicht ersichtlich ist, zur Potentialbestimmung im umgebenden homogenen Dielektrikum von Gleichströmen und kann auch im Falle von nur nicht zu rasch oszillierenden Wechselströmen angewendet werden. Die Massen- und Potentialverteilung ist dann proportional der Stärke des Momentanstromes, bleibt sich jedoch immer ähnlich.

Anwendung auf praktisch wichtige Fälle.

A. Freileitung von endlicher Länge mit Berücksichtigung des Spannungsabfalles. Die meisten Freileitungen setzen sich aus einem oder mehreren horizontal geführten

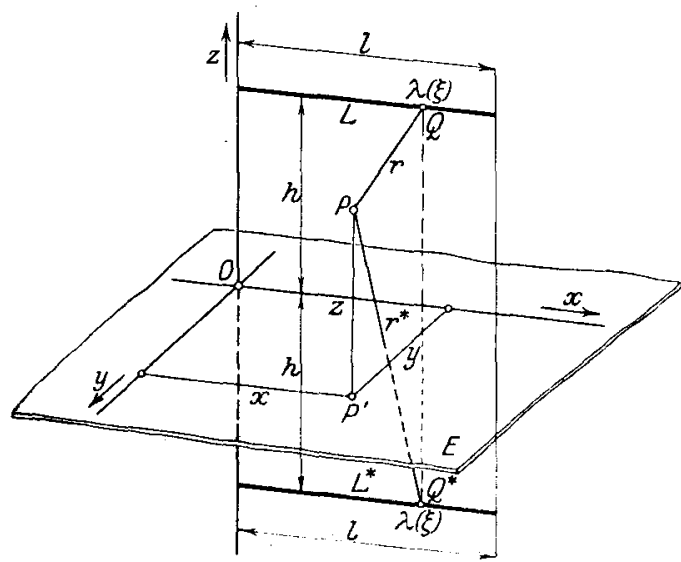

Abb. 10 Leiterstücken zusammen. Wenn man dieselben durch lin ea re Leiter ersetzt, gilt das Superpositionsprinzip; das resultierende Potential ist dann die Superposition der Potentiale der einzelnen Teilleiter. Rechnerisches Interesse bietet daher auch nur die Potentialbestimmung eines solchen Teilleiters. Ist $l$ die Länge desselben und $h$ dessen Abstand von der Erde, so erhält man nach 3) mit Hilfe von Abb. Io, wenn

$$
\lambda=\mathrm{a}+\mathrm{b} \xi
$$

ist, für das diesbezügliche Leiterpotential:

$\varphi_{\mathrm{x}, \mathrm{y}, \mathrm{z}}=\int_{0}^{1} \frac{\mathrm{a}+\mathrm{b} \xi}{\mathrm{r}_{\xi}} \mathrm{d} \xi-\int_{0}^{1} \frac{\mathrm{a}+\mathrm{b} \xi}{\mathrm{r}_{\xi}^{*}} \mathrm{~d} \xi$. 9)

wobei nun:

$$
\begin{aligned}
& r_{\xi}=\sqrt{(x-\xi)^{2}}+y^{2}+(z-h)^{2} \\
& r_{\xi}^{*}=\sqrt{(x-\xi)^{2}+y^{2}+(z+h)^{2}} .
\end{aligned}
$$

Die Ausführung der Integration liefert:

$$
\left.\varphi=(\mathrm{a}+\mathrm{bx}) \cdot \lg \left[\frac{\mathrm{r}_{1}+\mathrm{l}-\mathrm{x}}{\mathrm{r}_{1}^{*}+1-\mathrm{x}} \cdot \frac{\mathrm{r}_{0}^{*}-\mathrm{x}}{\mathrm{r}_{0}-\mathrm{x}}\right]+\mathrm{b}\left[\left(\mathrm{r}_{1}-\mathrm{r}_{1}^{*}\right)-\left(\mathrm{r}_{0}-\mathrm{r}_{0}^{*}\right)\right] . \quad . \quad \mathrm{I} 0\right)
$$

Der logarithmische Teil dieses Resultates kann nach Erweiterung des Numerus mit $\mathbf{r}_{0}+x$ geschrieben werden:

Hierin bedeutet:

$$
\left.(a+b x) \cdot \lg \left\{\frac{\left(r_{1}+1-x\right) \cdot\left(r_{0}+x\right) \cdot\left(r_{0}^{*}-x\right)}{\left(r_{1}^{*}+1-x\right) \cdot\left[y^{2}+(z-h)^{2}\right]}\right\} \ldots . . . \text { II }\right)
$$

$$
\left.\mathrm{y}^{2}+(\mathrm{z}-\mathrm{h})^{2}=\varrho^{2} \text {. . . . . . . . . . } \mathrm{r} 2\right)
$$

das Quadrat des Normalabstandes des Aufpunktes von der Leiterachse. Nun ist

$$
\begin{array}{c|c}
\text { für } y=0 & \text { und } \quad z=h: \\
r_{0}=x & r_{1}= \begin{cases}x-1 & \text { für } \quad \begin{array}{l}
x<0 \\
1-x
\end{array}, \quad 0<x<1\end{cases} \\
r_{0}{ }^{*}>x_{1} & r_{1}^{*} \mid>x-1 .
\end{array}
$$

Also ist der Ausdruck II) für $0<\mathrm{x}<\mathrm{l}$ von der Form:

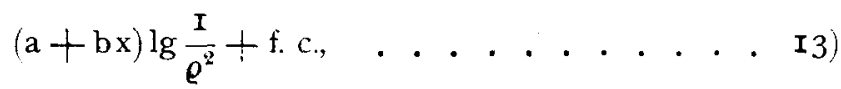


während für $\mathrm{x}<0$ und $\mathrm{x}>1$ dafür die unbestimmte Form $\frac{0}{0}$ auftritt. Damit ist im speziellen nachgewiesen, daß das Potential Formel ro) unter Zugrundelegung der Liniendichte $\lambda=a+b \xi$ am linearen Leiter Randwerte besitzt, wie sie Formel 5) entsprechen. Außerdem ist $\mathrm{zu}$ bemerken, daß für $\mathrm{z}=0$

$$
\mathrm{r}_{1}=\mathrm{r}_{1}^{*} \text { und } \mathrm{r}_{\mathrm{o}}=\mathrm{r}_{\mathrm{o}}{ }^{*}
$$

wird, so daß dort tatsächlich $\varphi$ verschwindet.

B. Spitzenpotential. Bei der Untersuchung von Potentialfeldern, die in der Nähe von Blitzableitern beim Ausströmen von ,statischer" Erdelek. trizität entstehen, kommt in erster Approximation das im folgenden berechnete Potential in Frage.

In Abb. II stehe der geradlinige, lineare Leiter s senkrecht zur Erdoberfläche. Die z-Achse des Zylinderkoordinatensystems $(r, z)$ falle damit zusammen, während die Ebene $z=0$ die Erde tangiert. $h_{1}$ sei die z-Koordinate des tiefsten und $h_{2}$ diejenige des höchsten Punktes des Leiters.

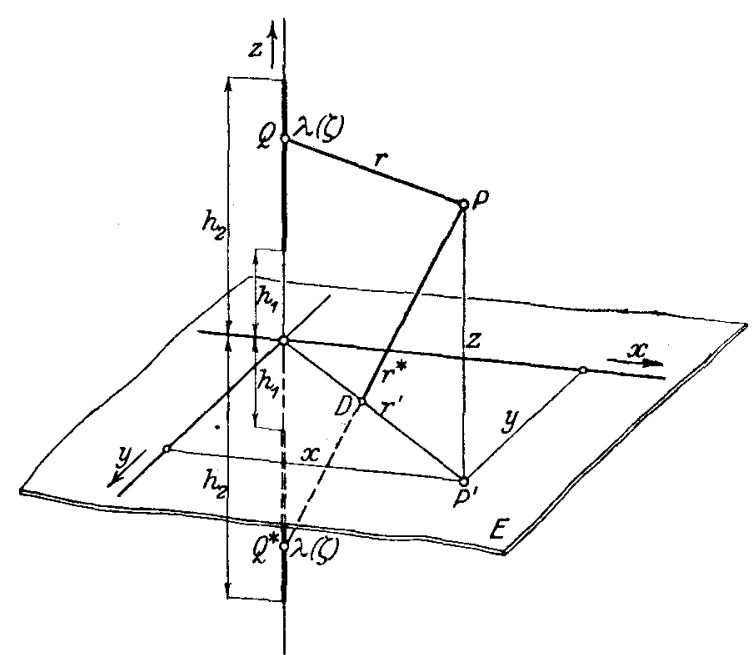

Abb. II.

Für das Potential des letzteren findet man nach Formel 9) bei linearer Verteilung der Spannung:

$$
\left.\varphi_{\mathrm{r}, \mathrm{z}}=\int_{\mathrm{h}_{1}}^{\mathrm{h}_{2}} \frac{\mathrm{a}+\mathrm{b} \zeta}{\sqrt{\mathrm{r}^{2}+(\mathrm{z}-\zeta)^{2}}} \mathrm{~d} \zeta-\int_{\mathrm{h}_{1}}^{\mathrm{h}_{2}} \frac{\mathrm{a}+\mathrm{b} \zeta}{\sqrt{\mathrm{r}^{2}+(\mathrm{z}+\zeta)^{2}}} \mathrm{~d} \zeta \quad \ldots . . \mathrm{I}_{4}\right)
$$

und, wenn man die folgenden Kürzungen einführt:

$$
\begin{aligned}
& \mathrm{r}^{2}+\mathrm{z}^{2}=\mathrm{R}^{2} \\
& \begin{array}{l|l}
\sqrt{\mathrm{R}^{2}-}-\overline{2 h_{1} z+h_{1}{ }^{2}}=A & \sqrt{R^{2}+2 h_{1} z+h_{1}{ }^{2}}=C \\
\sqrt{R^{2}-2 h_{2} z+h_{2}{ }^{2}}=B & \sqrt{R^{2}+2 h_{2} z+h_{2}{ }^{2}}=D
\end{array}
\end{aligned}
$$

nach Integration:

$\varphi=(\mathrm{a}+\mathrm{bz}) \lg \left[\frac{\mathrm{B}+\mathrm{h}_{2}-z}{\mathrm{~A}+\mathrm{h}_{1}-z}\right]-(\mathrm{a}-\mathrm{bz}) \cdot \lg \left[\frac{\mathrm{D}+\mathrm{h}_{2}+z}{\mathrm{C}+\mathrm{h}_{1}+\mathrm{z}}\right]+\mathrm{b}[(\mathrm{B}-\mathrm{A})-(\mathrm{D}-\mathrm{C})]$ I5)

$\mathrm{DaB}$ am Leiter:

$$
\lim _{\varrho=0}(\varphi)=(\mathrm{a}+\mathrm{bz}) \lg \frac{\mathrm{I}}{\varrho^{2}}+\mathrm{f} . \mathrm{c} .
$$

ist, läßt sich auf ähnliche Weise an I5) diskutieren, wie dies auf S. 30 mit der Potentialfunktion ro) geschah.

Die Vereinfachung $\mathrm{b}==0$ liefert die Formel:

$$
\left.\varphi=\mathrm{a} \cdot \lg \left[\frac{\mathrm{B}+\mathrm{h}_{2}-\mathrm{z}}{\mathrm{A}+\overline{\mathrm{h}_{1}}-\mathrm{z}+\mathrm{h}_{1}+\mathrm{z}}\right] \cdot \frac{\mathrm{D}+\mathrm{h}_{2}+\mathrm{z}}{\mathrm{D}}\right] .
$$

Mittelst linearer orthogonaler Koordinatentransformationen (Parallelverschiebung und Drehung des Koordinatensystems) ist es nach den Formeln ro) und I5) auf Grund des Superpositionsprinzips leicht möglich, das Potential ganzer Freileitungen anzugeben. 


\section{Isolatorproblem.}

Unter „Isolatorproblem“1) verstehe ich dasjenige elektrostatische Problem, welches sich auf die Kombination einer Kreislochscheibe und eines geradlinigen, linearen Leiters in der Achse derselben als Randwertkörper bezieht.

I. Der lineare Leiter ist von unbegrenzter Länge: Für diesen Fall führt der Weg, den Herr Prof. Dr. H. Weyl mir gewiesen hat, am schnellsten zum Ziele. Man kann nämlich den unendlich langen Leiter und die Kreislochscheibe auffassen als Grenzfälle von Rotationshyperboloiden einer konfokalen Schar. Eine solche Schar wird mittelst elliptischer Koordinaten durch die folgende Gleichung dargestellt:

$$
\frac{\mathrm{r}^{2}}{\mathrm{a}^{2}+\lambda^{*}}+\frac{\mathrm{z}^{2}}{\lambda^{*}}=\mathbf{I}
$$

wenn der Parameter $\lambda^{*}$ in den Grenzen $-\mathrm{a}^{2}<\lambda^{*}<0$ genommen wird. Im folgenden verwenden wir $\lambda=-\lambda^{*}$ als Parameter, wobei also: $0<\lambda<\mathrm{a}^{2}$. Die Potentialfunktion $\varphi$ ergibt sich dann aus dem berühmten Potentiale des Ellipsoides (vgl. darüber z. B. in "Kirchhoffs Vorlesungen“, Bd. IV, „Über das Potential des Ellipsoides") zu:

$$
\left.\varphi=\frac{\mathrm{m}}{2} \int_{0}^{\lambda} \frac{\mathrm{d} \lambda}{\left(\mathrm{a}^{2}-\lambda\right) \sqrt{\lambda}} \ldots . . . . .2\right)
$$

und nach Einführung der Substitution $\sqrt{\lambda}=\mu$ durch Integration:

und daraus folgt unmittelbar:

$$
\varphi=\frac{\mathrm{m}}{\mathrm{a}} \cdot \mathfrak{A} \mathfrak{r} \mathfrak{T} \mathfrak{g} \frac{\sqrt{\hat{\lambda}}}{\mathrm{a}}
$$

$$
\varphi=\frac{\mathrm{m}}{\mathrm{a}} \cdot \mathfrak{a r} \mathfrak{C} \mathfrak{v i j} \frac{\mathrm{a}}{\sqrt{\mathrm{a}^{2}-\lambda}} \text {. . . . . . . . . . . 4 }
$$

Aus 3) und 4) erkennt man, daß die Flächen $\varphi=$ konst. (Niveauflächen) sich ergeben durch die Bedingung $\lambda==$ konst. Auf Grund der Gleichung:

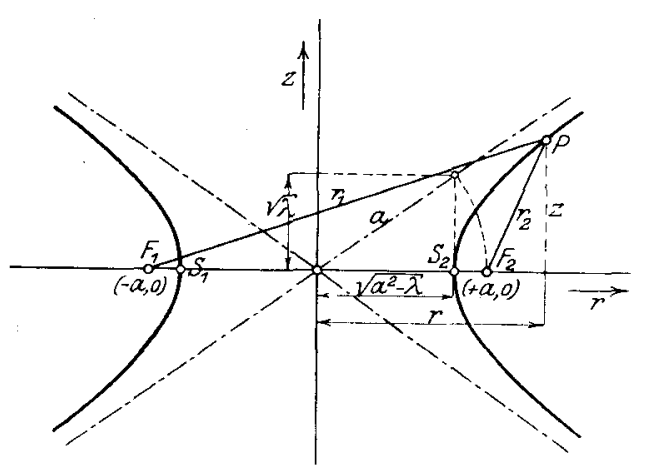

Abb. I 2.

$$
\left.\frac{\mathrm{r}^{2}}{\mathrm{a}^{2}-\lambda}-\frac{z^{2}}{\lambda}=\mathrm{I}, \ldots . \quad . \quad 5\right)
$$

die aus I) hervorgeht, wenn man dort $\lambda^{*}$ durch $-\lambda$ ersetzt, sind deshalb die Niveauflächen des berechneten Potentiales einschalige, konfokale Rotationshyperboloide. Die reelle Halbachse einer Meridianhyperbel hat die Länge $\sqrt{a^{2}-\bar{\lambda}}$, und die imaginäre Halbachse die Länge $\sqrt{\lambda}$. Da bei der Hyperbel die Differenz der beiden Brennstrahlen für jeden Punkt gleich der reellen Achse ist, gilt (vgl. dazu Abb. I2):

$$
\left.\sqrt{\mathrm{a}^{2}-\lambda}=\frac{\mathrm{r}_{1}-\mathrm{r}_{2}}{2}, \ldots . . . . . .7\right)
$$

wobei nach der Abbildung:

$$
\left.\left.\begin{array}{l}
r_{1}=\sqrt{(r+a)^{2}+z^{2}} \\
r_{2}=\sqrt{(r-a)^{2}+z^{2}}
\end{array}\right\} . . . . . . . . .8\right)
$$

1) Das hier vorliegende elektrische System ist die Idealisierung des bei den HochspannungsDurchführungsisolatoren vorkommenden Leitersystems. 
ist. Aus 4) geht mit 7) und 8) für das Potential $\varphi$ das Resultat hervor:

$$
\left.\varphi=\frac{\mathrm{m}}{\mathrm{a}} \mathfrak{A r} \mathfrak{e} \mathfrak{j} \frac{2 \mathrm{a}}{\sqrt{(\mathrm{r}+\mathrm{a})^{2}+\mathrm{z}^{2}}-\sqrt{(\mathrm{r}-\mathrm{a})^{2}+\mathrm{z}^{2}}} \cdot \ldots . . .99\right)
$$

Erweitert man das Argument des $\mathfrak{A r} \mathfrak{C} \mathfrak{D} \mathfrak{j}$ mit der Summe der beiden Wurzeln im Nenner, so folgt dafür auch:

$$
\varphi=\frac{m}{a} \mathfrak{A r} \operatorname{cof} \frac{\sqrt{(\mathrm{r}+\mathrm{a})^{2}+\mathrm{z}^{2}}+\sqrt{(\mathrm{r}-\mathrm{a})^{2}+z^{2}}}{2 \mathrm{r}}, \ldots . . . \text {. I0) }
$$

woraus hervorgeht, daB

$$
\lim _{\mathbf{r}=0}(\varphi)=\frac{\mathrm{m}}{\mathrm{a}} \cdot \lg \frac{\mathrm{I}}{\mathrm{r}}+\mathrm{f} . \mathrm{c} .
$$

ist und also die Niveaufächen des Potentials sich zylindrisch an einen linearen Kern in der z-Achse des Koordinatensystems anschließen, wie es bei einem statisch geladenen linearen Leiter, der überall von derselben Ordnung unendlich dünn ist, sein muß. Der lineare Leiter entspricht in der Hyperbelschar der Hyperbel mit dem Werte $\lambda=\mathrm{a}^{2}$.

Der Grenzübergang von Io) $z \mathrm{u} \mathrm{z}=\mathrm{o}$ liefert:

$$
(\varphi)_{\mathbf{z}=0}=\frac{m}{\mathrm{a}} \cdot \mathfrak{A r} \mathfrak{e v i} \frac{(\mathrm{a}+\mathrm{r}) \pm(\mathrm{a}-\mathrm{r})}{2 \mathrm{r}}
$$

wobei das positive Zeichen $z u$ verwenden ist, wenn $r<a$, und das negative, wenn $\mathrm{r}>\mathrm{a}$ ist. Daraus folgt aber:

$$
\left.\begin{array}{rl}
(\varphi)_{\mathrm{z}=0} & =\frac{\mathrm{m}}{\mathrm{a}} \cdot \mathfrak{A r} \operatorname{evo} \mathfrak{a} \frac{\mathrm{a}}{\mathrm{r}}, \text { wenn } \mathrm{r}<\mathrm{a} \\
& =0 \quad 0 \quad, \quad, \quad \mathrm{r}>\mathrm{a}
\end{array}\right\} \ldots .
$$

d. h. $\varphi$ verschwindet an der Kreislochscheibe, welche somit auch Niveaufläche des Potentials ist. Die Kreislochscheibe ist ein zweiter Grenzfall der Rotationshyperboloide für den Wert $\lambda=0$ und ist in der Auffassung als degeneriertes Rotationshyperboloid doppelblättrig.

Die Elektrizitätsdichte an der Kreislochscheibe erhält man nach Formel I6), Abschnitt I, aus:

$$
\eta=-\frac{\mathrm{I}}{2 \pi} \cdot\left(\frac{\partial \varphi}{\partial z}\right)_{\mathrm{z}=0}
$$

und also aus Io) durch Differentiation nach $z$ und den Grenzübergang zu $z=0$. Es ergibt sich so:

$$
\left.\eta=-\frac{\mathrm{m}}{2 \pi \mathrm{a} \sqrt{\mathrm{r}^{2}-\mathrm{a}^{2}}} \cdot . . . \mathrm{I} 2\right)
$$

Die Dichte wird danach am Rande der Kreis* lochscheibe unendlich.

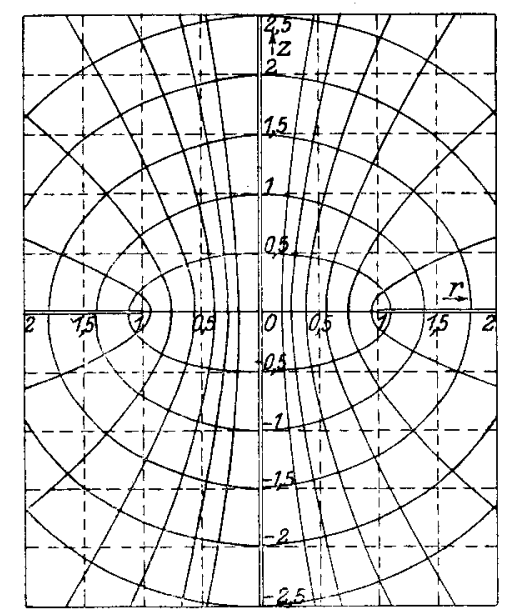

Abb. I3.

Durch Integration über die Liniendichte des unbegrenzten linearen Leiters einerseits und über die elektrische Dichte an der Scheibe andererseits folgert man leicht, daß die Gesamtmenge des Drahtes und der Scheibe einander entgegengesetzt gleich sind. Der Verlauf der Niveau- und Kraftlinien des Potentials 9 bzw. Io wird in Abb. I3 für einen Meridianschnitt dargestellt.

Willkürliche konzentrische Randwerte an der Kreislochscheibe: Sollen die Leiter des Isolatorproblems mit andern koaxialen Leitern kombiniert werden, so braucht man die Lösung des Isolatorproblems mit willkürlichen konzentrischen Rand. 
werten des Potentials an der Kreislochscheibe. Man kann zu diesem Zwecke zur Formel 9) dieses Paragraphen die in Abschnitt II Formel I4) gefundene allgemeine Kreislochscheibenlösung addieren. Die Potentialwerte der Kreislochscheibe werden dann durch jene Funktion dargestellt, während der Potentialwert des linearen Leiters durch den Faktor $\mathrm{m}$ von Gl. 9) dieses Abschnittes gegeben werden kann.

II. Der lineare Leiter ist von endlicher Länge: Es sollen für das Potential die Randwerte längs des linearen Leiters und eine konzentrische Verteilung an der Kreislochscheibe willkürlich vorgeschrieben sein. Wir haben dann eine Funktion zu bestimmen, die den Bedingungen genügt:

I. $\Delta \varphi=\frac{\partial^{2} \varphi}{\partial \mathrm{r}^{2}}+\frac{\mathrm{I}}{\mathrm{r}} \cdot \frac{\partial \varphi}{\partial \mathrm{r}}+\frac{\partial^{2} \varphi}{\partial \mathrm{z}^{2}}=0$

[ $\varphi$ verschwindet im Unendlichen].

II. $\varphi$ ist am linearen Leiter von der Form:

$$
\varphi=2 \lambda(\mathrm{z}) \lg \frac{\mathrm{I}}{\mathrm{r}}+\mathrm{f} . \mathrm{c} .
$$

sonst aber vollständig stetig.

III. $\varphi=\mathrm{F}(\mathrm{r})$ an der Kreislochscheibe

[also für $z=0, r>a$ ].

IV. Ableitungen von $\varphi$ stetig, ausgenommen am Stäbchen und in der Normalenrichtung an die Kreislochscheibe.

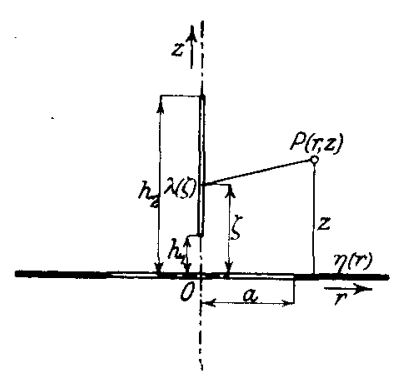

Abb. 14.

Bei der Lösung der Aufgabe führt uns das Superpositionsprinzip zum Ziele. Wir berechnen zuerst das Potential des linearen Stäbchens für sich und lagern darüber noch das entsprechende Kreislochscheibenpotential.

Das lineare Stäbchen werde begrenzt durch die z-Ordinaten $h_{1}$ und $h_{2}$ (vgl. dazu Abb. I4). Der Ausdruck des Stäbchenpotentials lautet dann nach unseren Unter. suchungen von S. 22 und 23 :

$$
\left.\varphi_{1}=\int_{\mathrm{h}_{1}}^{\mathrm{h}_{2}} \frac{\lambda(\zeta)}{\sqrt{\mathrm{r}^{2}+(\mathrm{z}-\zeta)^{2}}} \mathrm{~d} \zeta \ldots . . \quad \mathrm{r} 3\right)
$$

Um ein Potential mit der Randfunktion $F(r)$ an der Kreislochscheibe [Radius des Begrenzungskreises $=\mathrm{a}]$ zu erhalten, addieren wir zu $\varphi_{1}$ eine Kreislochscheibenlösung $\varphi_{2}$ mit der Randfunktion $F(r)-\left(\varphi_{1}\right)_{\mathrm{z}=0}$ an der Scheibe. Eine solche Funktion ist nach Abschnitt II möglich; ihre Flächendichte befindet sich ganz innerhalb der Scheibe und außerhalb derselben verläuft sie mit ihren Ableitungen stetig. Nach Abschnitt II, Formel I2) und Fußnote I von Seite 9 ist $\varphi_{2}$ der Realteil von

$$
\left.\varphi_{2}=\Re \int_{a}^{\infty} \frac{\mathrm{f}(\xi)}{\sqrt{(\xi+\mathrm{iz})^{2}-\mathrm{r}^{2}}} \mathrm{~d} \xi, \ldots . . . . . . \quad \mathrm{I}_{4}\right)
$$

und da $\varphi_{1}$ an der Kreislochscheibe regulär bleibt, ist die Flächendichte dort gegeben durch die Formel II), Abschnitt II, welche lautet

$$
\eta(\mathrm{r})=\frac{\mathrm{I}}{2 \pi \mathrm{r}} \cdot \frac{\mathrm{d}}{\mathrm{dr}} \int_{\mathrm{a}}^{\mathrm{r}} \frac{\mathrm{f}(\xi)}{\sqrt{\mathrm{r}^{2}-\xi^{2}}} \xi \mathrm{d} \xi, \quad . \quad . \quad . \quad . \quad . \quad \text {. I5) }
$$


wobei nach dem Besprochenen und Gl. Io), Abschnitt II zu setzen ist:

$$
\begin{aligned}
& \left.\mathrm{f}(\xi)=-\frac{2}{\pi} \cdot \frac{\mathrm{d}}{\mathrm{d} \xi} \int_{\xi}^{\infty} \frac{\Phi(\mathrm{r})}{\sqrt{\mathrm{r}^{2}-\xi^{2}}} \mathrm{r} \mathrm{d} \mathbf{r} \ldots . . . \quad . \quad . \quad \mathbf{I} 6\right) \\
& \left.\Phi(\mathrm{r})=\mathrm{F}(\mathrm{r})-\left|\varphi_{1}(\mathrm{r}, \mathrm{z})\right|_{\mathrm{z}=0} \quad . \quad . \quad . \quad . \quad . \quad . \quad \mathrm{I} 7\right) \\
& \left.\left|\varphi_{1}(\mathrm{r}, \mathrm{z})\right|_{\mathrm{z}=0}=\int_{\mathrm{h}_{1}}^{\mathrm{h}_{\mathrm{z}}} \frac{\lambda(\zeta)}{\sqrt{\mathrm{r}^{2}+\zeta^{2}}} \mathrm{~d} \zeta \ldots . . . . . . \mathrm{I}^{\mathrm{I}} 8\right)
\end{aligned}
$$

Führt man den Ausdruck I8) von $\left(\varphi_{1}\right)_{z}=0$ in I 7 ) ein, so tritt in I6) folgendes Doppelintegral auf:

worin:

$$
\frac{\mathrm{d}}{\mathrm{d} \xi} \int_{\xi}^{\infty} \int_{\mathrm{h}_{2}}^{\mathrm{h}_{2}} \lambda(\zeta) \frac{\mathrm{rdrd} \zeta}{\sqrt{\mathrm{r}^{2}}-\frac{\xi^{2}}{\sqrt{\mathrm{r}^{2}+\zeta^{2}}}}
$$

ist.

$$
\left.\lim _{\mathrm{g}=\infty}\left(\frac{\mathrm{d}}{\mathrm{d} \xi} \int_{\xi}^{\mathrm{g}} \frac{\mathrm{rdr}}{\sqrt{\mathrm{r}^{2}-\xi^{2}} \sqrt{\mathrm{r}^{2}+\zeta^{2}}}\right)=-\frac{\xi}{\xi^{2}+\zeta^{2}}{ }^{1}\right)
$$

Für unser Isolatorproblem wird daher bei den willkürlichen Randfunktionen des Potentials

$\mathrm{f}(\xi)$ allgemein dargestellt durch:

$$
\begin{aligned}
& \mathrm{F}(\mathrm{r}) \text { an der Kreislochscheibe und } \\
& \Pi(\mathrm{z})=2 \lambda(\mathrm{z}) \lg \frac{\mathrm{I}}{\varrho} \text { am Stableiter }
\end{aligned}
$$

$$
\begin{aligned}
& \left.\mathrm{f}(\xi)=-\frac{2}{\pi}\left[\frac{\mathrm{d}}{\mathrm{d} \xi} \int_{\xi}^{\infty} \frac{\mathrm{F}(\mathrm{r})}{\sqrt{\mathrm{x}^{2}-}-\xi^{2}} \mathrm{rdr}+\xi \int_{\mathrm{h}_{1}}^{\mathrm{h}_{2}} \frac{\lambda(\zeta)}{\xi^{2}+\zeta^{2}} \mathrm{~d} \zeta\right] . . . \text {. . I } 9\right) \\
& \left.\left.\begin{array}{rl}
\mathrm{h}_{2} & =-\mathrm{h}_{1}=\mathrm{h} \\
\lambda(\mathrm{z}) & =\frac{\mathrm{m}}{2 \mathrm{~h}}=\text { konst. } \\
\mathrm{F}(\mathrm{r}) & =\mathrm{o}
\end{array}\right\} \quad \cdot . \cdot . \cdot . \cdot 2 \mathrm{C}\right)
\end{aligned}
$$

Spezialisierung:

Um bei diesen vereinfachenden Annahmen $\eta(\mathbf{r})$ leicht berechnen zu können, formen wir das Integral in $\mathrm{r}_{5}$ ) partiell um. Es ist:

und folglich:

$$
\left.\int_{a}^{r} \frac{f(\xi)}{\sqrt{r^{2}-\xi^{2}}} \xi d \xi=f(a) \sqrt{r^{2}-a^{2}}+\int_{a}^{r} f^{\prime}(\xi) \cdot \sqrt{r^{2}-\xi^{2}} \cdot d \xi \ldots . . .2 I\right)
$$

$$
\left.\eta(\mathrm{r})=\frac{\mathrm{I}}{2 \pi}\left[\frac{\mathrm{f}(\mathrm{a})}{\sqrt{\mathrm{r}^{2}-\mathrm{a}^{2}}}+\int_{\mathrm{a}}^{\mathrm{r}} \frac{\mathrm{f}^{\prime}(\xi)}{\sqrt{\mathrm{r}^{2}-\xi^{2}}} \mathrm{~d} \xi\right] \ldots . . . .22\right)
$$

Ferner ergibt sich aus I9) und 20)

$$
\mathrm{f}(\xi)=-\frac{2}{\pi} \cdot \frac{\mathrm{m}}{\mathrm{h}} \int_{0}^{\mathrm{h}} \frac{\xi}{\xi^{2}+\zeta^{2}} \mathrm{~d} \zeta
$$

1) Nähern Aufschluf über die Notwendigkeit der hier angewandten Grenzwertbildung gibt G1. 2) der zur „Formeltabelle" gehörenden Fußnote. 
d. h. also nach Integration und nachträglicher Differentiation nach $\xi$ :

$$
\left.\left.\begin{array}{rl}
\mathrm{f}(\xi) & =-\frac{2}{\pi} \cdot \frac{\mathrm{m}}{\mathrm{h}} \operatorname{arctg}\left(\frac{\mathrm{h}}{\xi}\right) \\
\mathrm{f}^{\prime}(\xi) & =\frac{\mathrm{df}(\xi)}{\mathrm{d} \xi}=\frac{2}{\pi} \cdot \frac{\mathrm{m}}{\mathrm{h}^{2}+\xi^{2}}
\end{array}\right\} . . . . . .23\right)
$$

Substituiert man hieraus die Funktionen $f(a)$ und $f^{\prime}(\xi)$ nach 22) und wertet das dabei entstehende Integral

$$
\int_{\mathbf{a}}^{\mathbf{r}} \frac{\mathrm{d} \xi}{\left(\mathrm{h}^{2}+\xi^{2}\right) \sqrt{\mathrm{r}^{2}-\xi^{2}}}=-\left(\frac{\mathrm{I}}{\mathrm{h} \sqrt{\mathrm{r}^{2}+\mathrm{h}^{2}}} \operatorname{arccotg}\left(\frac{\xi \sqrt{\mathrm{r}^{2}+\mathrm{h}^{2}}}{\mathrm{~h} \sqrt{\mathrm{r}^{2}-\xi^{2}}}\right)_{\xi=\mathbf{a}}^{\xi=\mathbf{r}} \cdot \ldots .24\right)
$$

des zweiten Termes aus, so gelangt man zu dem folgenden Resultate für die konzentrische Verteilung der Dichte an der Kreislochscheibe:

$$
\eta(\mathrm{r})=-\frac{\mathrm{I}}{\pi^{2}} \cdot \frac{\mathrm{m}}{\mathrm{h}}\left[\frac{\operatorname{arctg}\left(\frac{\mathrm{h}}{\mathrm{a}}\right)}{\sqrt{\mathrm{r}^{2}-\mathrm{a}^{2}}}-\frac{\mathrm{I}}{\sqrt{\mathrm{r}^{2}+\mathrm{h}^{2}}} \operatorname{arccotg}\left(\frac{\mathrm{a}}{\mathrm{h}} \sqrt{\frac{\mathrm{r}^{2}+\mathrm{h}^{2}}{\mathrm{r}^{2}-\mathrm{a}^{2}}}\right)\right] .
$$

Damit ist aber das Problem der Elektrizitätsverteilung für den in 20) formulierten Spezialfall vollständig gelöst. Für $r=\infty$ folgt daraus, wenn gesetzt wird: $\lim _{r=\infty}(r)=R$

$$
\eta(\mathrm{R})=-\frac{\mathrm{I}}{\pi^{2}} \cdot \frac{\mathrm{m}}{\mathrm{h}}\left[\frac{\operatorname{arctg}\left(\frac{\mathrm{h}}{\mathrm{a}}\right)}{\mathrm{R}}-\frac{\operatorname{arccotg}\left(\frac{\mathrm{a}}{\mathrm{h}}\right)}{\mathrm{R}}\right],
$$

was in 2. Ordnung verschwindet. Es folgt daraus die Konvergenz des Integrals der zugehörigen Potentialfunktion.

Führt man in Gl. 25) die neue Konstante $\overline{\mathrm{m}}=\frac{\mathrm{a}}{\mathrm{h}} \mathrm{m}$ ein und macht dann die Spezialisierung $h=\infty$, so ergibt sich:

$$
\left.\overline{\eta(\mathrm{r})}=-\frac{\overline{\mathrm{m}}}{2 \pi \mathrm{a} \sqrt{\mathrm{r}^{2}-\mathrm{a}^{2}}} \cdot \ldots . . . .26\right)
$$

d. h. ein Resultat, das mit I2) vollständig im Einklange steht und nichts anderes bedeutet als die Flächendichte bei dem zuerst behandelten Isolatorproblem mit unendlich langem Leiter.

Für die Konstruktion des 22) entsprechenden Kraftlinienbildes benötigen wir noch den Ausdruck für die Elektrizitätsmenge zwischen beliebigen konzentrischen Kreisen an der Scheibe. Die Menge zwischen den Kreisen $[\mathrm{r}=\mathrm{a}]$ und $[\mathrm{r}=\mathrm{r}]$ ist:

und also nach 15$)$ gleich:

$$
\left.q(r)=\int_{0}^{2 \pi} \int_{a}^{r} \eta(r) r d r d y \quad . . . . . .27\right)
$$

$$
\left.q(r)=\int_{a}^{r} f(\xi) \frac{\xi d \xi}{\sqrt{r^{2}-\xi^{2}}} \cdot \ldots . . . . .28\right)
$$

und nach 2I):

$$
\mathfrak{q}(\mathrm{r})=\mathrm{f}(\mathrm{a}) \sqrt{\mathrm{r}^{2}-\mathrm{a}^{2}}+\int_{\mathrm{a}}^{\mathbf{r}} \mathrm{f}^{\prime}(\xi) \sqrt{\mathrm{r}^{2}-\xi^{2}} \cdot \mathrm{d} \xi \ldots . . \quad \text {. . 29) }
$$

Indem man in 29) die Funktionen $f(a)$ und $f^{\prime}(\xi)$ aus 23) einführt und dann das Integral wie folgt zerlegt: 


$$
\int_{a}^{r} \frac{\sqrt{r^{2}-\xi^{2}}}{h^{2}+\xi^{2}} d \xi=\left(h^{2}+r^{2}\right) \int_{a}^{r} \frac{d \xi}{\left(h^{2}+\xi^{2}\right) \sqrt{r^{2}-\xi^{2}}}-\int_{a}^{r} \frac{d \xi}{\sqrt{r^{2}-\xi^{2}}},
$$

ergibt sich mit Berücksichtigung von 24) und Ausfïhrung des zweiten Integrals für die elektrische Menge $\mathfrak{q}(\mathrm{r})$ folgender Ausdruck:

$\underline{q}(\mathrm{r})=-\frac{2}{\pi} \mathrm{m}\left[\frac{\sqrt{\mathrm{r}^{2}-\mathrm{a}^{2}}}{\mathrm{~h}} \cdot \operatorname{arctg}\left(\frac{\mathrm{h}}{\mathrm{a}}\right)-\frac{\sqrt{\mathrm{r}^{2}+\mathrm{h}^{2}}}{\mathrm{~h}} \cdot \operatorname{arccotg}\left(\frac{\mathrm{a}}{\mathrm{h}} \sqrt{\frac{\mathrm{r}^{2}+\mathrm{h}^{2}}{\mathrm{r}^{2}-\mathrm{a}^{2}}}\right)+\arccos \left(\frac{\mathrm{a}}{\mathrm{r}}\right)\right]$.

Die Menge $A q(r)$ zwischen zwei beliebigen Kreisen $r_{1}$ und $r_{2}\left[r_{1}>r_{2}\right]$ erhält man nach 28) aus:

$$
\mathfrak{q}\left(r_{1}\right)-q\left(r_{2}\right) \text {. }
$$

Setzt man $r=\infty$, so heben sich die beiden ersten Glieder der Klammer in 30) gegenseitig auf und das letzte Glied nimmt den Wert $\frac{\pi}{2}$ an. Die Gesamtmenge $q(R)$ der Scheibe ist demnach von der Größe:

$$
q(R)=-m \cdot \ldots \cdot 3 r)
$$

und dies ist, wie sich mit 20) ergibt:

$$
\mathfrak{q}(\mathrm{z})=\int_{-\mathrm{b}}^{+\mathrm{h}} \frac{\mathrm{m}}{2 \mathrm{~h}} \mathrm{~d} z=+\mathrm{m}
$$

genau die entgegengesetzt gleiche Menge des linearen Leiters.

Für das Potential $\varphi=\varphi_{1}+\varphi_{2}$ hat man nach I3), I4) und 23) den Ausdruck:

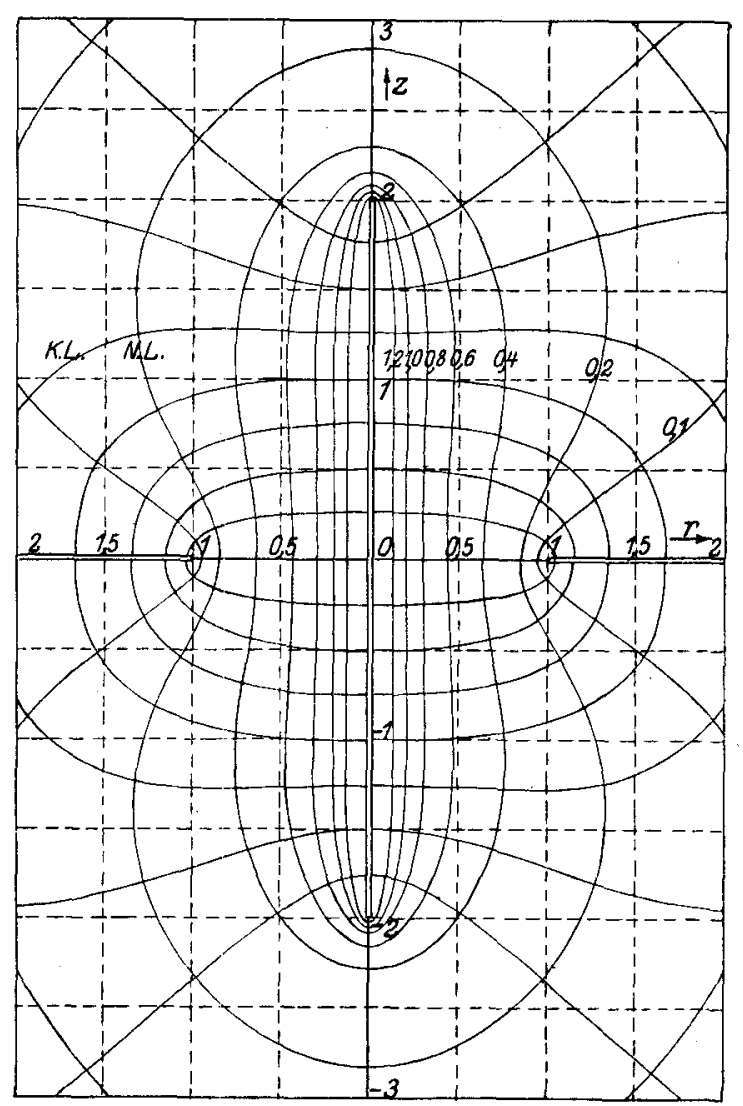

Abb. 15 .

$$
\varphi=\frac{\mathrm{m}}{2 \mathrm{~h}} \int_{-\mathrm{h}}^{+\mathrm{h}} \frac{\mathrm{d} \zeta}{\sqrt{\mathrm{r}^{2}}+(\mathrm{z}-\bar{l})^{2}}-\frac{2}{\pi} \cdot \frac{\mathrm{m}}{\mathrm{h}} \Re \int_{a}^{\infty} \frac{\operatorname{arctg} \frac{\mathrm{h}}{\xi}}{\sqrt{(\xi+\mathrm{i})^{2}-\mathrm{r}^{2}}} \mathrm{~d} \xi, \ldots .
$$

wobei man den ersten Term in Abschnitt VI vollständig integriert findet, während der zweite Term nur durch Reihenentwicklung oder näherungsweise durch mechanische Quadratur integrierbar ist. Das Kraftlinienbild von $\varphi$ [Abb. I5] wurde unter Zuhilfenahme von graphischer Integration erhalten.

\section{Axiale Verteilung an einem unendlich langen Rotationszylinder.}

Bei verschiedenen für die Anwendung wichtigen elektrostatischen Systemen, bei welchen zylindrische Leiter von kreisförmigem Querschnitte vorkommen, benötigt man die allgemeine Lösung eines Potentials, das durch eine willkürliche axiale Verteilung an einer Zylinderoberfläche gegeben ist. 
Nach einem bekannten Satze der Potentialtheorie ist das Potential innerhalb eines geschlossenen Raumes vollständig bestimmt durch die Potentialwerte an der Oberfläche dieses Raumes ${ }^{1}$ ). Dies ermöglicht unter der Voraussetzung, daß $\varphi$ im Unendlichen verschwindet, eine Zerlegung unserer Aufgabe. Wir berechnen die Potentiale $\varphi^{(\mathrm{a})}$ und $\varphi^{(\mathrm{i})}$ des Zylinderaußen- und -innenraumes je für sich.

Gemeinsam ist für die beiden Funktionen $\varphi^{(\mathrm{a})}$ und $\varphi^{(i)}$, daß sie überall stetig verlaufen und im Unendlichen verschwinden [die Randfunktion am Zylinder ist dafür entsprechend zu wählen], ferner, daß sie beide der Differentialgleichung:

$$
\text { I. } \Delta \varphi=\frac{\partial^{2} \varphi}{\partial \mathrm{r}^{2}}+\frac{\mathrm{I}}{\mathrm{r}} \cdot \frac{\partial \varphi}{\partial \mathrm{r}}+\frac{\partial^{2} \varphi}{\partial \mathrm{z}^{2}}=0
$$

genügen und längs der Zylinderfläche in dieselbe willkürliche Randfunktion

ubergehen.

$$
\text { II. } \quad \mathrm{F}(\mathrm{z})
$$

Zur Integration von Gl. I verwenden wir den folgenden Ansatz:

$$
\varphi_{\mathrm{p}}=\cos (\alpha z) \Phi(\mathrm{r})+\sin (\alpha z) \Psi(\mathrm{r})
$$

$[\Phi(\mathrm{r})$ und $\Psi(\mathrm{r})$ sollen nur Funktionen von $\mathrm{r}$ allein sein].

Für $\Phi$ und $\Psi$ gelten dann die Differentialgleichungen:

$$
\left.\begin{array}{l}
\frac{\mathrm{d}^{2} \Phi}{\mathrm{dr}^{2}}+\frac{\mathrm{I}}{\mathrm{r}} \cdot \frac{\mathrm{d} \Phi}{\mathrm{dr}}-\alpha^{2} \Phi=0 \\
\frac{\mathrm{d}^{2} \Psi}{\mathrm{d} \mathrm{r}^{2}}+\frac{\mathrm{I}}{\mathrm{r}} \cdot \frac{\mathrm{d} \Psi}{\mathrm{dr}}-\alpha^{2} \Psi=0
\end{array}\right\}
$$

Diese Gleichungen werden erfült durch die Besselschen Funktionen nullter Ordnung von rein imaginärem Argument. Für irgendeine solche Besselfunktion wollen wir einstweilen die Bezeichnung $\mathrm{B}(\mathrm{i} \alpha \mathbf{r})$ einführen. Gl. I wird dann erfüllt durch ein Partikularintegral von der Form:

$$
p_{\mathrm{P}}=\cos (\alpha \mathrm{z}) \mathrm{B}(\mathrm{i} \kappa \mathrm{r}) \mathrm{f}_{1}(\alpha)+\sin (\alpha \mathrm{z}) \mathrm{B}(\mathrm{i} \alpha \mathrm{r}) \mathrm{f}_{2}(\alpha) \text {. . . . . . 3) }
$$

und durch das allgemeine Integral:

$$
\left.\varphi==\int_{0}^{\infty} \mathrm{B}(\mathrm{i} \alpha \mathrm{r})\left[\cos (\alpha z) \mathrm{f}_{1}(\alpha)+\sin (\alpha \mathrm{z}) \mathrm{f}_{2}(\alpha)\right] \mathrm{d} \alpha \ldots . . .44\right)
$$

Um auch der Bedingung II zu genügen, verfügen wir über $f_{1}(\alpha)$ und $f_{2}(\alpha)$ so, daß nach dem Fourierschen Integraltheorem [Formeltabelle I, C] an der Zylinderoberfläche für das Potential $\varphi$ der Randwert $F(z)$ herauskommt. - Bezeichnet $R$ den Zylinderradius, so ist zu setzen:

Es folgt so:

$$
\left.\begin{array}{l}
\mathrm{f}_{1}(\alpha)=\frac{\mathrm{r}}{\pi \cdot \mathrm{B}(\mathrm{i} \alpha \mathrm{R})} \int_{-\infty}^{+\infty} \mathrm{F}(\lambda) \cos (\alpha \lambda) \mathrm{d} \lambda \\
\mathrm{f}_{2}(\alpha)=\frac{\mathrm{I}}{\pi \cdot \mathrm{B}(\mathrm{i} \alpha \mathrm{R})} \int_{-\infty}^{+\infty} \mathrm{F}(\lambda) \sin (\alpha \lambda) \mathrm{d} \lambda
\end{array}\right\}
$$

$$
\left.\varphi=\frac{\mathrm{I}}{\pi} \int_{0}^{\infty} \frac{\mathrm{B}(\mathrm{i} \alpha \mathrm{r})}{\mathrm{B}(\mathrm{i} \alpha \mathrm{R})} \cdot\left[\cos (\alpha \mathrm{z}) \int_{-\infty}^{+\infty} \mathrm{F}(\lambda) \cos (\alpha \lambda) \mathrm{d} \lambda+\sin (\alpha \mathrm{z}) \int_{-\infty}^{+\infty} \mathrm{F}(\lambda) \sin (\alpha \lambda) \mathrm{d} \lambda\right] \mathrm{d} \alpha \ldots \cdot 6\right)
$$

1) Vergleiche dazu die Fufnote von S. 22. 
und nach Vereinigung der innern Integrale:

$$
\left.\varphi=\frac{\mathrm{I}}{\pi} \int_{0}^{\infty} \frac{\mathrm{B}(\mathrm{i} \alpha \mathrm{r})}{\mathrm{B}(\mathrm{i} \alpha \mathrm{R})} \mathrm{d} \alpha \int_{-\infty}^{+\infty} \mathrm{F}(\lambda) \cos \alpha(\mathrm{z}-\lambda) \mathrm{d} \lambda \ldots \ldots 7\right)
$$

Weil die Differentialgleichungen 2) von der zweiten Ordnung sind, existieren nur zwei linear unabhängige Besselfunktionen nullter Ordnung $\mathrm{B}(\mathrm{ix})$; nämlich die Besselfunktion „erster Art" J(ix) und die Besselfunktion ,zweiter Art“ N(ix) [Neumannsche Zylinderfunktion]. Beide verlaufen für $0<\mathrm{x}<\infty$ durchaus monoton und besitzen an den Grenzen $\mathrm{x}=0$ und $\mathrm{x}=\infty$ die aus der folgenden Tabelle ersichtlichen speziellen Werte:

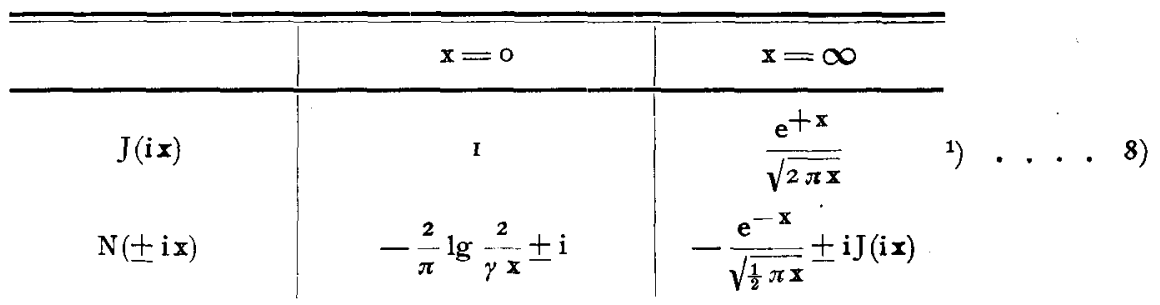

Bezeichnen wir in 7):

$$
\left.\left.\begin{array}{l}
\frac{\mathrm{B}(\mathrm{i} \alpha \mathrm{r})}{\mathrm{B}(\mathrm{i} \alpha \mathrm{R})}=\psi(\alpha) \\
\frac{\mathrm{I}}{\pi} \int_{-\infty}^{+\infty} \mathrm{F}(\lambda) \cos \alpha(\mathrm{z}-\lambda) \mathrm{d} \lambda=\varphi(\alpha)
\end{array}\right\}, \ldots . . . .99\right)
$$

so ist $\varphi(\alpha)$ und das Doppelintegral in 7) konvergent, wenn:

$$
\left.F(z)=\frac{I}{\pi} \int_{0}^{\infty} \mathrm{d} \alpha \int_{-\infty}^{+\infty} \mathrm{F}(\lambda) \cos \alpha(z-\lambda) \mathrm{d} \lambda \quad . \quad . . . . \text { I }\right)
$$

den Fourierschen Bedingungen gemäß gegeben ist [vergleiche darüber: RiemannWeber, Partielle Differentialgleichungen, Bd. I, S. 47] und $\psi(\alpha)$ von einem bestimmten $\alpha$ an mit wachsendem $\alpha$ beständig abnimmt und sich dabei für $\alpha=\infty$ stetig (asymptotisch) der Grenze Null nähert ${ }^{2}$ ), d. h. es muß also sein:

$$
\left.\lim _{\alpha=\infty}\left(\frac{B(i \alpha r)}{B(i \alpha R)}\right)=0 . . . . . . . . I I\right)
$$

Wegen des eigentümlichen Verhaltens der Funktionen $\mathrm{J}(\mathrm{ix})$ und $\mathrm{N}(\mathrm{ix})$ für ein unendlich großes Argument [nach den asymptotischen Darstellungen in 8) werden dafür nämlich beide unendlich wie $\left.\frac{\mathrm{e}^{+\mathrm{x}}}{\sqrt{\mathrm{x}}}\right]$, müssen wir, um der Bedingung II) Rechnung zu tragen, zwei Fälle unterscheiden; nämlich wo $r<R$ und wo $r>R$ ist.

Zylinderinnenraum $[\mathrm{r}<\mathrm{R}]$ : Die einzige Funktion $\mathrm{B}(\mathrm{ix})$, die hierfür in Betracht kommt, ist $\mathrm{J}(\mathrm{ix})$; diese bleibt nämlich an der Grenze $\mathrm{x}=0$ mit ihren Ableitungen stetig [was für $\mathrm{N}(\mathrm{ix})$ nicht zutrifft]; auch steht, solange wenigstens $r<R$ ist, $J(\mathrm{i} \alpha \mathbf{r})$ zu $J(i \alpha R)$ in einem solchen Verhältnis, daß: und 100

1) Siehe z. B. Jahnke und Emde, Funktionentafeln, Verlag B. G. Teubner 1909, S. 92,96

2) Vergleiche dartiber: Riemann.Weber, Partielle Differentialgleichungen, 5. Aufl., Bd. I, S. 17 oben. 


$$
\begin{aligned}
& \text { - } 40-
\end{aligned}
$$

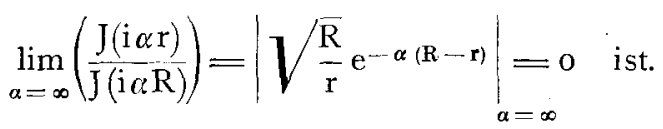

Für den Zylinderinnenraum haben wir deshalb die folgende allgemeine Lösung für das Potential:

$$
\varphi^{(i)}=\frac{\mathrm{I}}{\pi} \int_{0}^{\infty} \frac{\mathrm{J}(\mathrm{i} \alpha \mathrm{r})}{\mathrm{J}(\mathrm{i} \alpha \mathrm{R})} \mathrm{d} \alpha \int_{-\infty}^{+\infty} \mathrm{F}(\lambda) \cos \alpha(\mathrm{z}-\lambda) \mathrm{d} \lambda
$$

die für $r=R$ stetig in die Randfunktion $F(z)$ übergeht.

Zylinderaußenraum [ $r>R]$ : Für den Fall, daB $r>R$ ist, kann für $B(i x)$ keines der Partikularintegrale $\mathrm{J}(\mathrm{ix})$ und $\mathrm{N}(\mathrm{ix})$ der Differentialgleichungen 2) allein verwendet werden, weil beide für $\mathrm{x}=\infty$ wie $\frac{\mathrm{e}^{+\mathrm{x}}}{\sqrt{\mathrm{x}}}$ unendlich werden. Nach 8) wäre dann, abgesehen von nebensächlichen Konstanten

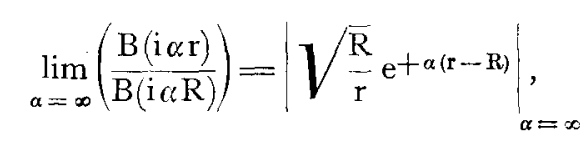

also sicher von sehr hoher Ordnung [im Grenzwert sogar unendlich hoher Ordnung] unendlich. Die einzige Funktion, die den Differentialgleichungen 2) genügt und II) nicht widerspricht, ist die folgende lineare Kombination:

$$
\left.\mathrm{H}( \pm \mathrm{ix})=\mathrm{N}\left(\mp_{\mathrm{ix}}\right) \pm \mathrm{i} J(\mathrm{ix}), \ldots . . . . . . . \mathrm{I} 3\right)
$$

die sog. „Hankelsche Zylinderfunktion“, aus den Partikularintegralen J(ix) und $\mathrm{N}(\mathrm{ix})$. Nach 8) gilt dafür die asymptotische Darstellung:

$$
\left.\mathrm{H}(\underset{\mathrm{x}=\infty}{ \pm \mathrm{ix}})=-\frac{\mathrm{e}^{-\mathrm{x}}}{\sqrt{\frac{1}{2} \pi \mathrm{x}}}, \ldots . . . . . \mathrm{I} 4\right)
$$

so daß:

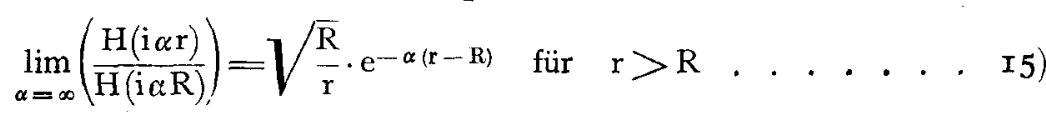

ist; ein Ausdruck, der also im Grenzwert stark gegen Null konvergiert. - Das Potential des Außenraumes wird daher allgemein dargestellt durch:

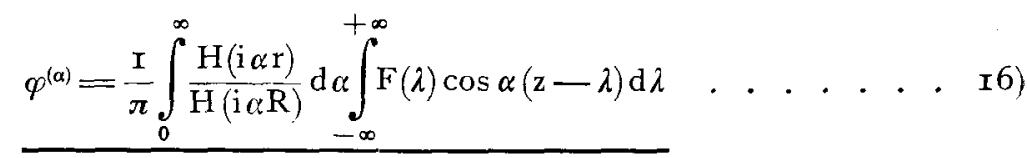

\section{Zylinder-Hohlraumpotential.}

In irgendeinem Punkte der Achse eines hohlen, leitenden und im Unendlichen

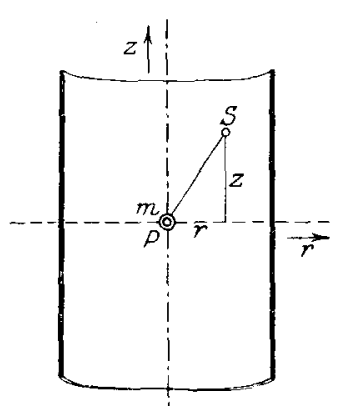

Abb. 16 . geerdeten Rotationszylinders befinde sich ein elektrischer Pol $\mathrm{P}$ mit der Masse $\mathrm{m}$ (s. Abb. I6). Der Pol P erregt im Zylinderhohlraume ein elektrisches Feld mit für die Zylinderinnenfläche verschwindenden Potentialwerten. Bei der Ermittlung der für den Zylinderhohlraum geltenden Potentialfunktion $\varphi$ verwenden wir das Superpositionsprinzip und bestimmen zum Potentiale

$$
\left.\varphi_{1}=\frac{\mathrm{m}}{\sqrt{\mathrm{r}^{2}+\mathrm{z}^{2}}} \cdot \ldots . . . . \mathrm{I}\right)
$$

des Poles ein Potential $\varphi_{2}$, das an der Zylinderfläche [Zy- 
linderradius $=\mathrm{R}$ ] die entgegengesetzt gleiche Randfunktion

$$
\left.\mathrm{F}(\mathrm{z})=-\frac{\mathrm{m}}{\sqrt{\mathrm{R}^{2}+\mathrm{z}^{2}}} \cdot \ldots . . . . . .2 \mathrm{z}\right)
$$

wie dieses besitzt und im Zylinderinnern vollständig stetig verläuft. Wir haben dazu bloß im Integrale, Formel I2) des vorigen Abschnittes, F(z) Gl. 2) entsprechend zu wählen. Für $\varphi_{2}$ gilt also:

$$
\left.\varphi_{2}=-\frac{\mathrm{m}}{\pi} \int_{0}^{\infty} \frac{\mathrm{J}(\mathrm{i} \alpha \mathrm{r})}{\mathrm{J}(\mathrm{i} \alpha \mathrm{R})} \mathrm{d} \alpha \int_{-\infty}^{+\infty} \frac{\cos \alpha(\mathrm{z}-\lambda)}{\sqrt{\overline{\mathrm{R}^{2}+\lambda^{2}}}} \mathrm{~d} \lambda, \ldots . . . .3\right)
$$

und da für jede ,ggerade Funktion“ F(z)

$$
\int_{-\infty}^{+\infty} \mathrm{F}(\lambda) \cos \alpha(z-\lambda) \mathrm{d} \lambda=2 \cos (\alpha z) \int_{0}^{\infty} \mathrm{F}(\lambda) \cos (\alpha \lambda) \mathrm{d} \lambda \ldots \ldots \text {. . . 4) }
$$

ist, muß auch sein:

Hierin ist aber:

$$
\left.\varphi_{2}=-\frac{2}{\pi} \mathrm{m} \int_{0}^{\infty} \cos (\alpha \mathrm{z}) \cdot \frac{\mathrm{J}(\mathrm{i} \alpha \mathrm{r})}{\mathrm{J}(\mathrm{i} \alpha \mathrm{R})} \mathrm{d} \alpha \int_{0}^{\infty} \frac{\cos (\alpha \lambda)}{\sqrt{\mathrm{R}^{2}+\lambda^{2}}} \mathrm{~d} \lambda . . . . .5\right)
$$

$$
\left.\mathrm{H}(\mathrm{i} \alpha \mathrm{R})=\frac{2}{\pi} \int_{0}^{\infty} \frac{\cos (\alpha \lambda)}{\left.\left.\sqrt{\mathrm{R}^{2}+\lambda^{2}} \mathrm{~d} \lambda\right)^{1}\right) . . . . . .} .6\right)
$$

nichts anderes als eine exakte Integraldarstellung für die Hankelsche Zylinderfunktion $\mathrm{H}(+\mathrm{i} \kappa \mathrm{R})$.

1) Für das hier vorkommende Integral kann man nämlich auch schreiben:

$$
\frac{1}{\pi} \int_{-\infty}^{+\infty} \frac{\cos (\alpha \lambda)+i \sin (\alpha \lambda)}{\sqrt{R^{2}+\bar{\lambda}^{2}}} d \lambda \ldots \ldots \ldots
$$

Der Integrationsweg erstreckt sich für ein reelles $\lambda$ in der , $\lambda$-Ebene" über die reelle Achse von $\lambda=-\infty$ bis $\lambda=+\infty$ und ist für ein imaginäres $\lambda=i \mu$ nach dem Integralsat $z$ von Cauchy zu ersetzen durch den "Schleifenweg" $\left(u_{1}, s_{1}, s_{9}, u_{2}\right)$. (Vergleiche nebenstehende Figur.) Da nun die Integralbeiträge von den Intervallen $u_{1}$ und $u_{2}$ gleich o

$$
\left[\text { folgt aus } \lim _{,=\infty}\left(\frac{\mathbf{I}}{\sqrt{\mathbf{R}^{2}+\lambda^{2}}}\right)=0\right]
$$

und diejenigen der Intervalle $s_{1}$ und $s_{2}$ anf der imaginären Achse absolut gleich sind, geht fuir $\lambda=\mathbf{i} \mu$ aus (I) hervor:

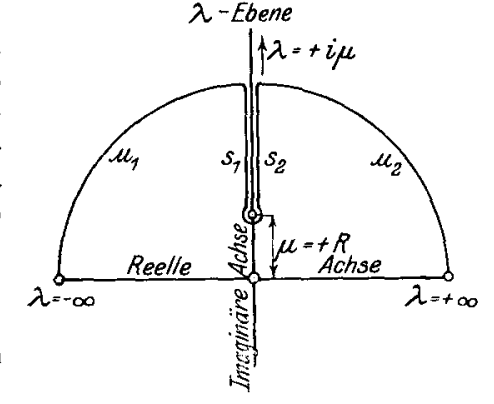

$$
\frac{2}{\pi} \int_{\mathrm{R}}^{\infty} \frac{\mathrm{e}^{-a \mu}}{\sqrt{\mu^{2}-\mathrm{R}^{2}}} \mathrm{~d} \mu \ldots \ldots . . . \ldots \text {. }(\mathrm{I})
$$

Nach der dritten Gleichung auf Seite 489 von Riemann-Weber, Partielle Diff.-Gl. Bd. I, 5. Aufl. und G1. 8) und I3) des vorhergehenden Abschnittes ist II aber identisch mit der Hankelschen $Z$ ylinderfunktion $\mathrm{H}(\mathbf{i} \alpha \mathbf{R})$ und besitzt, was für die nachfolgenden Betrachtungen wesentlich ist, für $\alpha=\infty$ die asymptotische Darstellung:

$$
-\frac{\mathrm{e}^{-\alpha \mathrm{R}}}{\sqrt{\frac{1}{2} \pi \alpha \mathrm{R}}}
$$


Für das Potential $\varphi_{2}$ hat man daher folgendes Integral:

$$
\left.\varphi_{\mathrm{a}}=-\mathrm{m} \int_{0}^{\infty} \cos (\alpha \mathrm{z}) \frac{\mathrm{J}(\mathrm{i} \alpha \mathrm{r})}{\mathrm{J}(\mathrm{i} \alpha} \frac{\mathrm{R})}{\mathrm{R}} \cdot \mathrm{H}(\mathrm{i} \alpha \mathrm{R}) \mathrm{d} \alpha \ldots . .7\right)
$$

Um dieses Integral durch elementare Funktionen ausdrücken zu können, entwickeln wir den Quotienten $\frac{\mathrm{J}(\mathrm{i} \kappa \mathrm{r})}{\mathrm{J}(\mathrm{i} \alpha \mathrm{R})}$ mit Hilfe der für jedes reelle und komplexe $\mathrm{x}$ gültigen Reihe:

$$
\left.J(i x)=I+\frac{\left(\frac{I}{2} x\right)^{2}}{I !^{2}}+\frac{\left(\frac{I}{2} x\right)^{4}}{2 !^{2}}+\frac{\left(\frac{I}{2} x\right)^{6}}{3 !^{2}}+\ldots . . . . .8\right)
$$

in eine Potenzreihe durch den Ansatz:

$$
\left.J(i \alpha r)=J(i \alpha R) \cdot\left[a_{0}+a_{1} \alpha+a_{2} \alpha^{2}+a_{3} \alpha^{3}+\ldots\right] . . . . .99\right)
$$

$\mathrm{Da}$ in den Reihen von J(i $\alpha \mathrm{r})$ und $\mathrm{J}(\mathrm{i} \alpha \mathrm{R})$ nur Potenzen von $\alpha$ mit geraden Exponenten vorkommen, ergibt sich leicht, daß in der Reihenentwicklung alle Koeffizienten mit ungeradem Index Null sind. Das Integral in 7) ist deshalb von der Form:

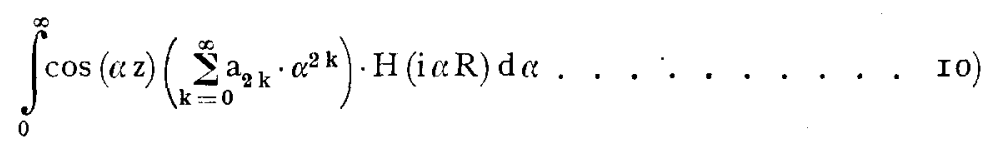

und darf hier gliedweise ausgeführt werden. Lm mich kurz fassen zu können, will ich mich auf den diesbezüglichen Beweis nicht weiter einlassen; doch sei bemerkt, daß er sich der gleichmäßigen Konvergenz der Reihe und der für $\alpha=\infty$ monoton zu $\frac{\mathrm{e}^{-\alpha \mathrm{R}}}{\sqrt{a \mathrm{R}}}$ verlaufenden Funktion $\mathrm{H}(\mathrm{i} \alpha \mathrm{R})$ wegen relativ einfach erbringen läßt.

Für Io) ist dann also zu schreiben:

$$
\sum_{\mathrm{k}=0}^{\infty} \mathrm{a}_{2 \mathrm{k}} \int_{0}^{\infty} \cos (\alpha \mathrm{z}) \cdot \alpha^{2 \mathrm{k}} \cdot \mathrm{H}(\mathrm{i} \alpha \mathrm{R}) \mathrm{d} \alpha=\sum_{\mathrm{k}=0}^{\infty}(-\mathrm{I})^{\mathrm{k}} \cdot \mathrm{a}_{2 \mathrm{k}} \cdot \frac{\mathrm{d}^{2 \mathrm{k}}}{\mathrm{d}^{2 \mathrm{k}}}\left(\int_{0}^{\infty} \cos (\alpha \mathrm{z}) \mathrm{H}(\mathrm{i} \alpha \mathrm{R}) \mathrm{d} \alpha\right) .
$$

Um das Integral auswerten zu können, verwenden wir die Integraldarstellung 6) von $\mathrm{H}(\mathrm{i} a \mathrm{R})$ und berücksichtigen das Fouri ersche Integraltheorem (Formeltabelle I, B). Es folgt so:

$$
\int_{0}^{\infty} \cos (\alpha z) \mathrm{H}(\mathrm{i} \alpha \mathrm{R}) \mathrm{d} \alpha=\frac{2}{\pi} \int_{0}^{\infty} \cos (\alpha \mathrm{z}) \mathrm{d} \alpha \int_{0}^{\infty} \frac{\cos (\alpha \lambda)}{\sqrt{\mathrm{R}^{2}+\lambda^{2}}} \mathrm{~d} \lambda=\frac{\mathrm{I}}{\sqrt{\mathrm{R}^{2}+\mathrm{z}^{2}}}
$$

und also mit Berücksichtigung der Konstanten (-m) in 7) für das Potential $\varphi_{2}$ die Reihe:

$$
\left.\varphi_{2}=-\sum_{\mathrm{k}=0}^{\infty}(-\mathrm{I})^{\mathrm{k}} \cdot \mathrm{a}_{2 \mathrm{k}} \cdot \frac{\mathrm{d}^{\mathrm{k}}}{\mathrm{d} \mathrm{z}^{2 \mathrm{k}}}\left(\frac{\mathrm{m}}{\sqrt{\mathrm{R}^{2}+}-\overline{\mathrm{z}^{2}}}\right), \ldots . . . \text {. . II }\right)
$$

von der beliebig viele Glieder berechnet werden können.

Die Koeffizienten $a_{0}, a_{2}, a_{4} \ldots a_{2 k}$ lassen sich aus 9) nach Ausmultiplizierung der Glieder von J (i $\alpha \mathrm{R})$ mit der angesetzten Potenzreihe durch die Methode der Koeffizientenvergleichung sukzessive berechnen. Man findet so, daß der Anfang der Potenzreihe lautet:

$$
\left.\frac{\mathrm{J}}{\mathrm{J}} \frac{(\mathrm{i} \alpha \mathrm{r})}{\mathrm{i} \alpha \mathrm{R})}=\mathrm{I}-\frac{\mathrm{R}^{2}-\mathrm{r}^{2}}{4} \cdot \alpha^{2}+\frac{\left(\mathrm{R}^{2}-\mathrm{r}^{2}\right)\left(3 \mathrm{R}^{2}-\mathrm{r}^{2}\right)}{64} \cdot \alpha^{4}-\ldots . . . \mathrm{I} 2\right)
$$

Da $r$ der 8) entsprechenden Reihe für $J(i \propto r)$ wegen in allen Koeffizienten $a_{2 k}$ bloß als gerade Potenz vorkommen kann und außerdem: 


$$
\begin{gathered}
-43- \\
\left|\frac{d^{2} k+1}{d z^{2} k+1}\left(\frac{I}{\sqrt{R^{2}+z^{2}}}\right)\right|_{z=0}
\end{gathered}
$$

verschwindet, sind die Randwerte:

$$
\left(\frac{\partial \varphi_{2}}{\partial r}\right)_{r=0} ; \quad\left(\frac{\partial \varphi_{2}}{\partial z}\right)_{z=0}
$$

Null. Die Niveaulinien des Potentials $\varphi_{2}$ verlaufen also ohne Knickung durch die z-Achse und Ebene $(z=0)$ hindurch; auch hat $\varphi_{2}$ als ,analytische" Funktion in seinem ganzen Geltungsbereich [ $\mathrm{r}<\mathrm{R}$ ] sonst stetigen Charakter [vgl. dazu II)].

Bricht man die Entwicklung II) schon nach dem zweiten Gliede ab, so folgt mit Rücksicht auf I2):

$$
\left.\varphi_{2}=-\frac{m}{\sqrt{\mathrm{R}^{2}+\mathrm{z}^{2}}}\left[\mathrm{I}-\frac{\left(\mathrm{R}^{2}-\mathrm{r}^{2}\right)\left(\mathrm{R}^{2}-2 \mathrm{z}^{2}\right)}{4\left(\mathrm{R}^{2}+\mathrm{z}^{2}\right)^{2}}\right] . . . . \quad . \quad \mathrm{I} 3\right)
$$

und für das "Zylinderhohlraumpotential" $p=\varphi_{1}+\varphi_{2}$ :

$$
\left.\varphi=\frac{\mathrm{m}}{\sqrt{\mathrm{r}^{2}+\mathrm{z}^{2}}}-\frac{\mathrm{m}}{\sqrt{\mathrm{R}^{2}+\mathrm{z}^{2}}}\left[\mathrm{I}-\frac{\left(\mathrm{R}^{2}-\mathrm{r}^{2}\right)\left(\mathrm{R}^{2}-2 \mathrm{z}^{2}\right)}{4\left(\mathrm{R}^{2}+\mathrm{z}^{2}\right)^{2}}\right] . . . . \quad \mathrm{I} 4\right)
$$

Hieraus läßt sich die elektrische Dichte $\eta$ der Zylinderfläche bestimmen durch:

Man erhält so:

$$
\eta=\frac{\mathrm{I}}{4 \pi}\left(\frac{\partial \varphi}{\partial \mathrm{r}}\right)_{\mathrm{r}=\mathrm{R}}
$$

$$
\left.\eta(\mathrm{z})=-\frac{3}{8} \cdot \frac{\mathrm{m}}{\pi} \cdot \frac{\mathrm{R}^{3}}{\left(\mathrm{R}^{2}+\mathrm{z}^{2}\right)^{5 / 2}}, \quad \cdot \mathrm{I} 5\right)
$$

und durch Integration über die ganze Zylinderfläche (Z) folgt:

$$
q=\int_{(\mathrm{z})} \eta \mathrm{d} \sigma=\int_{-\infty}^{+\infty} \int_{0}^{2 \pi} \eta(\mathrm{z}) \cdot \mathrm{Rdzd} \gamma=-\mathrm{m},
$$

d. h. auch die Näherungslösung zeigt schon, wie es notwendigerweise sein muB, wenn $\varphi$ wie im vorliegenden Falle im Zylinderaußenraum Null ist, daB die am Zylinder induzierte Menge entgegengesetzt gleich der Menge des Poles $(+\mathrm{m})$ ist.

Die zur Auswertung des Integrals 7$)$ verwendete Methode der Reihenentwicklung führt, wie leicht einzusehen ist, auch dann noch zum Ziele, wenn an Stelle der Randfunktion:

$$
\frac{m}{\sqrt{R^{2}+z^{2}}}
$$

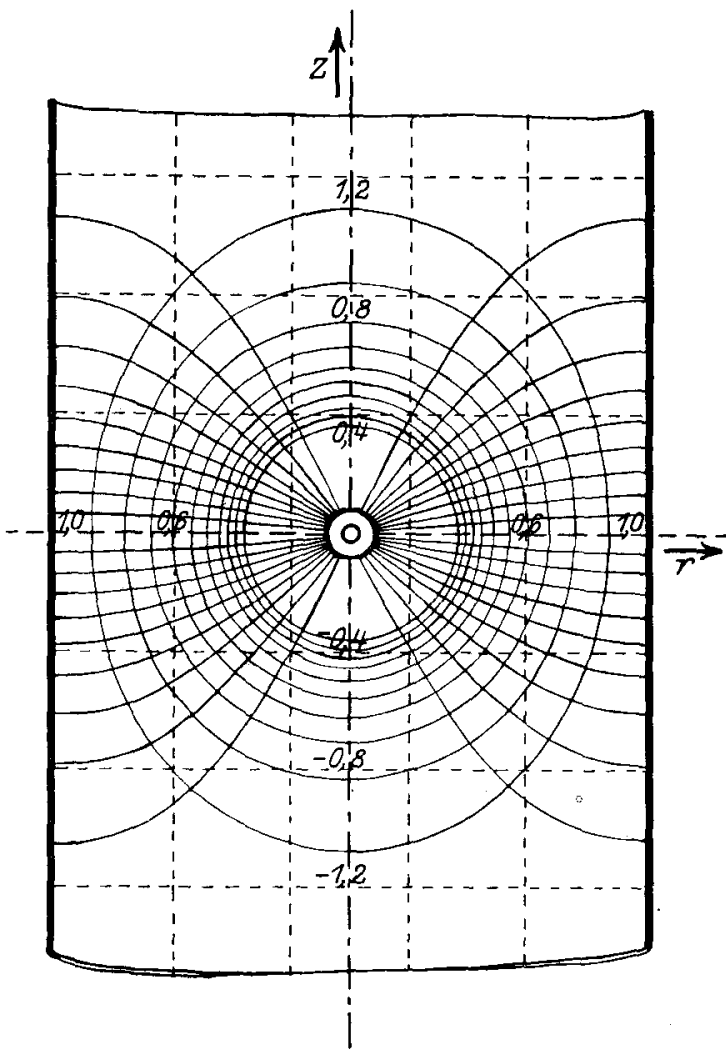

Abb. 17.

der Zylinderfläche irgendeine andere willkürliche Funktion $F(z)$ tritt, die bloB der folgenden Bedingungsgleichung, in welcher $\mathrm{k}$ irgendeine positive ganze Zahl bedeutet, zu genügen hat:

$$
\left.\lim _{\alpha=\infty}\left(\alpha^{2 \mathrm{k}} \int_{-\infty}^{+\infty} \mathrm{F}(\lambda) \mathrm{e}^{\mathrm{i} \alpha \lambda} \cdot \mathrm{d} \lambda\right) \equiv 0^{1}\right) .
$$

1) Diese Bedingungsgleichung ergibt sich unter der Voraussetzung, daf in Gleichung 3) die 
Das Potential $\varphi^{(1)}$ [Formel I2) des vorhergehenden Abschnittes] ist deshalb mathematisch besonders interessant, weil es sich, abgesehen von der erwähnten Konvergenzbedingung, sonst für eine willkürliche Randfunktion durch eine der Formel II) dieses Abschnittes entsprechende Reihe ausdrücken läßt, von der beliebig viele Glieder auf elementare Weise berechenbar sind.

Anwendung der Potentiallösung des Zylinder-Hohlraumpotentials auf das konzentrische Kabel. Zum Schlusse sei noch bemerkt, daß sich die mathematische Lösung des Zylinder-Hohlraumpotentials zur Bestimmung der Potentialverteilung im Isoliermantel des gestreckten, konzentrischen Kabels verwenden läßt, sofern nur der Isoliermantel aus homogenem Material besteht und die Kabelader im Vergleich zu dessen Durchmesser dünn ist. Dann ist es zulässig, die Kabelader als linear und also als Superposition von Massenpolen aufufassen. Die auf den äußern Begrenzungszylinder der Kabelisolation bezogenen Zylinder-Hohlraumpotentiale aller dieser Massenpole liefern in ihrer Summe ein Potential, das sich, falls die Massenverteilung stetig ist, dort verhält wie das Potential eines linearen Leiters (vgl. darüber S. 23) und an der Zylinderfläche verschwindet. Das Potential $\varphi$ des Kabelinnern sei gegeben durch die Randwerte

$$
\left.\Pi=2 \lambda(\mathrm{z}) \cdot \lg \frac{\mathrm{I}}{\varrho} \ldots . . . . . . . \quad \mathrm{I} 6\right)
$$

$[\lambda(z)=$ lineare Dichte (willkürliche Funktion); $2 \varrho=$ Leiterdicke $]$

des in der z-Achse liegenden linearen Leiters und wird also nach I) und 7) dargestellt durch:

$$
\varphi=\int_{(\mathbf{L})} \frac{\lambda(\zeta)}{\sqrt{r^{2}+(\mathbf{z}-\zeta)^{2}}} \mathrm{~d} \zeta-\int_{(\mathbf{L})} \lambda(\zeta) \mathrm{d} \zeta \int_{0}^{\infty} \cos \alpha(z-\zeta) \cdot \frac{\mathrm{J}(\mathrm{i} \alpha \mathrm{r})}{\mathrm{J}(\mathrm{i} \alpha \mathrm{R})} \cdot \mathrm{H}(\mathrm{i} \alpha \mathrm{R}) \mathrm{d} \alpha
$$

Reihenentwicklung I2) für $\frac{\mathrm{J}(\mathrm{i} \alpha \mathrm{r})}{\mathrm{J}(\mathrm{i} \alpha \mathrm{R})}$ verwendet und die Integration dann gliedweise ausgeführt wird, aus einem Satze uber „bedingt" konvergierende Integrale. [Vgl. dazu: Riemann-Weber, Part. Diff.Gl., 5. Aufl. Bd. I, Seite 17 oben.] 
Anhang.

$-$ 


\section{$-4^{6}-$ \\ Konstruktion der Kraftlinienbilder.}

Alle in dieser Schrift gezeichneten Kraftlinienbilder sind Meridianschnitte von axialsymmetrischen Feldern. Überall wurden Zylinderkoordinaten ( $r, z)$ mit der z-Achse als Symmetrieachse verwendet.

Zur Konstruktion der Bilder denken wir uns das Potential $\varphi$ als dritte, rechtwinklige Koordinate über einer Meridianebene [Tafel $(r, z)]$ aufgetragen. $\varphi$ wird dann dargestellt durch 'eine in bestimmter Weise verlaufende Fläche [Potentialfläche], aus welcher die Niveaulinien als Schnitte mit den Ebenen $\varphi=$ konst. hervorgehen. Die Potentialfäche ist für die Bilder festgelegt durch zwei Kurvenscharen, welche in zwei zueinander senkrecht stehenden Parallelebenen-Büscheln liegen. Die Achsen dieser Büschel sind die Tafelnormalen in den unendlich fernen Punkten der $r$ - und $z$-Achsen. - Die Niveaulinien der Abbildungen besitzen je gleiche Potentialunterschiede; sie sind daher hervorgegangen aus den Schnittkurven eines Systems äquidistanter Hauptebenen mit der Potentialfäche.

Für die Kraftlinien (Begrenzungen der Kraftröhrenschnitte) wurden die Anfänge und Enden an den Leitern derart ermittelt, daß die Kraftröhren dort je gleiche Elektrizitätsmengen überdecken und somit für jede beliebige Querschnittsfläche auch denselben Kraftfluß besitzen. Von der Konstruktion weiterer Punkte wurde abgesehen; die Kraftlinien sind hernach einfach unter möglichster Berücksichtigung des rechtwinkligen Schnittes mit den Niveaulinien zwischen Anfang und Ende eingezeichnet worden.

Während also die Niveaulinien vollständig einwandsfrei konstruiert sind, gilt dies für die Kraftlinien in Anbetracht der Konstruktionsweise nur mehr oder weniger.

\section{Influenzwirkung eines elektrischen Poles auf die Kreislochscheibe.}

Formel 6):

(Vergleiche Abschnitt III.)

$$
p=\frac{2}{\pi} \cdot \frac{\mathrm{m}}{\sqrt{\mathrm{r}^{2}}+\mathrm{z}^{2}} \cdot \arccos \frac{\sqrt{\mathrm{a}^{2} \mathrm{z}^{2}+\left(\mathrm{r}^{2}+\mathrm{z}^{2}+\mathrm{ar}\right)^{2}}-\sqrt{\mathrm{a}^{2} \mathrm{z}^{2}+\left(\mathrm{r}^{2}+\mathrm{z}^{2}-\mathrm{ar}\right)^{2}}}{2 \mathrm{ar}} .
$$

Tabelle für $\varphi(a=1, m=r):$

\begin{tabular}{|c|c|c|c|c|c|c|c|c|}
\hline \multirow{2}{*}{$z$} & \multicolumn{8}{|c|}{$\mathbf{r}$} \\
\hline & 0 & 0,25 & $0,5^{\circ}$ & 0,75 & $1, O$ & $\mathrm{I}, 5$ & 2,0 & 2,5 \\
\hline 0 & $\infty$ & 3,3333 & $\mathrm{I}, 33^{\circ} 3$ & 0,6 I 43 & 0 & $o$ & 0 & o \\
\hline 0,25 & 3,3762 & 2,1992 & $I, 1426$ & 0,5876 & 0,2589 & 0,05876 & 0,02313 & 0,01087 \\
\hline 0,50 & 1,4096 & 1,1960 & 0,8144 & 0,5021 & 0,2902 & 0,09716 & 0,04083 & 0,02077 \\
\hline 1,0 & 0,50000 & 0,4709 & 0,3979 & 0,3097 & 0,2284 & 0, I I 64 & $0,0607 \mathrm{I}$ & 0,03446 \\
\hline 1,5 & 0,24956 & 0,2417 & 0,2202 & o, I 904 & $0,158 \mathrm{I}$ & 0, IOI 4 & 0,06299 & 0,03976 \\
\hline 2,0 & $0, \mathbf{I} 475^{8}$ & 0,1447 & $0,13^{6} 5$ & 0,1244 & 0, I IOI & 0,08087 & 0,05679 & 0,03942 \\
\hline 3,0 & $0,0 \circ 8278$ & 0,06764 & $0,065^{8}$ & 0,0469 & - & - & - & 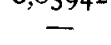 \\
\hline
\end{tabular}


Formel ro):

$$
\mathfrak{q}(\mathrm{r})=-\frac{2}{\pi} \mathrm{m} \cdot \arccos \left(\frac{\mathrm{a}}{\mathrm{r}}\right)
$$

(Berechnung der Tabellen mittelst fünfstelliger Logarithmen.)

Tabelle für $\mathfrak{q}(a=I$, $\mathrm{m}=\mathrm{I})$ :

\begin{tabular}{c|c}
\hline$r$ & $-q(\mathbf{r})$ \\
\hline $\mathbf{1}, 0$ & 0 \\
$\mathbf{1 , 2}$ & 0,3729 \\
$\mathbf{1 , 4}$ & 0,49349 \\
$\mathbf{1 , 6}$ & 0,57019 \\
$\mathbf{1 , 8}$ & 0,62500 \\
2,0 & 0,66666 \\
2,2 & 0,69959 \\
2,4 & 0,72639 \\
2,6 & 0,74867 \\
$\infty$ & 1
\end{tabular}

Zugehörige Zeichnungen findet man nebenstehend und auf Seite II.
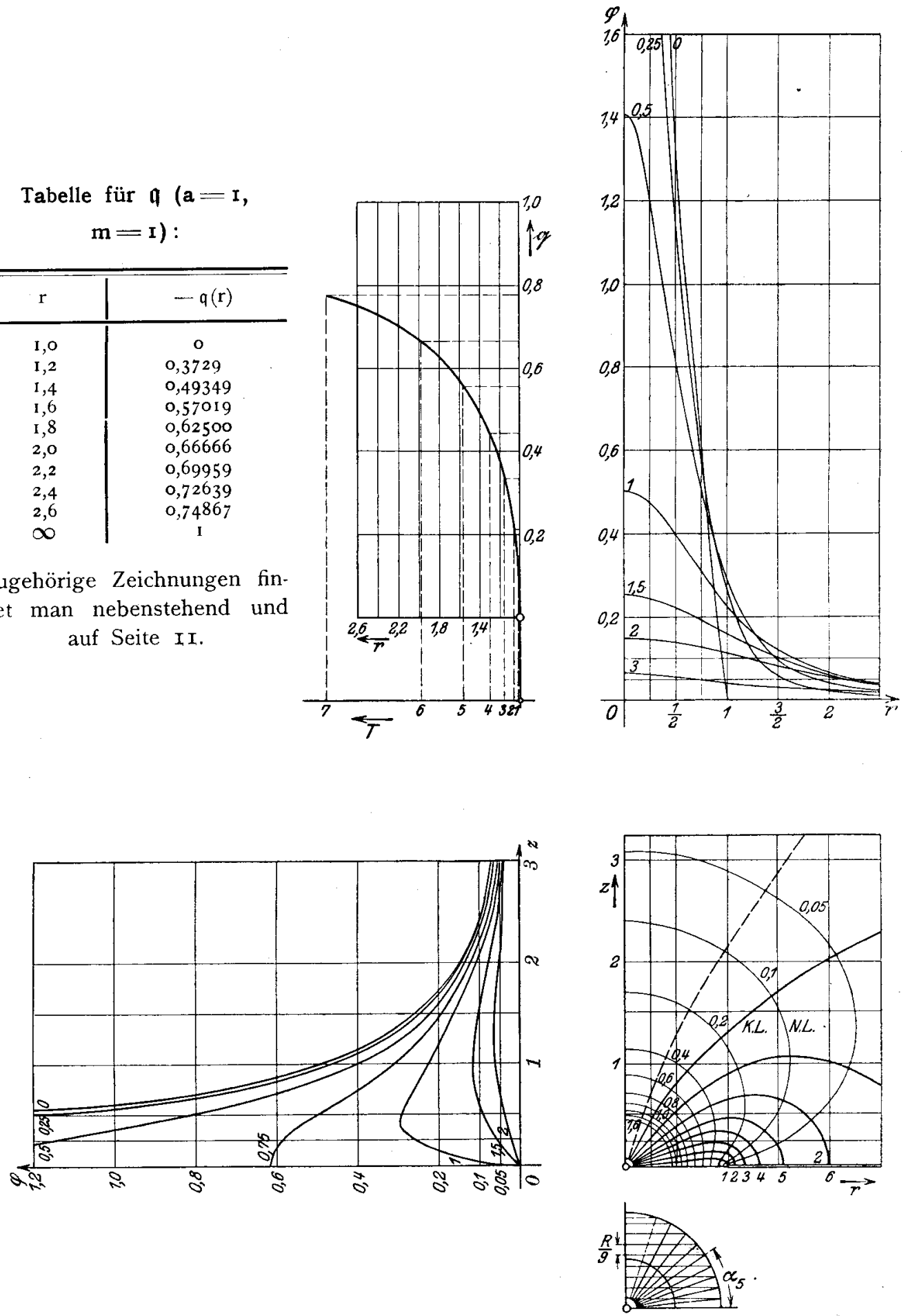


\section{Stab-Ring-Potential.}

Nach Formel 3) ist:

(Vergleiche Abschnitt VI, A.)

$\varphi_{1}=\frac{m}{2 h} \cdot \lg \left\{\left[\frac{\left.h+z+\sqrt{(h+z)^{2}+r^{2}}\right]\left[h-z+\sqrt{(h-z)^{2}+r^{2}}\right]}{r^{2}}\right\}\right.$, für $0<z<h$,

$\varphi_{1}=\frac{\mathrm{m}}{2 \mathrm{~h}} \cdot \lg \left[\frac{\mathrm{z}+\mathrm{h}+\sqrt{(\mathrm{z}+\mathrm{h})^{2}+\mathrm{r}^{2}}}{\mathrm{z}-\mathrm{h}+\sqrt{(\mathrm{z}-\mathrm{h})^{2}+\mathrm{r}^{2}}}\right]$, für $\mathrm{z}>\mathrm{h}$,

und nach Formel 7):

$\varphi_{2}=-\frac{2}{\pi} \cdot \frac{\mathrm{m}}{\sqrt{(\mathrm{R}+\mathrm{r})^{2}+\mathrm{z}^{2}}} \int_{0}^{\overline{2}} \frac{\mathrm{d} \xi}{\sqrt{\mathrm{I}-\mathrm{k}^{2}}} ; \quad \mathrm{\operatorname {sin } ^ { 2 } \xi}=2 \sqrt{\frac{\mathrm{Rr}}{(\mathrm{R}+\mathrm{r})^{2}+\mathrm{z}^{2}}} ; \quad \varphi=\varphi_{1}+\varphi_{2}$.

(Das elliptische Vollintegral in $\varphi_{2}$ wurde nach der Funktionentafel von Jahnke und Emde abgelesen.)

Tabelle für $\varphi_{1}, \varphi_{2}$ und $\varphi$, wenn $\mathrm{m}=\mathrm{I}, \mathrm{a}=\mathrm{I}$ und $\mathrm{h}=\mathbf{2}$ :

\begin{tabular}{|c|c|c|c|c|c|c|c|c|c|c|}
\hline \multirow{2}{*}{$z$} & \multirow{2}{*}{$\begin{array}{c}\varphi= \\
\varphi_{1}+\varphi_{2}\end{array}$} & \multicolumn{9}{|c|}{$r$} \\
\hline & & 0,0 & 0,125 & 0,25 & $0,5^{\circ}$ & 0,75 & $\mathrm{I}, \mathrm{OO}$ & 1,25 & I, 5 & 2,0 \\
\hline o & $\begin{array}{l}\varphi_{1} \\
\varphi_{2} \\
\varphi\end{array}$ & $\frac{\infty}{\infty}$ & $\begin{array}{r}1,7333 \\
-1,0039 \\
+0,7294\end{array}$ & $\begin{array}{r}1,3882 \\
-1,0164 \\
+0,3718\end{array}$ & $\begin{array}{r}1,0474 \\
-1,0732 \\
-0,0258\end{array}$ & $\begin{array}{r}0,8537 \\
-1,2164 \\
-0,3627\end{array}$ & $\begin{array}{c}0,7218 \\
-\quad \infty \\
-\quad \infty\end{array}$ & $\begin{array}{r}0,6245 \\
-1,0165 \\
-0,3920\end{array}$ & $\begin{array}{r}0,5493 \\
-0,7681 \\
-0,2188\end{array}$ & $\begin{array}{r}0,4407 \\
-0,4306 \\
+0,0101\end{array}$ \\
\hline 0,25 & $\begin{array}{l}\varphi_{1} \\
\varphi_{2} \\
\varphi\end{array}$ & $\frac{\infty}{\infty}$ & $\begin{array}{r}1,7294 \\
-0,9732 \\
+0,7562\end{array}$ & $\begin{array}{r}1,3846 \\
-0,982 I \\
+0,4025\end{array}$ & $\begin{array}{r}1,0438 \\
-1,0205 \\
+0,0233\end{array}$ & $\begin{array}{r}0,8505 \\
-1,0863 \\
-0,2358\end{array}$ & $\begin{array}{r}0,7190 \\
-1,0998 \\
-0,3808\end{array}$ & $\begin{array}{r}0,6221 \\
-0,8638 \\
-0,2417\end{array}$ & $\begin{array}{r}0,5473 \\
-0,7383 \\
-0,1910\end{array}$ & $\begin{array}{r}0,4393 \\
-0,5295 \\
-0,0902\end{array}$ \\
\hline $0,5^{\circ}$ & $\begin{array}{l}\varphi_{1} \\
\varphi_{2} \\
\varphi\end{array}$ & $\frac{\infty}{\infty}$ & $\begin{array}{r}1,7173 \\
-0,8971 \\
+0,8202\end{array}$ & $\begin{array}{r}1,3725 \\
-0,9858 \\
+0,3867\end{array}$ & $\begin{array}{r}1,0327 \\
-0,9087 \\
+0,1240\end{array}$ & $\begin{array}{r}0,8406 \\
-0,9115 \\
-0,0708\end{array}$ & $\begin{array}{r}0,7105 \\
-0,874 \mathrm{1} \\
-0,1636\end{array}$ & $\begin{array}{r}0,6149 \\
-0,7818 \\
-0,1669\end{array}$ & $\begin{array}{r}0,54 \mathrm{I} 3 \\
-0,6748 \\
-0, \mathrm{I} 335\end{array}$ & $\begin{array}{r}0,435^{2} \\
-0,5102 \\
-0,075^{\circ}\end{array}$ \\
\hline 0,75 & $\begin{array}{l}\varphi_{1} \\
\varphi_{2} \\
\varphi\end{array}$ & $\frac{\infty}{\infty}$ & $\begin{array}{r}1,6957 \\
-0,7998 \\
+0,8959\end{array}$ & $\begin{array}{r}1,3510 \\
-0,7992 \\
+0,5518\end{array}$ & $\begin{array}{r}1,0133 \\
-0,7942 \\
+0,2191\end{array}$ & $\begin{array}{r}0,8236 \\
-0,7775 \\
+0,0461\end{array}$ & $\begin{array}{r}0,6960 \\
-0,7393 \\
-0,0433\end{array}$ & $\begin{array}{r}0,6045 \\
-0,6788 \\
-0,0743\end{array}$ & $\begin{array}{r}0,5313 \\
-0,6088 \\
-0,0775\end{array}$ & $\begin{array}{r}0,4125 \\
-0,4838 \\
-0,07 \times 3\end{array}$ \\
\hline 1,25 & $\begin{array}{l}\varphi_{1} \\
\varphi_{2} \\
\varphi\end{array}$ & $\frac{\infty}{\infty}$ & $\begin{array}{r}1,6108 \\
-0,6240 \\
+0,9868\end{array}$ & $\begin{array}{r}1,2695 \\
-0,62 I 5 \\
+0,6480\end{array}$ & $\begin{array}{r}0,94 \mathrm{I} 4 \\
-0,6 \mathrm{r} \mathbf{6} 6 \\
+0,3298\end{array}$ & $\begin{array}{r}0,7635 \\
-0,5939 \\
+0,1696\end{array}$ & $\begin{array}{r}0,6469 \\
-0,5681 \\
+0,0788\end{array}$ & $\begin{array}{r}0,563 \mathrm{I} \\
-0,5356 \\
+0,0275\end{array}$ & $\begin{array}{r}0,4993 \\
-0,4988 \\
+0,0005\end{array}$ & $\begin{array}{r}0,4072 \\
-0,4251 \\
-0,0179\end{array}$ \\
\hline 1,75 & $\begin{array}{l}\varphi_{1} \\
\varphi_{2} \\
\varphi\end{array}$ & $\frac{\infty}{\infty}$ & $\begin{array}{r}\mathrm{I}, 3846 \\
-0,4955 \\
+0,889 \mathrm{I}\end{array}$ & $\begin{array}{r}1,0709 \\
-0,4938 \\
+0,5771\end{array}$ & $\begin{array}{r}0,7984 \\
-0,4866 \\
+0,3 \text { I } 18\end{array}$ & $\begin{array}{r}0,6590 \\
-0,4748 \\
+0,1842\end{array}$ & $\begin{array}{r}0,5700 \\
-0,4587 \\
+0,1113\end{array}$ & $\begin{array}{r}0,5043 \\
-0,4392 \\
+0,0651\end{array}$ & $\begin{array}{r}0,4533 \\
-0,4 \text { I } 74 \\
+0,0359\end{array}$ & $\begin{array}{r}0,3778 \\
-0,3710 \\
+0,0068\end{array}$ \\
\hline 2,25 & $\begin{array}{l}\varphi_{1} \\
\varphi_{2} \\
\varphi\end{array}$ & $\begin{array}{r}0,6922 \\
-0,406 \mathrm{I} \\
+0,2860\end{array}$ & $\begin{array}{r}0,6940 \\
-0,4058 \\
+0,2882\end{array}$ & $\begin{array}{r}0,6615 \\
-0,4045 \\
+0,2570\end{array}$ & $\mid \begin{array}{r}0,5889 \\
-0,3999 \\
+0,1890\end{array}$ & $\begin{array}{r}0,5270 \\
-0,3924 \\
+0,1346\end{array}$ & $\begin{array}{r}0,4765 \\
-0,3822 \\
+0,0943\end{array}$ & $\begin{array}{r}0,4348 \\
-0,3700 \\
+0,0648\end{array}$ & $\begin{array}{r}0,3996 \\
-0,3560 \\
+0,0436\end{array}$ & $\begin{array}{r}0,3434 \\
-0,3261 \\
+0,0173\end{array}$ \\
\hline 2,75 & $\begin{array}{l}\varphi_{1} \\
\varphi_{2} \\
\varphi\end{array}$ & $\begin{array}{r}0,4614 \\
-0,3417 \\
+0,1197\end{array}$ & $\begin{array}{r}0,4598 \\
-0,3415 \\
+0,1183\end{array}$ & $\begin{array}{r}0,4549 \\
-0,3407 \\
+0, \text { I I } 2\end{array}$ & $\begin{array}{r}0,4381 \\
-0,3377 \\
+0,1004\end{array}$ & $\begin{array}{r}0,4 \text { I } 59 \\
-0,3328 \\
+0,083 \mathrm{I}\end{array}$ & $\begin{array}{r}0,3923 \\
-0,3261 \\
+0,0662\end{array}$ & $\begin{array}{r}0,3691 \\
-0,3173 \\
+0,0518\end{array}$ & $\begin{array}{r}0,3472 \\
-0,3087 \\
+0,0385\end{array}$ & $\begin{array}{r}0,3084 \\
-0,2879 \\
+0,0205\end{array}$ \\
\hline
\end{tabular}

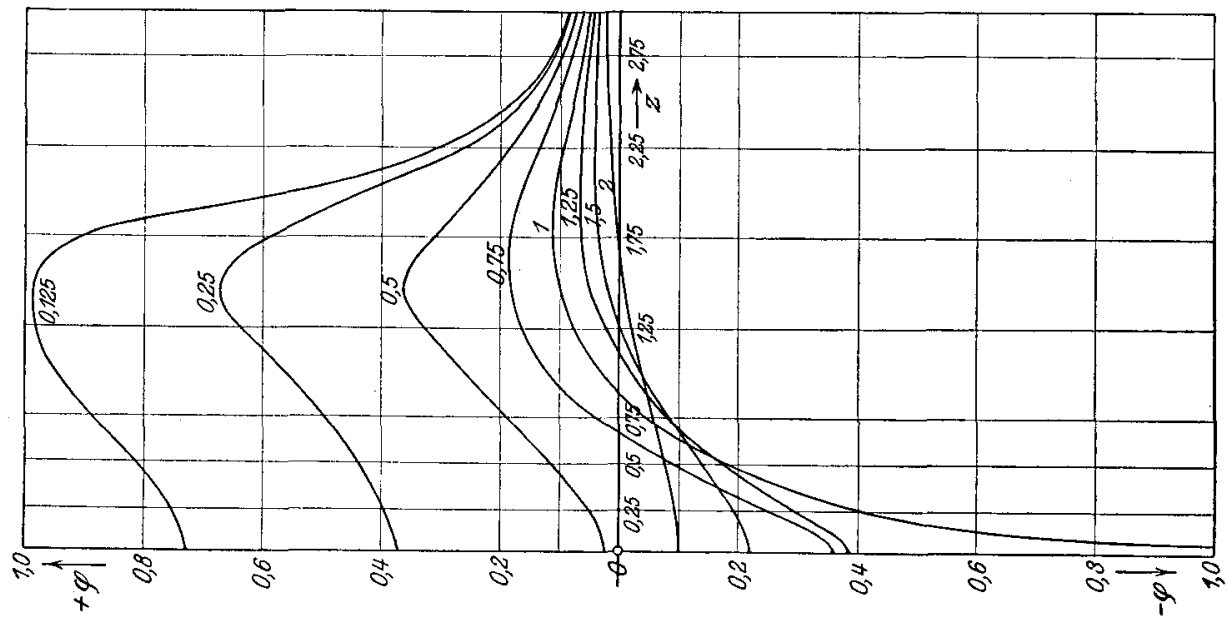


Die Konstruktionszeichnungen, welche zum Kraftlinienbilde des Stab-Ring-Potentiales (vergleiche Abb. 9, Seite 28) gehören, befinden sich im Anhange auf den Seiten 48 und 49 .

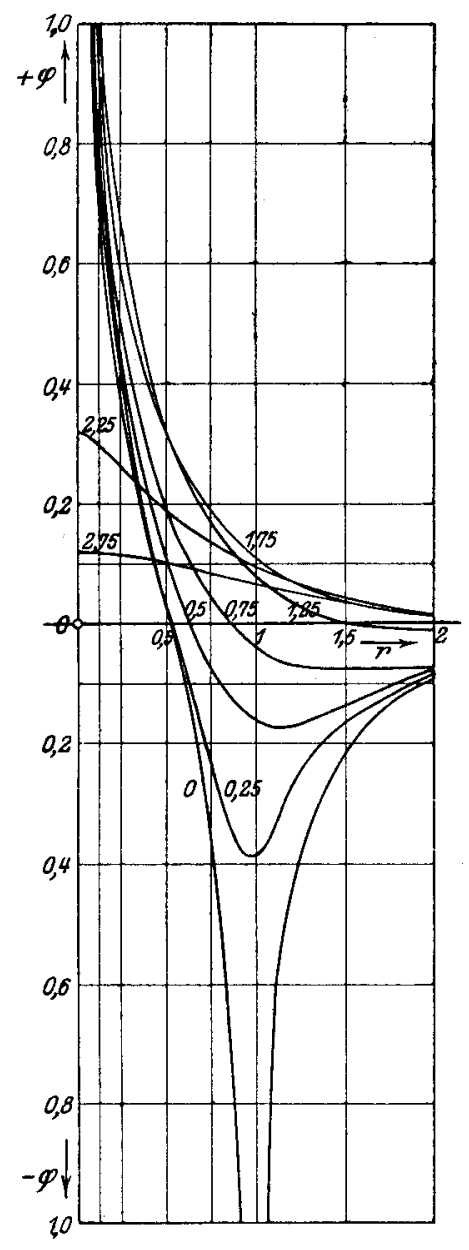

\section{Isolatorproblem.}

Zum Bilde S. 33, Abschnitt VII.

Die Kraft- und Niveaulinien sind dort Scharen zueinander konfokaler Ellipsen und Hyperbeln [Brennpunkte in $\mathrm{F}_{1}(\mathrm{r}=-\mathrm{a}, \mathrm{z}=0)$ und $\left.\mathrm{F}_{2}(\mathrm{r}=+\mathrm{a}, \mathrm{z}=0)\right]$. Die Kraftlinien sind durch ihre Anfänge am linearen Leiter bestimmt. Besitzen alle Kraftröhren die gleiche Anzahl Einheitslinien, so sind die Anfangspunkte ihrer Begrenzungskraftlinien in irgendeinem Meridianschnitt je gleich weit voneinander entfernt. Die Niveaulinien können festgelegt werden durch Angabe ihrer Punkte an der Grenze $z=0$. Gleiche Potentialdifferenzen erzielt man zwischen benachbarten Niveaulinien durch Berechnung der Bestimmungspunkte $(\mathrm{r}, \mathrm{z}=0)$ mit der aus Formel II) von Abschnitt VII hergeleiteten Gleichung:

$$
\mathrm{r}=\frac{\mathrm{a}}{\mathfrak{E} \mathfrak{v}\left[\frac{\mathrm{a}}{\mathrm{m}} \cdot(\varphi)_{\mathrm{z}=0}\right]},
$$

wenn man für $(\varphi)_{\mathbf{z}=0}$ die entsprechenden Potentialwerte einführt.

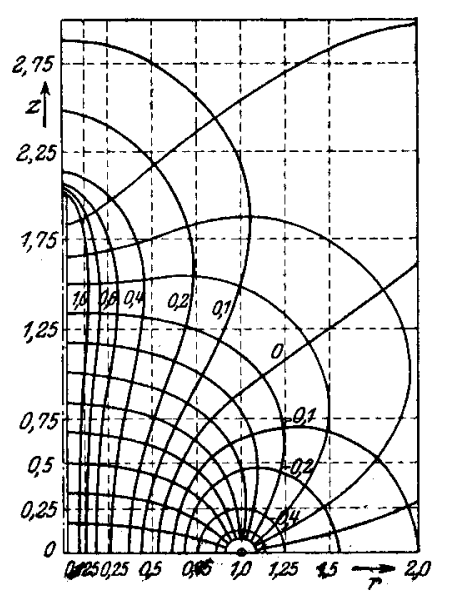

Tabelle für $\mathrm{r}(\mathrm{a}=\mathrm{I}, \mathrm{m}=\mathrm{I})$ :

\begin{tabular}{c|c}
\hline$\varphi$ & $\mathbf{r}$ \\
\hline 0 & $\mathbf{1}, 0000$ \\
0,2 & 0,9804 \\
0,4 & 0,9465 \\
0,6 & 0,8435 \\
0,8 & 0,7476 \\
1,0 & 0,6480 \\
1,2 & 0,5523 \\
1,6 & 0,3880 \\
2,0 & 0,2658 \\
2,4 & 0,1800
\end{tabular}

Nach diesen Angaben kann das ganze Kraftlinienbild mittels planimetrischer Konstruktionen gezeichnet werden. 


\section{Zum Kraftlinienbilde S. 37 von Abschnitt VII:}

Das Potential $\varphi$ der Formel 33), Abschn. VII setzt sich zusammen aus dem Potentiale $\varphi_{1}$, das auf S. 48 durch Formel und tabellarische Werte angegeben ist, und einer Potentialfunktion $\varphi_{2}$ :

$$
\begin{gathered}
\varphi_{2}=-\frac{2}{\pi} \cdot \frac{\mathrm{m}}{\mathrm{h}} \cdot \Re \int_{\mathrm{a}}^{\infty} \frac{\operatorname{arctg} \frac{\mathrm{h}}{\xi}}{\sqrt{(\xi+\mathrm{iz})^{2}-\mathrm{r}^{2}}} \mathrm{~d} \xi=-\frac{2}{\pi} \cdot \frac{\mathrm{m}}{\mathrm{h}} \mathfrak{\Re} \int_{0}^{\frac{1}{\mathrm{a}}} \frac{\operatorname{arctg}(\eta \mathrm{h})}{\sqrt{(\mathrm{I}+\mathrm{i} \eta \mathrm{z})^{2} \cdot \ldots(\eta \mathrm{r})^{2}}} \cdot \frac{\mathrm{d} \eta}{\eta} \\
\text { (Substitution } \left.\eta=\frac{\mathrm{I}}{\xi}\right)
\end{gathered}
$$

woraus der Realteil:

folgt, wo:

$$
\left.\varphi_{2}=-\frac{2}{\pi} \cdot \frac{\mathrm{m}}{\mathrm{h}} \int_{0}^{\frac{1}{\mathrm{a}}} \operatorname{arctg}(\eta \mathrm{h}) \cdot \sqrt{\frac{\mathrm{x}+\sqrt{\mathrm{x}^{2}+\mathrm{y}^{2}}}{2\left(\mathrm{x}^{2}+\mathrm{y}^{2}\right)}} \cdot \frac{\mathrm{d} \eta}{\eta} . . . . . . \mathrm{I}\right)
$$

$$
\mathrm{x}=\mathrm{x}-\eta^{2}\left(\mathrm{r}^{2}+\mathrm{z}^{2}\right) ; \quad \mathrm{y}=2 \eta \mathrm{z} \quad \text { ist. }
$$

Da der Integrand von $\varphi_{2}$ Gl. I) in den Grenzen überall endlich bleibt, ist dieselbe zur näherungsweisen Berechnung von $\varphi_{2}$ geeignet. Für die Werte von $\varphi_{2}$ in der nach-

\begin{tabular}{|c|c|c|c|c|c|c|c|c|}
\hline \multirow{2}{*}{$\boldsymbol{z}$} & \multirow{2}{*}{$\varphi$} & \multicolumn{7}{|c|}{$\mathbf{r}$} \\
\hline & & $\circ$ & 0,125 & 0,25 & 0,50 & 0,75 & $\mathrm{I}, \mathrm{O}$ & 1,5 \\
\hline 0 & $\begin{array}{l}\varphi_{1} \\
\varphi_{8} \\
\varphi .\end{array}$ & $\frac{\infty}{\infty}$ & $\begin{array}{r}\mathrm{I}, 7333 \\
-0,5028 \\
\mathrm{I}, 2305\end{array}$ & $\begin{array}{r}1,3882 \\
-0,5060 \\
0,8822\end{array}$ & $\begin{array}{r}\mathrm{I}, 0474 \\
-0,52 \mathrm{I} 3 \\
0,526 \mathrm{I}\end{array}$ & $\begin{array}{r}0,8537 \\
-0,5592 \\
0,2945\end{array}$ & $\begin{array}{c}0,7218 \\
-0,7218 \\
0\end{array}$ & $\begin{array}{c}0,5493 \\
-0,5493 \\
0\end{array}$ \\
\hline 0,25 & $\begin{array}{l}\varphi_{1} \\
\varphi_{2} \\
\varphi\end{array}$ & $\frac{\infty}{\infty}$ & $\begin{array}{r}1,7294 \\
-0,4938 \\
1,2356\end{array}$ & $\begin{array}{r}\mathbf{I}, 3846 \\
-\mathbf{0 , 4 9 6 6} \\
\mathbf{0 , 8 8 8 0}\end{array}$ & $\begin{array}{r}1,0438 \\
-0,5119 \\
0,5319\end{array}$ & $\begin{array}{r}0,8505 \\
-0,5288 \\
0,3217\end{array}$ & $\begin{array}{r}0,7190 \\
-0,5505 \\
0,1686\end{array}$ & $\begin{array}{r}0,5473 \\
-0,4924 \\
0,0549\end{array}$ \\
\hline 0,75 & $\begin{array}{l}\varphi_{1} \\
\varphi_{2} \\
\varphi\end{array}$ & $\frac{\infty}{\infty}$ & $\begin{array}{r}1,6957 \\
-0,4395 \\
1,2562\end{array}$ & $\begin{array}{r}1,3510 \\
-0,4409 \\
0,9101\end{array}$ & $\begin{array}{r}1,0133 \\
-0,4416 \\
0,57 \times 7\end{array}$ & $\begin{array}{r}0,8236 \\
-0,4407 \\
0,3829\end{array}$ & $\begin{array}{r}0,6960 \\
-0,4285 \\
0,2675\end{array}$ & $\begin{array}{r}0,5313 \\
-0,4028 \\
0,1285\end{array}$ \\
\hline 1,25 & $\begin{array}{l}\varphi_{1} \\
\varphi_{2} \\
\varphi\end{array}$ & $\frac{\infty}{\infty}$ & $\begin{array}{r}1,6108 \\
-0,3769 \\
1,2339\end{array}$ & $\begin{array}{r}1,2695 \\
-0,3763 \\
0,893^{2}\end{array}$ & $\begin{array}{r}0,9414 \\
-0,3743 \\
0,567 \mathrm{I}\end{array}$ & $\begin{array}{r}0,7635 \\
-0,3701 \\
0,3934\end{array}$ & $\begin{array}{r}0,6470 \\
-0,3636 \\
0,2834\end{array}$ & $\begin{array}{r}0,4993 \\
-0,3423 \\
0,1570\end{array}$ \\
\hline $\mathrm{I}, 75$ & $\begin{array}{l}\varphi_{1} \\
\varphi_{2} \\
\varphi\end{array}$ & $\frac{\infty}{\infty}$ & $\begin{array}{r}\mathrm{I}, 3846 \\
-0,3306 \\
\mathrm{I}, 054^{\circ}\end{array}$ & $\begin{array}{r}1,0709 \\
-0,3230 \\
0,7479\end{array}$ & $\begin{array}{r}0,7984 \\
-0,3209 \\
0,4775\end{array}$ & $\begin{array}{r}0,6590 \\
-0,3173 \\
0,3417\end{array}$ & $\begin{array}{r}0,5700 \\
-0,3121 \\
0,2579\end{array}$ & $\begin{array}{r}0,4533 \\
-0,2975 \\
0,1558\end{array}$ \\
\hline 2,25 & $\begin{array}{l}\varphi_{1} \\
\varphi_{2} \\
\varphi\end{array}$ & $\begin{array}{r}0,7083 \\
-0,2815 \\
0,4268\end{array}$ & $\begin{array}{r}0,6940 \\
-0,2813 \\
0,4127\end{array}$ & $\begin{array}{r}0,66 \text { I5 } \\
-0,2780 \\
0,3835\end{array}$ & $\begin{array}{r}0,5889 \\
-0,2792 \\
0,3097\end{array}$ & $\begin{array}{r}0,5270 \\
-0,2765 \\
0,2515\end{array}$ & $\begin{array}{r}0,4765 \\
-0,2746 \\
0,2019\end{array}$ & $\begin{array}{r}0,3996 \\
-0,2622 \\
0,1374\end{array}$ \\
\hline 2,75 & $\begin{array}{l}\varphi_{1} \\
\varphi_{2} \\
\varphi\end{array}$ & $\begin{array}{r}0,4615 \\
-0,248 \mathrm{r} \\
0,2134\end{array}$ & $\begin{array}{r}0,4598 \\
-0,2480 \\
0,2118\end{array}$ & $\begin{array}{r}0,4549 \\
-0,2477 \\
0,2072\end{array}$ & $\begin{array}{r}0,4381 \\
-0,2465 \\
0,1916\end{array}$ & $\begin{array}{r}0,4159 \\
-0,2444 \\
0,1715\end{array}$ & $\begin{array}{r}0,3923 \\
-0,2416 \\
0,1507\end{array}$ & $\begin{array}{r}0,3472 \\
-0,2339 \\
0,1133\end{array}$ \\
\hline
\end{tabular}
folgenden Tabelle wurde die Simpsonsche Regel verwendet und dabei das Integrationsintervall in ro gleiche Teile zerlegt.

Tabelle für $\varphi_{1}, \varphi_{2}$ und $\varphi=\varphi_{1}+\varphi_{2}(\mathrm{a}=\mathrm{I}, \mathrm{m}=\mathrm{I}, \mathrm{h}=2)$ :

Nach den Daten der Tabelle entworfene Abbildungen siehe Abschnitt VII, S. 37 und auch S. 5I.

In der nachfolgenden Tabelle finden sich Werte für Formel 25) S. 36 : 


$$
\eta(\mathrm{r})=-\frac{\mathrm{I}}{\pi^{2}} \cdot \frac{\mathrm{m}}{\mathrm{h}}\left[\frac{\operatorname{arctg}\left(\frac{\mathrm{h}}{\mathrm{a}}\right)}{\sqrt{\mathrm{r}^{2}-\mathrm{a}^{2}}}-\frac{\mathrm{I}}{\sqrt{\mathrm{r}^{2}+\mathrm{h}^{2}}} \cdot \operatorname{arccotg}\left(\frac{\mathrm{a}}{\mathrm{h}} \sqrt{\frac{\mathrm{r}^{2}+\mathrm{h}^{2}}{\mathrm{r}^{2}-\mathrm{a}^{2}}}\right)\right.
$$

und der Kraftlinienkonstruktion wegen für Formel 30), S. 37:

$$
\mathfrak{q}(\mathbf{r})=-\frac{2}{\pi} \cdot \mathbf{m}\left[\frac{\sqrt{\mathrm{r}^{2}-\mathrm{a}^{2}}}{\mathrm{~h}} \operatorname{arctg}\left(\frac{\mathrm{h}}{\mathrm{a}}\right)-\frac{\sqrt{\mathrm{r}^{2}+\mathrm{h}^{2}}}{\mathrm{~h}} \cdot \operatorname{arccotg}\left(\frac{\mathrm{a}}{\mathrm{h}} \sqrt{\frac{\mathrm{r}^{2}+\mathrm{h}^{2}}{\mathrm{r}^{2}-\mathrm{a}^{2}}}\right)+\arccos \left(\frac{\mathrm{a}}{\mathrm{r}}\right)\right] .
$$

Tabelle für $\eta(r)$ und $q(r),(a=I$,

$\mathrm{m}=\mathrm{I}$ und $\mathrm{h}=2)$ :

\begin{tabular}{c|c|c}
\hline $\mathbf{r}$ & $\eta(\mathbf{r})$ & $\mathfrak{q}(\mathbf{r})$ \\
\hline $\mathbf{1}, 000$ & $-\infty$ & 0 \\
$\mathbf{1}, \mathbf{1 2 5}$ & $-0, \mathbf{1 2 5 6 7}$ & $-0,21990$ \\
$\mathbf{1}, 250$ & $-0,08060$ & $-0,31230$ \\
$\mathbf{1}, 375$ & $-0,05993$ & $-0,38349$ \\
$\mathbf{1}, 500$ & $-0,04745$ & $-0,44352$ \\
$\mathbf{1}, 625$ & $-0,03894$ & $-0,5096 \mathbf{1}$ \\
$\mathbf{1}, 750$ & $-0,03274$ & $-0,54342$ \\
$\mathbf{1}, 875$ & $-0,02801$ & $-0,58654$ \\
2,000 & $-0,02430$ & $-0,62613$ \\
$\mathbf{2 , 1 2 5}$ & $-0,021 \mathbf{1} 3$ & $-0,65265$ \\
$\infty$ & 0 & $\mathbf{1}$
\end{tabular}
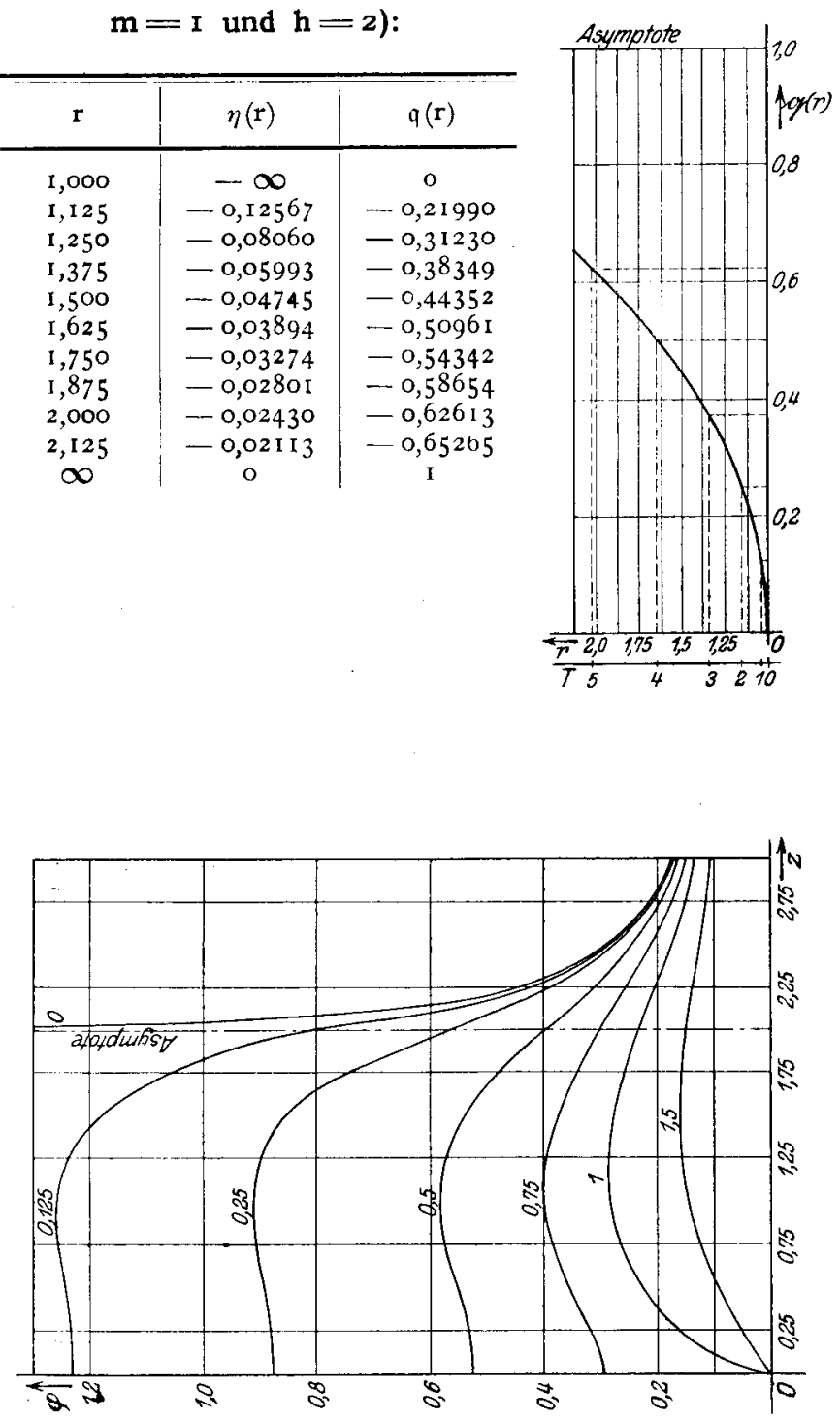
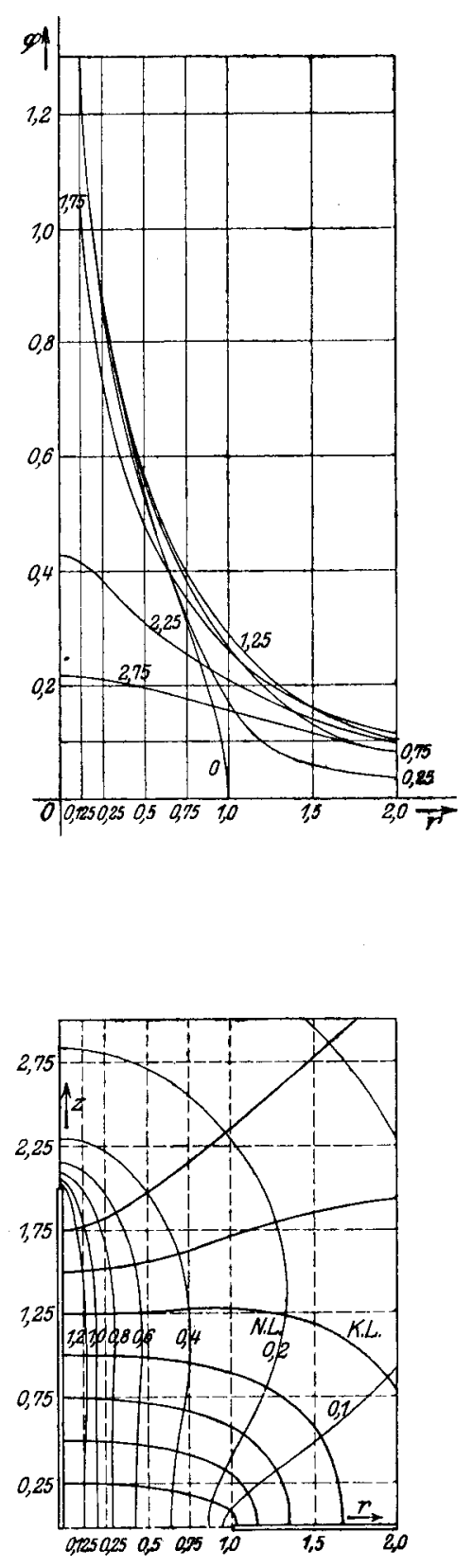
Zylinderhohlraumpotential. (Vergleiche Abschnitt IX.)

Formel I):

$$
\varphi_{1}=\frac{\mathrm{m}}{\sqrt{\mathrm{r}^{2}+\mathrm{z}^{2}}}
$$

Formel (3):

$$
\varphi_{2}=-\frac{\mathrm{m}}{\sqrt{\mathrm{R}^{2}+\mathrm{z}^{2}}}\left[\mathrm{I}-\frac{\left(\mathrm{R}^{2}-\mathrm{r}^{2}\right)\left(\mathrm{R}^{2}-2 \mathrm{z}^{2}\right)}{4\left(\mathrm{R}^{2}+\mathrm{z}^{2}\right)^{2}}\right]
$$

\begin{tabular}{|c|c|c|c|c|c|c|c|c|c|}
\hline \multirow{2}{*}{$z$} & \multirow{2}{*}{$\varphi$} & \multicolumn{8}{|c|}{$r$} \\
\hline & & 0 & $0, \mathrm{I}$ & 0,2 & 0,3 & 0,4 & 0,6 & 0,8 & 1,0 \\
\hline o & $\begin{array}{l}\varphi_{1} \\
-\varphi_{2} \\
\varphi\end{array}$ & $\begin{array}{l}+\infty \\
0,7500 \\
+\infty\end{array}$ & $\begin{array}{r}10,0000 \\
0,7525 \\
9,2475\end{array}$ & $\begin{array}{l}5,0000 \\
0,7600 \\
4,2400\end{array}$ & $\begin{array}{l}3,3333 \\
0,7725 \\
2,5608\end{array}$ & $\begin{array}{l}2,5000 \\
0,7900 \\
1,7100\end{array}$ & $\begin{array}{l}1,6667 \\
0,8400 \\
0,8267\end{array}$ & $\begin{array}{l}\mathbf{1}, 2500 \\
0,9100 \\
0,3400\end{array}$ & $\begin{array}{l}1,0000 \\
1,0000 \\
0,0000\end{array}$ \\
\hline $0, \mathrm{I}$ & $\begin{array}{r}\varphi_{1} \\
-\varphi_{3} \\
\varphi\end{array}$ & $\begin{array}{r}\text { I0,0000 } \\
0,756 \text { I } \\
0,2439\end{array}$ & $\begin{array}{l}7,0710 \\
0,7584 \\
6,3126\end{array}$ & $\begin{array}{l}4,4722 \\
0,7656 \\
3,7066\end{array}$ & $\begin{array}{l}3,1023 \\
0,7776 \\
2,3247\end{array}$ & $\begin{array}{l}2,4254 \\
0,7943 \\
1,63 \mathrm{II}\end{array}$ & $\begin{array}{l}1,6440 \\
0,8421 \\
0,8019\end{array}$ & $\begin{array}{l}1,2404 \\
0,9087 \\
0,3317\end{array}$ & $\begin{array}{c}0,995^{\circ} \\
0,995^{\circ} \\
0\end{array}$ \\
\hline 0,2 & $\begin{array}{c}\varphi_{1} \\
-\varphi_{2} \\
\varphi\end{array}$ & $\begin{array}{l}5,0000 \\
0,7721 \\
4,2279\end{array}$ & $\begin{array}{l}4,4722 \\
0,7741 \\
3,6981\end{array}$ & $\begin{array}{l}3,5356 \\
0,7804 \\
2,7552\end{array}$ & $\begin{array}{l}2,7735 \\
0,7908 \\
\mathrm{I}, 9827\end{array}$ & $\begin{array}{l}2,236 \mathrm{I} \\
0,8054 \\
\mathrm{I}, 4307\end{array}$ & $\begin{array}{l}\mathrm{I}, 58 \mathrm{I} 2 \\
0,847 \mathrm{I} \\
0,734 \mathrm{I}\end{array}$ & $\begin{array}{l}1,2 \text { I27 } \\
0,9055 \\
0,3072\end{array}$ & $\begin{array}{c}0,9806 \\
0,9806 \\
0\end{array}$ \\
\hline 0,3 & $\begin{array}{c}\varphi_{1} \\
-\varphi_{2} \\
\varphi\end{array}$ & $\begin{array}{l}3,3333 \\
0,7926 \\
2,5407\end{array}$ & $\begin{array}{l}3,1023 \\
0,7942 \\
2,3081\end{array}$ & $\begin{array}{l}2,7735 \\
0,7992 \\
1,9743\end{array}$ & $\begin{array}{l}2,3570 \\
0,8074 \\
\mathrm{I}, 5496\end{array}$ & $\begin{array}{l}2,0000 \\
0,8190 \\
1,1810\end{array}$ & $\begin{array}{l}\mathrm{I}, 4907 \\
0,8520 \\
0,6387\end{array}$ & $\begin{array}{l}\mathrm{I}, 1704 \\
0,8983 \\
0,2721\end{array}$ & $\begin{array}{c}0,9578 \\
0,9578 \\
0\end{array}$ \\
\hline 0,4 & $\begin{array}{c}\varphi_{1} \\
-\varphi_{2} \\
\varphi\end{array}$ & $\begin{array}{l}2,5000 \\
0,8112 \\
1,6888\end{array}$ & $\begin{array}{l}2,4254 \\
0,8 \text { I } 23 \\
1,6 \text { I } 31\end{array}$ & $\begin{array}{l}2,2361 \\
0,8159 \\
1,4202\end{array}$ & $\begin{array}{l}2,0000 \\
0,8217 \\
1,1783\end{array}$ & $\begin{array}{l}1,7677 \\
0,8299 \\
0,9378\end{array}$ & $\begin{array}{l}\mathbf{I}, 3867 \\
0,8534 \\
0,5333\end{array}$ & $\begin{array}{l}\text { I, I I } 8 \text { I } \\
0,8862 \\
0,23 \text { I } 9\end{array}$ & $\begin{array}{c}0,9285 \\
0,9285 \\
o\end{array}$ \\
\hline 0,6 & $\begin{array}{c}\varphi_{1} \\
-\varphi_{2} \\
\varphi\end{array}$ & $\begin{array}{l}\mathrm{I}, 6667 \\
0,8250 \\
0,8417\end{array}$ & $\begin{array}{l}1,6440 \\
0,8254 \\
0,8186\end{array}$ & $\begin{array}{l}\mathrm{r}, 58 \mathrm{I} 2 \\
0,8263 \\
0,7549\end{array}$ & $\begin{array}{l}\mathrm{I}, 4907 \\
0,8280 \\
0,6627\end{array}$ & $\begin{array}{l}\mathrm{I}, 3867 \\
0,8302 \\
0,5565\end{array}$ & $\begin{array}{l}\text { I, I } 788 \\
0,8367 \\
0,342 \text { I }\end{array}$ & $\begin{array}{l}\mathrm{I}, 0000 \\
0,8458 \\
0, \mathrm{I} 542\end{array}$ & $\begin{array}{c}0,8575 \\
0,8575 \\
0\end{array}$ \\
\hline 0,8 & $\begin{array}{r}\varphi_{1} \\
-\varphi_{2} \\
\varphi\end{array}$ & $\begin{array}{l}1,2500 \\
0,8012 \\
0,4488\end{array}$ & $\begin{array}{l}I, 2404 \\
0,8010 \\
0,4394\end{array}$ & $\begin{array}{l}\text { I , } 2 \text { I } 27 \\
0,8004 \\
0,4 \text { I } 23\end{array}$ & $\begin{array}{l}\mathrm{I}, \mathrm{I} 704 \\
0,7994 \\
0,3710\end{array}$ & $\begin{array}{l}\text { I, I I I I } \\
0,7979 \\
0,3202\end{array}$ & $\begin{array}{r}1,0000 \\
0,7939 \\
0,2061\end{array}$ & $\begin{array}{l}0,8839 \\
0,7882 \\
0,0957\end{array}$ & $\begin{array}{c}0,7809 \\
0,7809 \\
0\end{array}$ \\
\hline $\mathrm{I}, \mathrm{O}$ & $\begin{array}{c}\varphi_{1} \\
-\varphi_{2} \\
\varphi\end{array}$ & $\begin{array}{l}1,0000 \\
0,7513 \\
0,2487\end{array}$ & $\begin{array}{l}0,995^{\circ} \\
0,75^{\circ 8} \\
0,2442\end{array}$ & $\begin{array}{l}0,9806 \\
0,7495 \\
0,23 \text { I I }\end{array}$ & $\begin{array}{l}0,9578 \\
0,7473 \\
0,2105\end{array}$ & $\begin{array}{l}0,9285 \\
0,7442 \\
0,1843\end{array}$ & $\begin{array}{l}0,8575 \\
0,7354 \\
0,1221\end{array}$ & $\begin{array}{l}0,7809 \\
0,7230 \\
0,0579\end{array}$ & $\begin{array}{c}0,707 \mathrm{I} \\
0,707 \mathrm{I} \\
0\end{array}$ \\
\hline 1,2 & $\begin{array}{c}\varphi_{1} \\
-\varphi_{2} \\
\varphi\end{array}$ & $\begin{array}{l}0,8333 \\
0,6907 \\
0,1426\end{array}$ & $\begin{array}{l}0,8305 \\
0,6902 \\
0,1403\end{array}$ & $\begin{array}{l}0,8220 \\
0,6886 \\
0,1334\end{array}$ & $\begin{array}{l}0,8085 \\
0,6862 \\
0,1223\end{array}$ & $\begin{array}{l}0,7906 \\
0,6826 \\
0,1080\end{array}$ & $\begin{array}{l}0,7453 \\
0,6725 \\
0,0728\end{array}$ & $\begin{array}{l}0,6934 \\
0,6584 \\
0,035^{\circ}\end{array}$ & $\begin{array}{c}0,6402 \\
0,6402 \\
0\end{array}$ \\
\hline $\mathrm{I}, 4$ & $\begin{array}{r}\varphi_{1} \\
-\varphi_{2} \\
\varphi\end{array}$ & $\begin{array}{l}0,7143 \\
0,6597 \\
0,0546\end{array}$ & $\begin{array}{l}0,7 \text { I } 25 \\
0,6589 \\
0,0536\end{array}$ & $\begin{array}{l}0,7071 \\
0,6566 \\
0,0505\end{array}$ & $\begin{array}{l}0,6984 \\
0,6527 \\
0,0457\end{array}$ & $\begin{array}{l}0,6868 \\
0,6472 \\
0,0396\end{array}$ & $\begin{array}{l}0,6565 \\
0,63 \text { I } 5 \\
0,025^{\circ}\end{array}$ & $\begin{array}{l}0,6155 \\
0,6122 \\
0,0033\end{array}$ & $\begin{array}{c}0,58 \mathrm{I}_{2} \\
0,58 \mathrm{I} 2 \\
0\end{array}$ \\
\hline
\end{tabular}

$$
\varphi=\varphi_{1}+\varphi_{2} \text {. }
$$

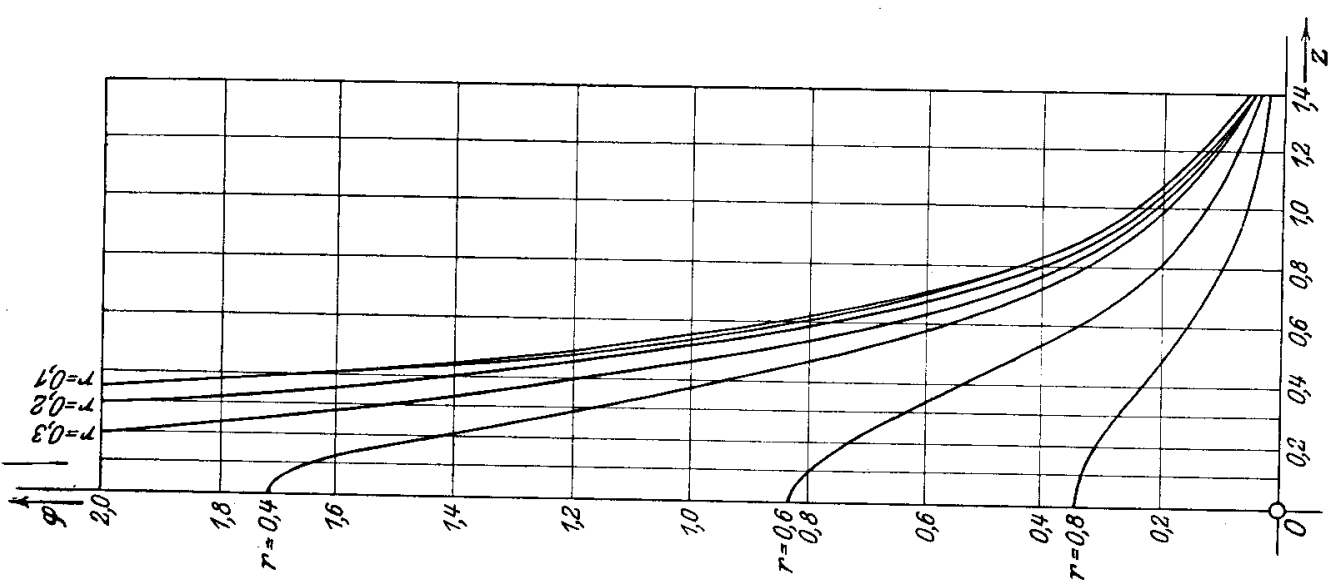



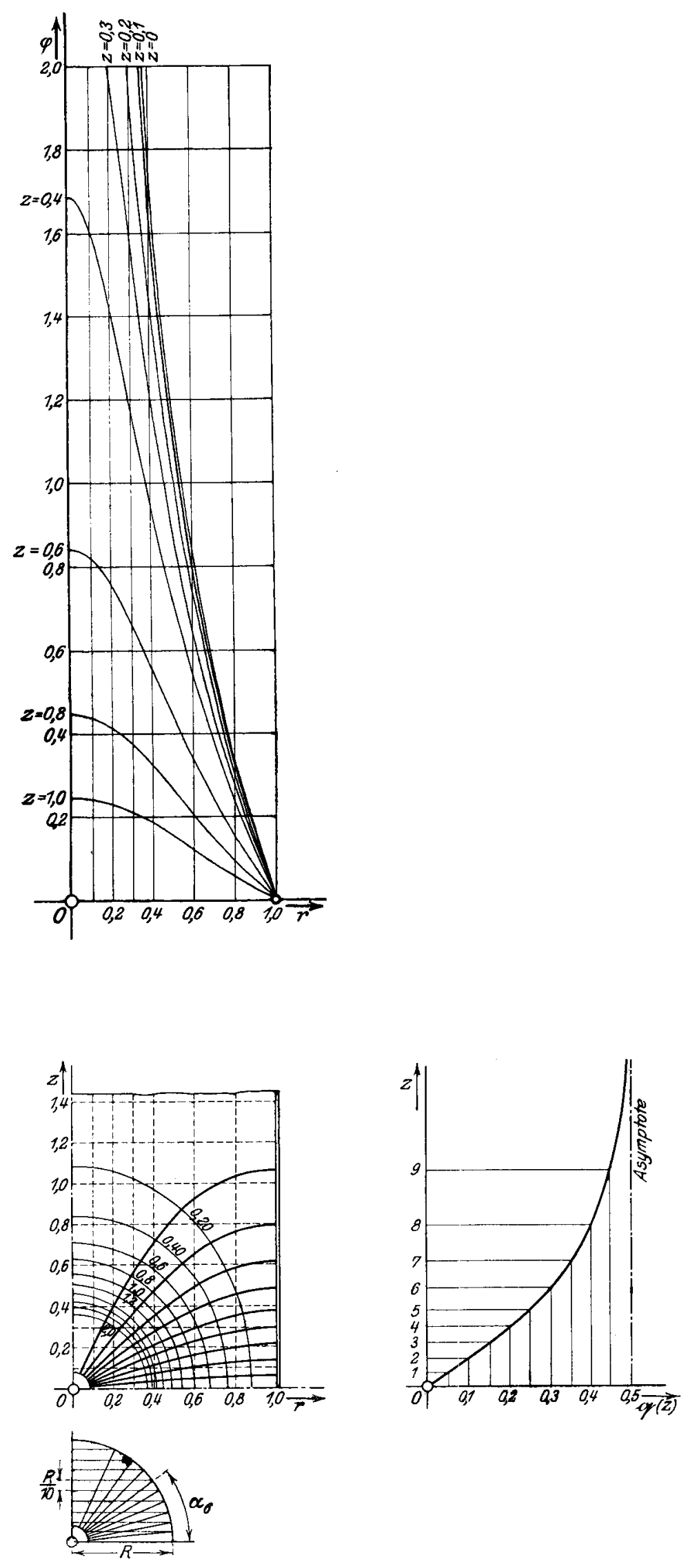


\section{Curriculum vitae.}

Am I4. März 1892 wurde ich in Basel geboren. Nach Besuch der dortigen Primarschule (I898-I902) und des untern Gymnasiums (I902-I905) trat ich im Frühjahr 1905 in die Zürcherische Kantonsschule ein, besuchte in dieser die zweite Gymnasialklasse und in den Jahren I906-rgro die Industrieschule (Oberrealschule), wo ich im Herbste I9Io die Maturitätsprüfung bestand. Während vier Jahren studierte ich hierauf an der eidgenössischen technischen Hochschule Ingenieurwissenschaften. Ich hörte Vorlesungen bei den Herren Dozenten:

Einstein, Escher, Farny, Geiser, Großmann, Hirsch, Kuhlmann, Meißner, Meyer, Schweitzer, Stodola, H. F. Weber, WeiB, Weyl, Wy $\mathrm{Bling}$.

Allen meinen verehrten Lehrern bin ich zu Dank verpflichtet. Insbesondere schulde ich Herrn Prof. Dr. K. Kuhlmanr, bei dem ich im Jahre r9I4 mein schriftliches Diplomexamen bestand, und Herrn Prof. Dr. M. Großmann, bei welchem ich im Winter I9I4/I5 als Assistent tätig war, für mancherlei Belehrungen und Förde. rungen großen Dank.

Unterdessen war ich Aushilfslehrer für die mathematischen Fächer an der Oberrealabteilung der Kantonsschule in Zürich und bin nun Assistent für theoretische Elektrotechnik und Elektromaschinenbau bei Herrn Prof. Dr. Kuhlmann bzw. dessen Stellvertreter während der Kriegszeit Herrn Prof. Dr. A. Schweitzer.

\section{Adolf Bolliger.}


Druck von Oscar Brandstetter in Leipzig. 